\title{
The three-dimensional substrate of atrial fibrillation in the goat : a simultaneous endo-epicardial mapping study
}

Citation for published version (APA):

Eckstein, J. (2012). The three-dimensional substrate of atrial fibrillation in the goat : a simultaneous endoepicardial mapping study. [Doctoral Thesis, Maastricht University]. Datawyse / Universitaire Pers Maastricht. https://doi.org/10.26481/dis.20120118je

Document status and date:

Published: 01/01/2012

DOI:

10.26481/dis.20120118je

Document Version:

Publisher's PDF, also known as Version of record

\section{Please check the document version of this publication:}

- A submitted manuscript is the version of the article upon submission and before peer-review. There can be important differences between the submitted version and the official published version of record.

People interested in the research are advised to contact the author for the final version of the publication, or visit the DOI to the publisher's website.

- The final author version and the galley proof are versions of the publication after peer review.

- The final published version features the final layout of the paper including the volume, issue and page numbers.

Link to publication

\footnotetext{
General rights rights.

- You may freely distribute the URL identifying the publication in the public portal. please follow below link for the End User Agreement:

www.umlib.nl/taverne-license

Take down policy

If you believe that this document breaches copyright please contact us at:

repository@maastrichtuniversity.nl

providing details and we will investigate your claim.
}

Copyright and moral rights for the publications made accessible in the public portal are retained by the authors and/or other copyright owners and it is a condition of accessing publications that users recognise and abide by the legal requirements associated with these

- Users may download and print one copy of any publication from the public portal for the purpose of private study or research.

- You may not further distribute the material or use it for any profit-making activity or commercial gain

If the publication is distributed under the terms of Article $25 \mathrm{fa}$ of the Dutch Copyright Act, indicated by the "Taverne" license above, 


\title{
The Three-dimensional Substrate of Atrial Fibrillation in the Goat
}

\author{
A Simultaneous Endo-epicardial \\ Mapping Study
}

Jens Eckstein 
(C) Copyright Jens Eckstein, Maastricht 2012 ISBN 9789461590978

Production: Datawyse / Universitaire Pers Maastricht 


\section{The Three-dimensional Substrate of Atrial Fibrillation in the Goat}

\section{A Simultaneous Endo-epicardial Mapping Study}

\section{Proefschrift}

ter verkrijging van de graad van doctor

aan de Universiteit Maastricht, op gezag van de Rector Magnificus,

Prof. mr. G.P.M.F. Mols,

volgens het besluit van de College van Decanen, in het openbaar te verdedigen

op woensdag 18 januari 2012 om 16:00 uur

door

\section{Jens Eckstein}

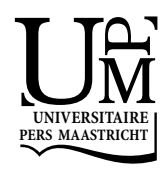


Promotores

Beoordelingscommissie
Prof. dr. Maurits A. Allessie

Prof. dr. Ulrich Schotten

Prof. dr. Harry J.G.M. Crijns (Voorzitter)

Prof. dr. Jacques M.T. de Bakker

Prof. dr. Frits W. Prinzen

Prof. dr. Isabelle Van Gelder

The studies of this thesis were supported by grants of the Swiss National Foundation, the Marie Curie Program and the Fondation Leducq. 
Chapter 2: Mechanisms of Perpetuation of Atrial Fibrillation in Chronically Dilated Atria

Chapter 3: Time Course and Mechanisms of Endo-epicardial Electrical Dissociation during Atrial Fibrillation in the Goat

Chapter 4: Correlation between Endo-epicardial Dissociation And Transmural Conduction during Acute and Persistent Atrial Fibrillation in the Goat

Chapter 5: Effects of Flecainide on Endo-Epicardial Dissociation And Incidence of Breakthrough

Chapter 6: Mapping of Atrial Fibrillation - 



\section{Chapter 1}

\section{Introduction}

Clinical aspects and therapeutic options

Remodeling and stabilization of AF

Aim of the study 


\section{Introduction}

Normal, regular heart rhythm is driven by the specific electrophysiological properties of the sinus nodal cells. For various reasons, atrial fibrillation (AF) occurs in approximately $1-2 \%$ of the general population with an increasing prevalence up to $15 \%$ in elderly people, making AF the most common human arrhythmia. ${ }^{1,2}$ During atrial fibrillation the atria are not activated in a coordinated regular fashion like during sinus rhythm, but in a chaotic activation pattern resulting in fibrillating rather than contracting atria. This results in a loss of pump function, an irregular, often too rapid ventricular rhythm, and an adaptation process referred to as atrial remodeling. Diagnosis usually is confirmed by a surface electrocardiogram (ECG) meeting the following three criteria:

(1) 'Absolutely' irregular RR intervals

(2) No distinct $P$ waves on the surface ECG

(3) Atrial cycle length (if detectable) usually variable and approximately $200 \mathrm{~ms}$ (figure 1). ${ }^{3}$
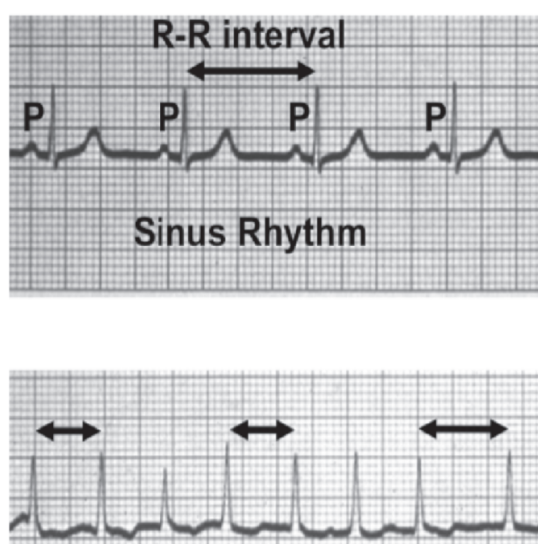

Atrial Fibrillation
Figure 1: Surface ECG tracings of sinus rhythm and $\operatorname{AF}(25 \mathrm{~mm} / \mathrm{s}, 10 \mathrm{~mm}=1 \mathrm{mV})$.

Upper tracing shows sinus rhythm with evident $p$ waves (marked with "P") and regular RRintervals (double arrows).

Lower tracing shows atrial fibrillation with missing p-waves, absolutely irregular RR-intervals, and rapid ventricular rhythm (tachyarrhythmia). 


\section{Clinical aspects and therapeutic options}

AF is related to an increased morbidity and mortality if untreated. ${ }^{1,4}$ Symptoms of AF range from asymptomatic ("silent AF") to severe heart failure due to tachymyopathy. ${ }^{4}$ Cardioembolic cerebrovascular stroke is the most relevant AF-related complication. AF patients have a five fold increased stroke risk and every fifth stroke is related to $A F .^{4,5}$ The incidence of stroke is not related to symptoms or type of AF (paroxysmal or persistent) but strongly depends on the concomitant comorbidities of the patients. To calculate the individual stroke risk, the $\mathrm{CHA}_{2} \mathrm{DS}_{2} \mathrm{VASc}$ score was introduced in clinical practice. ${ }^{6,7}$ The stroke risk should then be weighed against the risk of bleeding which can be estimated with the use of the HAS-BLED score and a recommendation for or against oral anticoagulation should be given to the patient. ${ }^{8}$

Patients presenting with AF in daily clinical practice are an inhomogeneous population and most likely have different triggers und mechanisms maintaining AF. Besides known and common risk factors for AF like hypertension, valvular heart disease and age, specific circumstances like hyperthyroidism, cardiac surgery or acute infections can trigger episodes of $\mathrm{AF}^{9,}{ }^{90}$ In such patients the mechanisms underlying and triggering the arrhythmia may be different and can require different treatment strategies.

Rhythm control (restoration of sinus rhythm) and rate control (pharmacological control of the ventricular rate) are equally effective therapies in respect to mortality and stroke..$^{3,11,12}$ Thus, rate control is a therapeutic option to be considered at any time. 
The appropriate therapeutic approach should therefore primarily be aimed at the symptoms of the patient and complications associated with AF.

If a rhythm control strategy is chosen, therapeutic options to restore sinus rhythm are pharmacological (flecainide, propafenone, amiodarone, ibutilide or vernakalant) or electrical cardioversion (direct current cardioversion). In most patients an ongoing antiarrhythmic drug therapy will be needed to maintain sinus rhythm. If drug therapy fails to maintain sinus rhythm or has to be terminated because of side effects, catheter-, or surgical ablation offer an invasive therapeutic alternative. According to the present ESC guidelines catheter ablation can be chosen as first line therapy in patients with no or only minimal heart disease and paroxysmal AF.

If a rate control strategy is chosen, pharmacological rate control is the first line therapy ( $\beta$-blockers, non-dihydropyridine calcium antagonists, digitalis) and if it fails AV-node ablation combined with implantation of a biventricular pacemaker offers an alternative therapy to improve the symptoms of AF patients.

Once AF evolves, multiple physiological responses stabilize the arrhythmia over time and finally make it resistant to cardioversion and/or maintenance of sinus rhythm. This is why an understanding of these mechanisms is crucial for a focused and successful treatment strategy. 


\section{Remodeling and stabilization of AF}

In most patients the "natural course" of AF starts with paroxysmal self-limiting AF episodes (paroxysmal AF). Without successful treatment, the AF episodes will eventually become sustained and AF termination will require electrical and/or pharmacological cardioversion (persistent AF). Again, if treatment fails to maintain sinus rhythm, AF often cannot be successfully cardioverted anymore (long-standing persistent AF). When, in long-standing persistent AF, the therapeutic option of rhythm control is rejected and a rate control strategy is chosen the recommended clinical term is "permanent AF".

While electrical remodeling with shortening of the atrial refractory period during the first days of $A F$ represents a well-studied explanation for the initial stabilization of $A F$ within hours to days, ${ }^{3,13}$ mechanisms contributing to the increasing stabilization of AF over months and years are still a matter of ongoing scientific research and debate. $^{10,} 14$ A point of major interest in the discussion about mechanisms contributing to the stabilization of $\mathrm{AF}$ is the generation of new fibrillation waves. In this context, fibrillation waves that seem to originate from a spot/focus within the mapping area (in contrast to waves entering the mapping area from the side and propagating through it) have attracted attention by several researcher groups. These waves were described as "breakthrough waves" earlier, according to the hypothesis that they originate from transmurally conducted endocardial fibrillation waves that "break through" to the epicardial layer and form a new wave front there. ${ }^{15}$ Indirect evidence supporting the breakthrough hypothesis was described in patients and animal models. ${ }^{15-17}$ In a recent study, De Groot showed unipolar electrogram morphology presenting an R/S-constellation at the sites of breakthrough, highly 
variable non-repetitive sites of breakthrough and a coupling interval not more than $11 \mathrm{~ms}$ shorter than the average AF cycle length in patients with AF. However, no in vivo simultaneous endo-epicardial high-resolution contact mapping was available so far to demonstrate the contralateral electrical activity at a breakthrough site.

An alternative origin of breakthrough waves could be ectopic focal discharges triggered by delayed afterdepolarizations. Evidence supporting this hypothesis derives from human and animal experiments. Neef found an increased diastolic sarcoplasmatic reticulum calcium leak in AF patients, which leads to an increased intracellular calcium load and can trigger delayed afterdepolarisations. ${ }^{18}$

Indirect evidence was found by Knollmann and coworkers who demonstrated that the Class $1 \mathrm{C}$ drug flecainide specifically blocks the ryanodine receptor $\mathrm{Ca}^{2}$ release channels in the open state leading to a decreased incidence of sarcoplasmic $\mathrm{Ca}^{2}$ release events ("sparks"). ${ }^{19-21}$ This leads to a reduced incidence of intracellular $\mathrm{Ca}$ waves and a decreased incidence of delayed afterdepolarisations. Also here, simultaneous endo- epicardial mapping studies would be required to further investigate the role of ectopic focal discharges in the genesis of breakthrough waves. 


\section{Aim of the study}

Aim of the present study was

1. To identify differences and similarities in the electrophysiological behavior between the endocardial bundle network and the epicardial layer.

2. To investigate whether endo-epicardial dissociation of electrical activity is a conditio sine qua non for the occurrence of breakthrough.

3. To clarify whether breakthrough events originate from transmural activation by fibrillation waves propagating in the contralateral layer of the atrial wall or from ectopic focal discharges.

4. To study the effect of flecainide on endo- and epicardial wave characteristics, endo-epicardial dissociation and the incidence of breakthroughs. 


\section{References}

1. Stewart S, Hart CL, Hole DJ, McMurray JJ. Population prevalence, incidence, and predictors of atrial fibrillation in the Renfrew/Paisley study. Heart. 2001;86(5):516-521.

2. Miyasaka Y, Barnes ME, Gersh BJ, Cha SS, Bailey KR, Abhayaratna WP, Seward JB, Tsang TS. Secular trends in incidence of atrial fibrillation in Olmsted County, Minnesota, 1980 to 2000, and implications on the projections for future prevalence. Circulation. 2006;114(2):119-125.

3. Camm AJ, Kirchhof P, Lip GY, Schotten U, Savelieva I, Ernst S, Van Gelder IC, Al-Attar N, Hindricks G, Prendergast B, Heidbuchel H, Alfieri O, Angelini A, Atar D, Colonna P, De Caterina R, De Sutter J, Goette A, Gorenek B, Heldal M, Hohloser SH, Kolh P, Le Heuzey JY, Ponikowski P, Rutten FH. Guidelines for the management of atrial fibrillation: the Task Force for the Management of Atrial Fibrillation of the European Society of Cardiology (ESC). Eur Heart J. 2010;31(19):2369-2429.

4. Kirchhof $P$, Auricchio A, Bax J, Crijns $H$, Camm J, Diener HC, Goette A, Hindricks G, Hohnloser S, Kappenberger L, Kuck KH, Lip GY, Olsson B, Meinertz T, Priori S, Ravens U, Steinbeck G, Svernhage E, Tijssen J, Vincent A, Breithardt G. Outcome parameters for trials in atrial fibrillation: recommendations from a consensus conference organized by the German Atrial Fibrillation Competence NETwork and the European Heart Rhythm Association. Europace. 2007;9(11):1006-1023.

5. Friberg L, Hammar N, Rosenqvist M. Stroke in paroxysmal atrial fibrillation: report from the Stockholm Cohort of Atrial Fibrillation. Eur Heart J. 2010;31(8):967-975.

6. Lip GY, Frison L, Halperin JL, Lane DA. Identifying patients at high risk for stroke despite anticoagulation: a comparison of contemporary stroke risk stratification schemes in an anticoagulated atrial fibrillation cohort. Stroke. 2010;41(12):2731-2738.

7. Lip GY, Nieuwlaat R, Pisters R, Lane DA, Crijns HJ. Refining clinical risk stratification for predicting stroke and thromboembolism in atrial fibrillation using a novel risk factor-based approach: the euro heart survey on atrial fibrillation. Chest. 2010;137(2):263-272.

8. Pisters R, Lane DA, Nieuwlaat R, de Vos CB, Crijns HJ, Lip GY. A novel userfriendly score (HAS-BLED) to assess 1-year risk of major bleeding in patients with atrial fibrillation: the Euro Heart Survey. Chest. 2010;138(5):1093-1100. 
9. Maesen B, Nijs J, Maessen J, Allessie M, Schotten U. Post-operative atrial fibrillation: a maze of mechanisms. Europace. 2011.

10. Schotten U, Verheule S, Kirchhof P, Goette A. Pathophysiological mechanisms of atrial fibrillation: a translational appraisal. Physiol Rev. 2011;91(1):265-325.

11. Van Gelder IC, Hagens VE, Bosker HA, Kingma JH, Kamp O, Kingma T, Said SA, Darmanata JI, Timmermans AJ, Tijssen JG, Crijns HJ. A comparison of rate control and rhythm control in patients with recurrent persistent atrial fibrillation. N Engl J Med. 2002;347(23):1834-1840.

12. Wyse DG, Waldo AL, DiMarco JP, Domanski MJ, Rosenberg $Y$, Schron EB, Kellen JC, Greene HL, Mickel MC, Dalquist JE, Corley SD. A comparison of rate control and rhythm control in patients with atrial fibrillation. $N$ Engl J Med. 2002;347(23):1825-1833.

13. Wijffels MC, Kirchhof CJ, Dorland R, Allessie MA. Atrial fibrillation begets atrial fibrillation. A study in awake chronically instrumented goats. Circulation. 1995;92(7):1954-1968.

14. Eckstein J, Verheule S, de Groot N, Allessie M, Schotten U. Mechanisms of perpetuation of atrial fibrillation in chronically dilated atria. Prog Biophys Mol Biol. 2008;97(2-3):435-451.

15. Konings KT, Kirchhof CJ, Smeets JR, Wellens HJ, Penn OC, Allessie MA. High-density mapping of electrically induced atrial fibrillation in humans. Circulation. 1994;89(4):1665-1680.

16. Holm M, Johansson R, Brandt J, Luhrs C, Olsson SB. Epicardial right atrial free wall mapping in chronic atrial fibrillation. Documentation of repetitive activation with a focal spread--a hitherto unrecognised phenomenon in man. Eur Heart J. 1997;18(2):290-310.

17. de Groot NM, Houben RP, Smeets JL, Boersma E, Schotten U, Schalij MJ, Crijns $\mathrm{H}$, Allessie MA. Electropathological substrate of longstanding persistent atrial fibrillation in patients with structural heart disease: epicardial breakthrough. Circulation. 2010;122(17):1674-1682.

18. Neef S, Dybkova N, Sossalla S, Ort KR, Fluschnik N, Neumann K, Seipelt R, Schondube FA, Hasenfuss G, Maier LS. CaMKII-dependent diastolic SR $\mathrm{Ca} 2+$ leak and elevated diastolic $\mathrm{Ca} 2+$ levels in right atrial myocardium of patients with atrial fibrillation. Circ Res. 2010;106(6):1134-1144.

19. Hwang HS, Hasdemir C, Laver D, Mehra D, Turhan K, Faggioni M, Yin H, Knollmann BC. Inhibition of Cardiac Ca2+ Release Channels (RyR2) Determines Efficacy of Class I Antiarrhythmic Drugs in Catecholaminergic Polymorphic Ventricular Tachycardia. Circ Arrhythm Electrophysiol. 2011. 
20. Hilliard FA, Steele DS, Laver D, Yang Z, Le Marchand SJ, Chopra N, Piston DW, Huke S, Knollmann BC. Flecainide inhibits arrhythmogenic $\mathrm{Ca} 2+$ waves by open state block of ryanodine receptor $\mathrm{Ca} 2+$ release channels and reduction of Ca2+ spark mass. J Mol Cell Cardiol. 2010;48(2):293-301.

21. Watanabe H, Chopra N, Laver D, Hwang HS, Davies SS, Roach DE, Duff HJ, Roden DM, Wilde AA, Knollmann BC. Flecainide prevents catecholaminergic polymorphic ventricular tachycardia in mice and humans. Nat Med. 2009;15(4):380-383. 


\title{
Chapter 2
}

\section{Mechanisms of Perpetuation of}

\author{
Atrial Fibrillation
}

\section{in Chronically Dilated Atria}

Jens Eckstein, $\mathrm{MD}^{1}$, Sander Verheule, $\mathrm{PhD}^{1}$, Natasja de Groot, $\mathrm{MD}, \mathrm{PhD}^{2}$, Maurits Allessie, MD, $\mathrm{PhD}^{1}$, Ulrich Schotten, $\mathrm{MD}, \mathrm{PhD}^{1}$

1: Cardiovascular Research Institute, Department of Physiology, University Maastricht, Maastricht, The Netherlands,

2: Department of Cardiology, Leiden University Medical Center, Leiden, The Netherlands

Published in: Prog Biophys Mol Biol. Jun-Jul 2008;97(2-3):435-451. 


\section{Abstract}

The progressive nature of atrial fibrillation (AF) has been demonstrated in numerous experimental as well as clinical investigations. Electrical remodeling (shortening of atrial refractoriness) develops within the first days of AF and contributes to the increase in stability of the arrhythmia. However, 'domestication of AF' must also depend on other mechanisms since the stability of AF continues to increase after electrical remodeling has been completed. Chronic atrial stretch induces activation of numerous signaling pathways leading to cellular hypertrophy, fibroblast proliferation and tissue fibrosis. The resulting electro-anatomical substrate is characterized by increased non-uniform anisotropy and local conduction heterogeneities facilitating reentry in the dilated atria. Atrial fibrosis may lead to disruption of the electrical sideto-side junctions between muscle bundles. This can result in electrical dissociation between neighboring muscle bundles, i.e. they become activated out-of-phase. Recent mapping studies in goats with persistent AF showed that electrical dissociation can not only occur between neighboring muscle bundles but also in the 3rd dimension, i.e. between the epicardial layer and the endocardial bundle network. Such endo-epicardial dissociation will significantly increase the number of wavefronts which can simultaneously be present in the atrial wall. This article reviews data suggesting a role of endo-epicardial dissociation in dilated and fibrillating atria, for the self-perpetuating nature of $\mathrm{AF}$ as well as its possible implications for therapeutic interventions. 


\section{Introduction}

After decades of basic and clinical research, the mechanisms of initiation and perpetuation of atrial fibrillation (AF) are still not fully understood. A large variety of mechanisms has been proposed, generally differing in the "sources" that are thought to be responsible for the arrhythmia. While some are based on the idea that local automaticity or triggered activity would drive AF, others describe different types of continuous conduction like reentry circuits or multiple wavelets as the key mechanism. Also, the combination of several mechanisms has been proposed to contribute to initiation and perpetuation of AF (Figure 1).

Reentry circuits have first been described in 1906 by Mayer in jelly fish and have been further implemented in cardiac electrophysiology by Mines studying a variety of animal hearts ${ }^{1,2}$. Since then reentry circuits have been shown to be the key mechanism in many arrhythmias such as atrial flutter, AV-node reentry tachycardia, and various forms of ventricular tachycardia. In 1959, the multiple wavelet theory was introduced by Moe and coworkers ${ }^{3}$. According to this hypothesis, multiple wavelets propagate through the atrium to wherever they encounter excitable tissue. In contrast to most other theories, this model does not require a certain source of rapid fibrillation waves, but proposes a balanced process of wavefront generation and extinction throughout the atrium (Figure 1 cartoon 7). In 1985, the first mapping studies of $\mathrm{AF}$ in isolated canine atria revealed a conduction pattern consistent with the presence of multiple wavefronts ${ }^{4}$. Since then, reentrant mechanisms of AF have been reported in a variety of animal models. For example, Kumagai et al demonstrated that multiple unstable reentrant circuits were responsible for maintenance of $\mathrm{AF}^{5}$ in a canine model of sterile pericarditis (Figure 1 Cartoon 6). In 
this model, multiple reentry circuits transiently followed anatomic pathways. Although each of the reentry circuits was not stable in it itself, together they sustained stable AF and constantly reactivated each other. A similar mechanism of reentry maintaining fibrillatory conduction in the periphery is effective if there is a stable reentrant mother wave maintaining the fibrillatory process in the periphery. A model of continuous activation from a single atrial source was described by Mandapati and Jalife ${ }^{6,7}$. In their experimental studies using a model of isolated sheep hearts exposed to high dosages of acetylcholine they observed stable and rapidly rotating activation patterns in the left atrium, driving fibrillatory conduction in the rest of the atria. Such a "rotor" can be anchored due to structural discontinuities, or it can move to a certain extend, still keeping its stable pattern and serving as a source of wave front generation. (Figure 1, Cartoon 3\&4). This observation formed the basis for a new strategy to identify sites with the highest dominant frequency as possible focal sources of AF and targets for ablation therapy. This approach was supported by the notion of a gradient of dominant frequencies in the atria of patients with paroxysmal AF by Lazar et al. ${ }^{8,9}$. Ablation of such sites indeed resulted in prolongation of the AF cycle length and finally cardioversion. However, frequency gradients could not consistently be demonstrated in all patients ${ }^{9,10}$. In patients with persistent $A F$, Sanders and colleagues demonstrated that ablation of sites with a higher dominant frequency than in adjacent areas did result in prolongation of AF cycle length, but not in termination of $\mathrm{AF}^{11}$.

All the above described models include continuously conducted excitation as the source of activation. Other experiments suggested that ectopic activity alone could sustain AF. The first model of local automaticity triggering arrhythmias goes back to 
experiments of Scherf who in 1949 applied aconitine to atria of dogs and thereby induced $\mathrm{AF}^{12}$. The exact mechanism remained unclear, but besides the postulated local automaticity, micro-reentry could not be ruled out because of the extreme shortening of the refractory period by aconitine (figure 1 cartoon 1 ).

\section{Single Source}

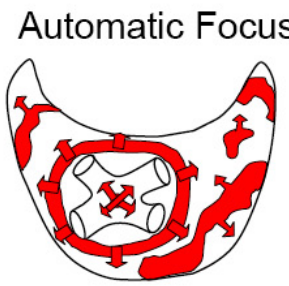

1

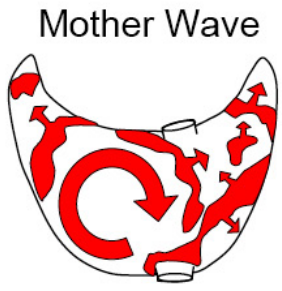

2

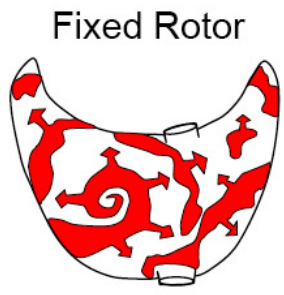

3
Moving Rotor

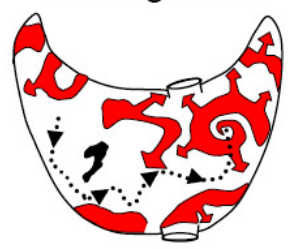

4

\section{Multiple Sources}

Focus

$+$
Multiple Foci Unstable Circuits

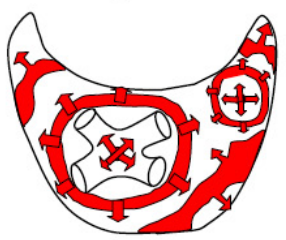

5

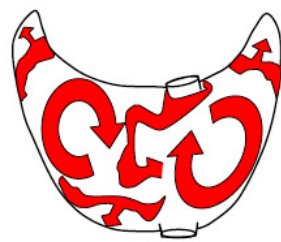

6

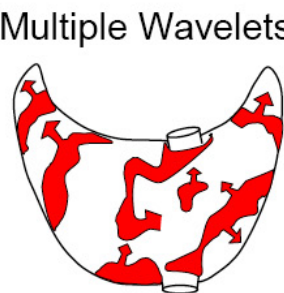

7

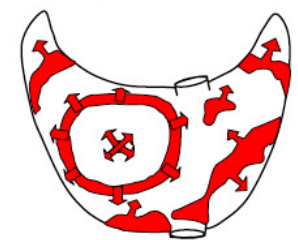

8

Figure 1: Cartoons of left (1 \& 5) and right atria demonstrating potential mechanisms of Atrial Fibrillation. Fibrillation waves are displayed in red with arrows indicating the direction of propagation.

Haissaguerre's work in 1998 introduced the concept of 'focal activity' as a trigger of $\mathrm{AF}^{13}$. In a group of 45 patients with paroxysmal AF they could demonstrate that the paroxysms were induced by focal activity ("bursts") mainly originating from the 
myocardial sleeves in the pulmonary veins (PVs). His work led to the now generally accepted view that episodes of paroxysmal AF are often triggered by bursts of local depolarizations originating from the PVs. The mechanisms underlying this "PVfiring", however, are still unknown. The local nature of these depolarizations is suggestive for automaticity in the PVs as the source of the fibrillatory process. In theory, however, the arrhythmia could also be triggered by small localized reentry circuits. Recently, the group of Berenfeld et al. addressed this question in a clinical study in patients with paroxysmal and persistent AF. They found that both the cycle length of dissociated PV depolarizations and the AF cycle length at sites of highest dominant frequency shortened after application of adenosine. Adenosine-induced activation of inward rectifier currents shortens the atrial action potential and stabilizes the resting membrane potential so that automaticity and triggered activity are inhibited while reentry circuits become more stable. Thus, their results strongly suggest a reentry mechanism underlying these dissociated PV arrhythmias ${ }^{14}$. On the other hand, Patterson et al. demonstrated that bursts from the PVs in patients often are initiated by atrial tachycardia followed by a pause. Endocardial mapping of the PVs revealed focal spread of activation of PV firing. The authors concluded that this spontaneous activity is most likely triggered by an elevated cytosolic $\mathrm{Ca}^{2+}$ after abbreviated action potentials causing delayed after-depolarizations (DADs) ${ }^{15}$. In summary mother waves, unstable circuits, rotors and multiple wavelets are based on mechanisms involving continuously conducted activity whereas PV foci are supposed to be due to local automaticity. The difference between the first four AF mechanisms consists of their spatial and/or temporal stability (moving vs. fixed, unstable vs. stable) and their activation pattern (rotor vs. circuit). 
Besides the different possible mechanisms leading to the initiation of $A F$, also adaptation processes of the atria (remodeling) influence the electrophysiological phenotype of the arrhythmia. An important contribution for the understanding of the changes occurring in the presence of AF came from work of Wijfels et al in $1995^{16}$. They demonstrated that AF itself induces a shortening of the atrial refractory period within a couple of days enabling more wavelets to coexist at the same time. They referred to this process as "electrical remodeling". This finding could, however, not explain why AF got increasingly stable over months and at a later point in time could not be cardioverted any more. Therefore, additional mechanisms explaining the progressive nature of AF have been postulated (the 'second factor' 17, 18. Chronic stretch and atrial dilatation appear to be important stimuli for chronic atrial structural remodeling which has been shown to largely facilitate induction and perpetuation of AF. The first description of structural remodeling by Ausma et al. was based on observations in a goat model of long-term AF (20weeks). During persistent AF, they found progressive hypertrophy of atrial myocytes associated with perinuclear glycogen accumulation, sarcomere depletion and myolyis ${ }^{19}$. However, the most extensive structural remodeling of the atrial myocardium has been described in chronically dilated atria. Although in most patient populations it can not be determined to what extent dilatation is a cause or consequence of $\mathrm{AF}$ an important role of atrial dilatation in the promotion of $A F$ is suggested by

1. epidemiological data demonstrating that atrial dilatation is an independent risk fact of $\mathrm{AF}$,

2. the fact that the AF inducibility and stability is increased in a large variety of animal models with atrial dilatation, and 
3. the observation that stretch activates intracellular signaling cascades which are known to produce structural remodeling in the atria ${ }^{20}$.

\section{Role of atrial dilatation in creating a substrate for AF}

Atrial dilatation can be both a cause and consequence of AF. Fraser and Turner already reported in 1955 that atrial enlargement correlated with the incidence of AF in patients with mitral valve disease ${ }^{21}$. In patients with valvular disease, Henry et al showed that $A F$ was rare $(3 \%)$ when the left atrial diameter was less than $40 \mathrm{~mm}$, but common (54\%) when it exceeded $40 \mathrm{~mm}{ }^{22}$. The Framingham Study has established left atrial size as a strong independent predictor for the development of $A F{ }^{23}, 24$. On the other hand, several studies imply that $A F$ also causes atrial enlargement. Keren et al. have reported that patients with mitral stenosis and SR had increased left atrial size but normal right atrial dimensions. However, both atria were enlarged in patients with mitral stenosis and AF and in lone AF patients ${ }^{25}$. In a small prospective echocardiographic study on patients with AF, a normal atrial size at baseline and no evidence of other cardiac abnormalities, the size of both atria increased during a 20 months follow-up period ${ }^{26}$. The investigators of the Stroke Prevention in Atrial Fibrillation (SPAF) trial have estimated that the independent contribution of chronic AF to the increase in left atrial diameter was about $2.5 \mathrm{~mm}^{27}$. Finally, cardioversion of AF has been shown to lead to a decrease in atrial dimensions ${ }^{28-30}$.

The effect of atrial dilatation on the substrate of AF has been studied in several animal models of acute and chronic atrial stretch. Data on effects of acute dilatation on atrial electrophysiology are diverging. In Langendorff-perfused rabbit hearts, 
increased atrial pressure caused a decrease in atrial effective refractory period (AERP) and action potential duration (APD) and an increase in AF inducibility ${ }^{31}$. These changes disappeared within 3 minutes upon release of atrial stretch. The electrophysiological effects of acute atrial stretch in rabbit hearts could be reduced by infusion of gadolinium, suggesting that stretch activated ion channels are responsible for the decrease in AERP and increase in AF vulnerability ${ }^{32}$. In dogs in which acute LA dilatation was produced by inflation of a balloon catheter, a shortening of the AERP and concomitant increase in susceptibility to atrial arrhythmias was found ${ }^{33}$. However, a prolongation of the AERP was measured in dogs subjected to acute RA stretch by saline infusion, ${ }^{34}$ whereas acute atrial dilatation by rapid infusion of a blood-expanding fluid in goats did not change the AERP ${ }^{35}$. The mechanism by which acute dilatation increases the AF vulnerability was studied in detail in rabbit atria using a mapping array of 240 electrodes on the right atrial wall ${ }^{36,37}$. In these experiments, acute dilatation caused increased local conduction slowing and block in the atrial free walls. Lines of block were preferentially oriented in the direction of the pectinate muscles (figure 2), suggesting that the thin epicardial layer overlaying this trabecular network is relatively sensitive to acute stretch. The electrophysiological effects of acute dilatation may also differ between atrial regions. Kalifa et al. showed that during AF episodes in perfused sheep atria, acute dilatation increased activation frequencies in the PVs, but not in the left atrial free walls ${ }^{38}$. 

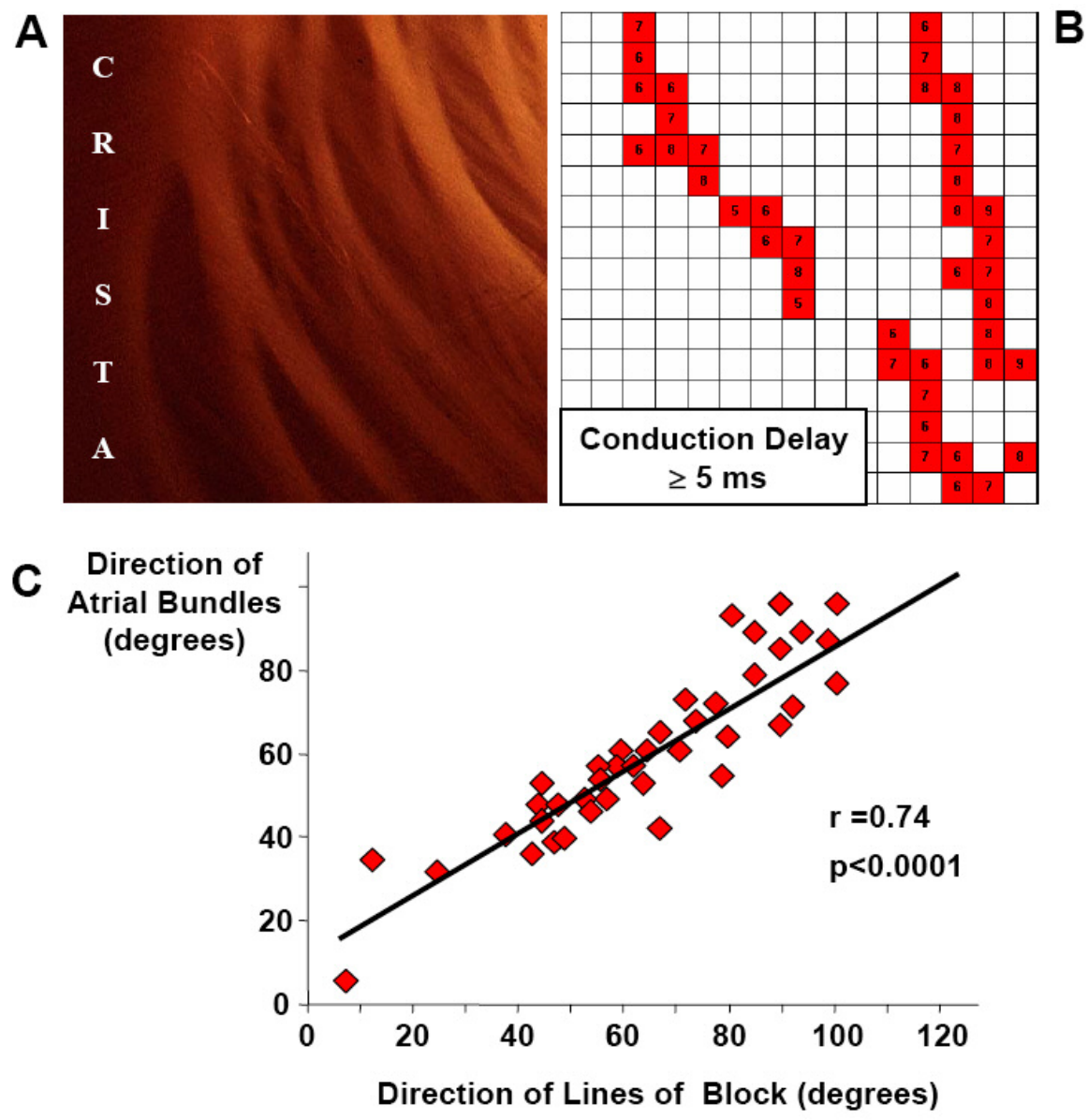

Figure 2: Correlation between lines of block and bundle architecture in rabbit atria during acute dilatation. A) Orientation of the right atrial endocardial bundle network extending from the crista terminalis to the AV-ring in rabbit atria dilated by the application of a hydrostatic pressure of $9 \mathrm{~cm} \mathrm{H} \mathrm{H}_{2} \mathrm{O}$. B) In the presence of the class I

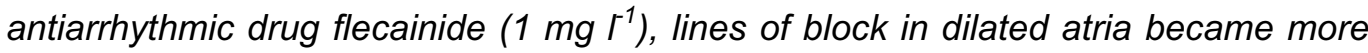
pronounced. These lines of block were oriented in parallel to the main bundle orientation.

C) Highly significant correlation between the orientation of lines of conduction block during pacing with the orientation of atrial bundles (from reference ${ }^{37}$ ). 
Chronic atrial dilatation occurs in several animal models with an increased AF vulnerability. In dogs with 'lone $\mathrm{AF}^{\prime}$ due to rapid atrial pacing with a controlled ventricular rate, left atrial area increased by $24 \%$ (estimated by considering the atrium as a sphere with an area of $\left.4 \pi r^{2}\right)^{39}$. This demonstrates that, as in patients, chronic AF leads to atrial dilatation. An increase in atrial size was also reported for several models of underlying heart disease with an increased AF vulnerability. In a canine model of congestive heart failure due to rapid ventricular pacing, the left atrial area increased by an estimated $80 \%{ }^{39}$. In a sheep model of chronic hypertension, left atrial surface area increased by $22 \%{ }^{40}$. However, in these models the role of atrial dilatation itself for the development of a substrate of AF could not be established. For this purpose, several models of chronic atrial dilatation have been developed. Although most of these models display an increased AF vulnerability, they differ considerably in the structural changes associated with atrial dilatation. 

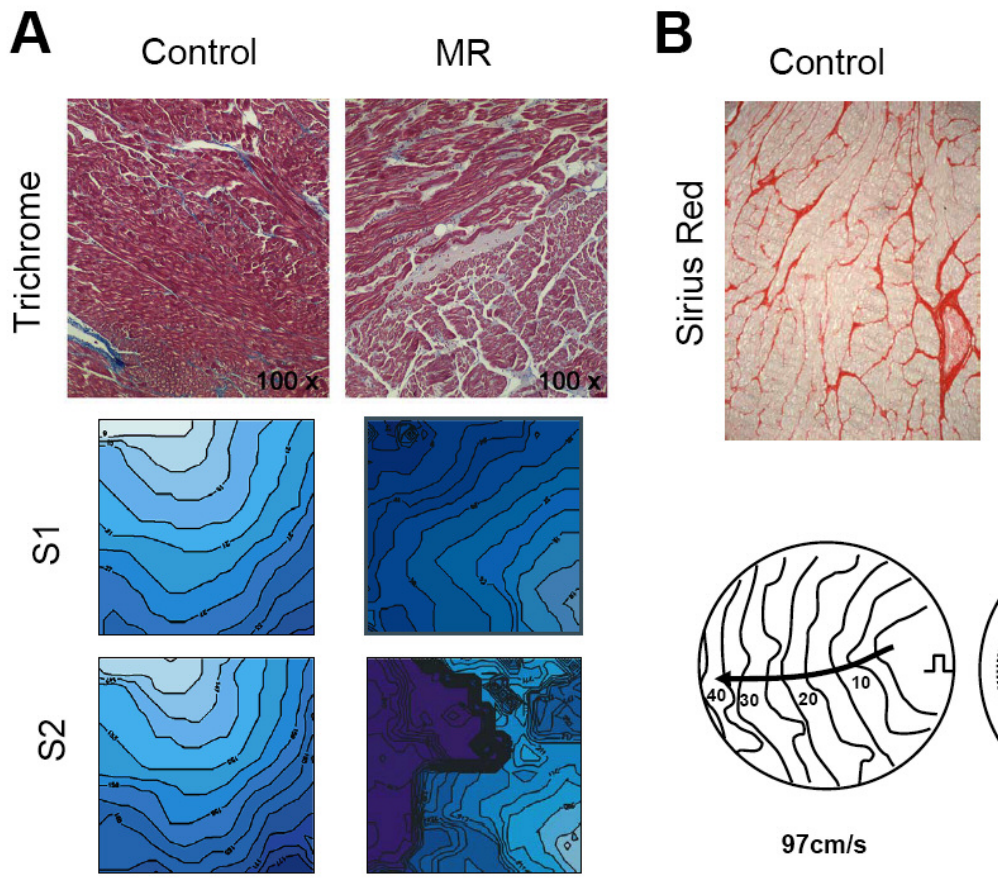

AV block

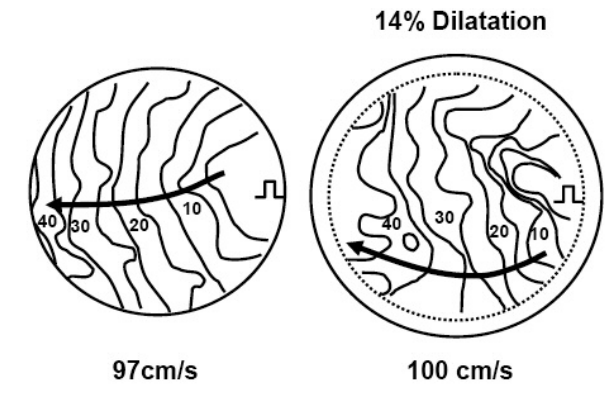

Figure 3: Atrial structure and conduction in animal models of chronic atrial dilatation.

A) Canine model of chronic left atrial dilatation (4-5 weeks) due to mitral regurgitation. Upper panels shows alterations in tissue structure (trichrome staining; myocytes are colored red, fibrosis blue), with areas of fibrosis and inflammatory infiltrates. Lower panels show that in control left atria, both a normal stimulus S1 (interval $350 \mathrm{msec}$ ) and a closely coupled premature stimulus S2 interval (140 msec) conducts homogeneously from the site of stimulation. Although conduction during normal pacing (S1) is still homogeneous, a premature stimulus S2 induced marked slow conduction with a strong wavefront curvature (S1-S1 $=350, S 1-S 2=160 \mathrm{msec}$, from reference ${ }^{41}$ ).

B) Goat model of bi-atrial dilatation due to chronic complete AV block (4 weeks). Upper panels show tissue structure (sirius red staining; myocytes are colored green, 
fibrosis red), illustrating that fibrosis did not increase. During pacing with a cycle length of $200 \mathrm{msec}$, areas of slow heterogeneous conduction were apparent in AV block goats (from reference ${ }^{42}$ ).

The first animal model of chronic atrial dilatation was described by Boyden and Hoffman ${ }^{43}$. Partial occlusion of the pulmonary artery and partial avulsion of the tricuspid valve in dogs resulted in enlarged and structurally remodeled right atria characterized by cellular hypertrophy and increased interstitial fibrosis. These enlarged atria were more vulnerable to AF. However, this was not due to electrical remodeling since action potentials were not significantly different from control.

In a canine model of mitral regurgitation (MR) the left atrium becomes significantly enlarged during the first minutes after MR, thereafter increasing more gradually to $129 \%$ of the baseline width during the first 3 weeks of MR (an area increase of $67 \%$ ) 41. Histological analysis demonstrated areas of inflammatory infiltrates and slightly increased fibrosis in these MR dogs (figure 3A, upper panel), while the distribution of the gap junction proteins connexin (Cx)40 and Cx43 was not markedly different and myocyte size did not increase ${ }^{41,44}$. The increased duration of AF episodes in MR dogs could not be explained by a decrease in wavelength, because the AERP was homogeneously increased without a difference in the overall conduction velocity. High-resolution optical mapping revealed that conduction heterogeneity in LA was significantly increased during pacing at short cycle lengths and premature stimuli (figure $3 \mathrm{~A}$, lower panel) ${ }^{45}$. Together, these results indicate that the increased AF vulnerability in MR dogs was caused by structural changes leading to increased heterogeneity in conduction. 
In another model of chronic atrial dilatation, the time course of atrial dilatation and stability of AF was studied ${ }^{42}$. Chronic complete AV-block in goats resulting in a slow idioventricular rhythm and volume overload of the ventricles caused a progressive atrial enlargement and hypertrophy of the atrial myocytes. In a time period of 4 weeks of AV-block, the mean RA diameter gradually increased by $14 \%$, corresponding to an area increase of $29 \%$. Interestingly, no signs of atrial fibrosis were found in goats with chronic AV-block (figure 3B, upper panel). Atrial dilatation was paralleled by a gradual increase in AF stability while the AERP and the dispersion of AERP remained constant. During sinus rhythm and slow pacing, atrial conduction velocity was slightly increased. However, atrial mapping during pacing at higher rates showed a higher incidence of areas with slow conduction in dilated atria (figure 3B, lower panel). This local conduction slowing was not caused by increased fibrosis and qualitative assessment of the expression of $\mathrm{Cx} 40$ and $\mathrm{C} \times 43$ also did not show marked alterations.

In a rabbit model with an arterio-venous shunt leading to chronic overload with an estimated increase in left atrial surface area of $112 \%$, atrial conduction velocity was significantly decreased by about $30 \%{ }^{46}$. Atrial tachy-arrhythmias could be induced by burst pacing in 7 out of 8 perfused hearts from these 'shunt' rabbits, but in none of the control animals. In the majority of cases, the arrhythmias arose from the posterior left atrium and displayed either a focal pattern of origin or a single reentrant circuit. In this model, the expression levels of both $\mathrm{C} \times 40$ and $\mathrm{C} \times 43$ protein were significantly reduced ${ }^{47}$.

In summary, animal models have demonstrated that chronic atrial dilatation causes an increased stability of AF. This occurs in the absence of AERP shortening and 
involves increased heterogeneity of conduction. Proarrhythmic factors that have been implicated as contributors to the substrate for AF in these models include fibrosis, myocyte hypertrophy, altered connexin protein distribution and the increase in atrial size itself.

The estimated increase in atrial surface area varied between 30 and $110 \%$ in the models mentioned above. In most of these models, neither the AERP nor the conduction velocity was affected. Thus, if the dilated atria formed a homogeneous medium, the number of reentrant waves which could coexist would increase slightly, in proportion with the increased surface area. However, many models of atrial dilatation have an increased heterogeneity of atrial conduction in common. One structural alteration that can produce heterogeneous conduction is fibrosis. Atrial fibrosis has been observed in canine models of dilatation due to valvular insufficiency, ${ }^{41,43}$ but was absent in the goat model of AV block ${ }^{42}$. The difference between models may be related to the time course of atrial dilatation. In the goat AV block model, the slower time course may allow atrial myocytes to adapt and undergo cellular hypertrophy. In the canine models, partial valvular avulsion causes a sudden onset of dilatation, which may lead to acute damage and subsequent replacement fibrosis. Atrial fibrosis may in itself be sufficient to increase AF vulnerability. In mice with selective atrial fibrosis due to overexpression of TGFb1, but not in wildtype littermates, episodes of AF could be induced (figure 4) ${ }^{48}$. Spach et al have demonstrated that in canine and human atrial trabeculae, interstitial fibrosis leads to pronounced delays during transverse propagation, which may allow reentry to occur in very small circuits ${ }^{49-51}$. 
In both the dog model of left atrial dilatation due to mitral regurgitation ${ }^{44}$ and the goat model of bi-atrial dilatation due to $\mathrm{AV}$ block, ${ }^{42}$ no alteration in the distribution pattern of $\mathrm{C} \times 40$ and $\mathrm{C} \times 43$ was observed using immunohistochemistry, although protein levels were not determined in these studies. In a rabbit model of chronic overload with pronounced atrial dilatation, the overall levels of gap junction proteins were reduced ${ }^{47}$. By contrast, Takeuchi et al have reported that the level of Cx40 and Cx43 was not different between SR patients, patients with atrial dilatation and SR and patients with atrial dilatation and $\mathrm{AF}^{52}$. However, confocal microscopy showed a redistribution of $\mathrm{Cx} 43$ within the intercalated discs at the end-to-end connections between atrial myocytes. In the hypertrophied myocytes of patients with atrial dilatation, $\mathrm{Cx} 43$ was shifted to the periphery of the intercalated disc. Although the contribution of altered connexin expression and distribution to AF vulnerability in dilated atria is uncertain at this point, some of the electrophysiological effects of chronic dilatation can be reversed by the gap junction modifier rotigaptide. In a dog model of left atrial dilatation due to mitral regurgitation, rotigaptide increased conduction velocity and reduced AF stability ${ }^{44}$. Similarly, conduction velocity in the rabbit model of chronic volume overload was increased by rotigaptide, although in that model, rotigaptide did not affect the vulnerability to tachy-arrhythmias ${ }^{47}$. 


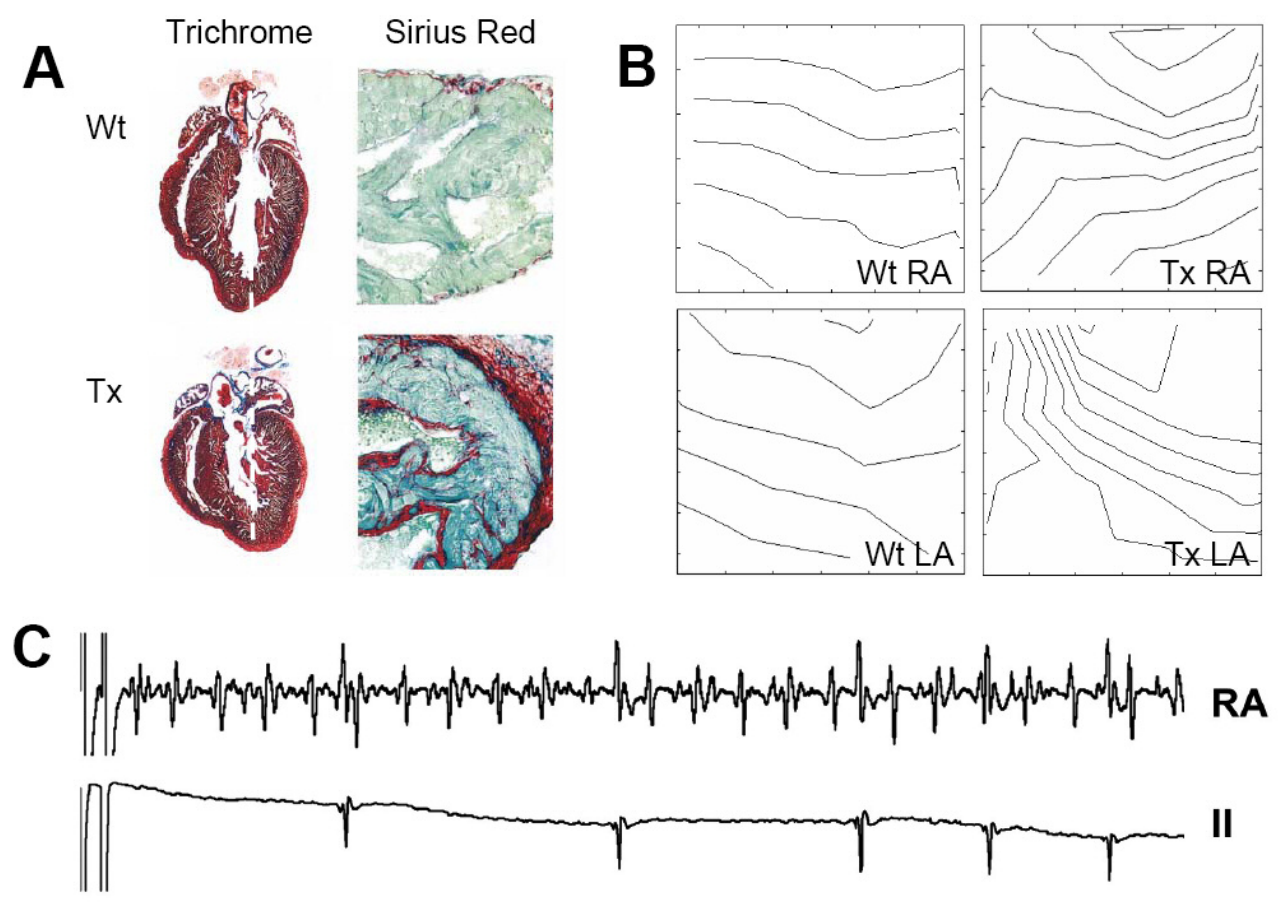

Figure 4: Selective atrial fibrosis in $M H C-C y s^{33}$ ser TGFß1 mice (Tx) leads to heterogeneous conduction and increased AF vulnerability. Images of wild type mice (Wt) are shown for comparison.

A) Over expression of a constitutively active form of TGFß1 does not affect cardiac gross anatomy and does not induce ventricular fibrosis (first column). However, pronounced fibrosis is induced in the atria (sirius red staining; myocytes are colored green, fibrous tissue red).

B) Mapping with a $4 \times 4$ epicardial electrode array revealed heterogeneous atrial conduction in Tx mice. C) In 50\% of Tx and 0\% of Wt mice, episodes of AF could be induced by burst pacing. Upper trace depicts rapid and irregular atrial activity during an induced episode of $A F$ (from reference ${ }^{48}$ ). 
Normal atrial myocytes have a high degree of electrical coupling. The extent to which electrical coupling has to decrease in order to affect wavefront propagating is still a matter of discussion. Some information on the relation between Cx expression and conduction velocity has been obtained in transgenic mouse models. Ventricular myocytes express only Cx43 and in Cx43 heterozygote mice that express $50 \%$ of the normal level of $\mathrm{Cx} 43$, the ventricular macroscopic conduction velocity was not affected ${ }^{53}$. Atrial myocytes express both $\mathrm{C} \times 40$ and $\mathrm{C} \times 43$, and these connexins are likely to form heteromeric (mixed) gap junction channels ${ }^{54}$. In Cx40 heterozygote mice, atrial conduction velocity was not affected, but in Cx40 knock out mice, atrial conduction velocity was reduced by $30 \%{ }^{55}$. The effect of reduced electrical coupling on propagation has been investigated in detail in mathematical models. 'Coupling clamp' experiments, in which two individual atrial myocytes were coupled via a computer, have demonstrated that a coupling conductance of $0.65 \mathrm{nS}$ (nanosiemens) is sufficient for action potential transfer between two cells (corresponding to 3-6 gap junction channels) ${ }^{56}$. In reality, the average coupling conductance between atrial myocytes is $170 \mathrm{nS}^{57}$. In cardiac tissue however, delays between adjacent myocytes will add up, resulting in a decreased conduction velocity. Using a mathematical model of a strand of ventricular myocytes, Shaw and Rudy have shown that a reduction in gap junctional coupling can cause slow propagation with a high safety factor (i.e. unlikely to block) ${ }^{58}$. Spach and Heidlage have presented a model of a 2-dimensional sheet of myocytes that incorporated a detailed topology of longitudinal and transverse gap junctions between myocytes ${ }^{59}$. In this model, activation time delays were observed during transverse propagation even with normal coupling conductances. 
The studies on transgenic mice and mathematical models discussed above have investigated propagation during slow pacing. It is conceivable that reduced $\mathrm{Cx}$ expression has a more pronounced effect during the high and irregular activation rates of AF. In addition, atrial dilatation may cause both an alteration in $\mathrm{Cx}$ distribution and an increase in cell size. Myocyte hypertrophy has been observed in a dog model of right atrial enlargement due to combined pulmonary stenosis and tricuspid insufficiency ${ }^{43}$ and in the goat model of $A V$ block ${ }^{42}$. The effect of cellular hypertrophy on atrial conduction is difficult to predict. From cable equations, it might be expected that an increase in myocyte width would lead to an increase in conduction velocity. Indeed, in a mathematical model of ventricular hypertrophy, an increase in macroscopic conduction velocity was observed ${ }^{60}$. However, using a more detailed model that took the non-uniform distribution of gap junction around myocytes into account, Spach et al calculated that an increase in cell size would lead to more pronounced propagation delays between myocytes during transverse propagation ${ }^{61,62}$. In fact, cell size had a larger effect in this model on the anisotropy of conduction than the distribution pattern of gap junctional plaques around the myocyte. In another modeling study, Wilders and Jongsma showed that the electrostatic interactions between neighboring gap junction channels produces a decrease in the effective junctional conductance in a gap junctional plaque. Thus, the conductance of large gap junctional plaques is lower than would be expected based on the number of gap junction channels present ${ }^{63}$. Hall and Gourdie have also provided arguments that the packing arrangement of gap junction channels within the intercalated disc can affect junctional resistance ${ }^{64}$. Taking these considerations into account, the redistribution of $\mathrm{Cx}$ proteins within intercalated discs 
that has been described for myocytes from dilated human atria may significantly affect action potential propagation ${ }^{52}$.

\section{'Breakthroughs': Focal Activity or Transmural Conduction?}

In a variety of animal models as well as in patients with atrial dilatation, local heterogeneities in conduction have been demonstrated to underlie the high AF susceptibility in structurally remodeled atria $41,42,65,66$. The structural remodeling process results in progressive electrical dissociation between muscle bundles, as demonstrated by Spach et al. in atrial trabeculae of patients with varying age ${ }^{67}$. Local heterogeneities in conduction have been described during rapid pacing or premature stimulation and certainly have the potential to initiate episodes of $\mathrm{AF}^{45}$. However, studies on wavefront propagation during AF are still sparse.

The first mapping study of human AF induced by burst pacing was performed in 1991 by Cox et al. in patients with Wolff-Parkinson-White syndrome undergoing cardiac surgery for interruption of the accessory atrio-ventricular pathway ${ }^{68}$. The study supported reentry as the basic underlying mechanism sustaining the arrhythmia, demonstrating non-uniform conduction around regions of bi-directional block resulting in multiple discrete wavefronts, and suggested that the majority of the atrial reentrant circuits during AF were functional in nature. Konings et al. showed that in patients with acute AF induced by rapid atrial pacing, atrial rate and degree of complexity of activation were quite variable. Both, uniform propagation of single broad wavefronts, as well as highly disorganized activation by multiple wavelets of various sizes were observed ${ }^{69}$. While in this study the majority of the wavefronts were still broad (in the order of centimeters) with relative uniform spread of 
activation, recent data of de Groot et al. suggest a much more complex pattern of activation in patients with chronic AF undergoing mitral valve surgery. Multiple lines of block are present in both right and left atria resulting in very narrow wavefronts (in the order of millimeters) which greatly adds to the complexity of the propagation pattern and the stability of AF. Because of narrowing of the wavefronts, many more fibrillation waves are simultaneously present in the atria. This data supports earlier reports from clinical studies in patients with either paroxysmal or chronic AF ${ }^{70,71} .64$ pole basket catheters were used in both studies to analyze activation patterns in human right atria. Despite the limited spatial resolution of the catheters used, both studies suggest that compared to paroxysmal AF there was a significantly higher number of endocardial breakthrough events and a more pronounced complexity of $A F$ in patients with chronic $A F$.

The structural remodeling process might not only lead to longitudinal electrical dissociation between muscle bundles in the epicardial plane, but may also result in increased endo-epicardial dissociation. Electrical disruption between the thin epicardial layer and the endocardial bundle network will transform the atrial wall into a complex 3-dimensional medium for wavefront propagation. However, most experimental and clinical mapping studies of AF have only determined the spread of activation in either the epicardial or the endocardial plane. Yet, these studies clearly suggest that transmural conduction of fibrillation waves might frequently occur during AF. In high density mapping analysis of acute AF in patients with Wolff-ParkinsonWhite syndrome undergoing surgical disruption of their accessory pathway Konings et al. demonstrated that fibrillation waves occasionally appeared in the middle of the mapping area ${ }^{69}$. These fibrillation waves could not be explained by propagation 
within the epicardial plane and were classified as 'breakthroughs' originating from deeper layers of the atrial wall. During acutely induced AF in normal atria, the incidence of such epicardial breakthroughs was low and only comprised approximately $1.5 \%$ of all fibrillation waves ${ }^{69}$. In patients with persistent AF, Holm et al. observed frequent episodes of focal spread of activation in the right atrium. The number of activations per episode varied considerably and the AF cycle length during focal activation did not differ from the average AF cycle length ${ }^{72}$. The authors concluded that this conduction pattern was most consistent with epicardial activation originating from endocardial fibers activated out of phase. Schuessler et al were the first to show that, while endo-epicardial activation time differences were small during sinus rhythm or slow atrial pacing, there were larger differences in endo-epicardial activation times during premature stimulation or AF sustained by intravenous acetylcholine infusion ${ }^{73}$. The largest endo-epicardial time differences were found in the thicker parts of the atria where pectinate muscles were running under the epicardial layer or in thinner regions with transmural differences in fiber orientation. 
AAF

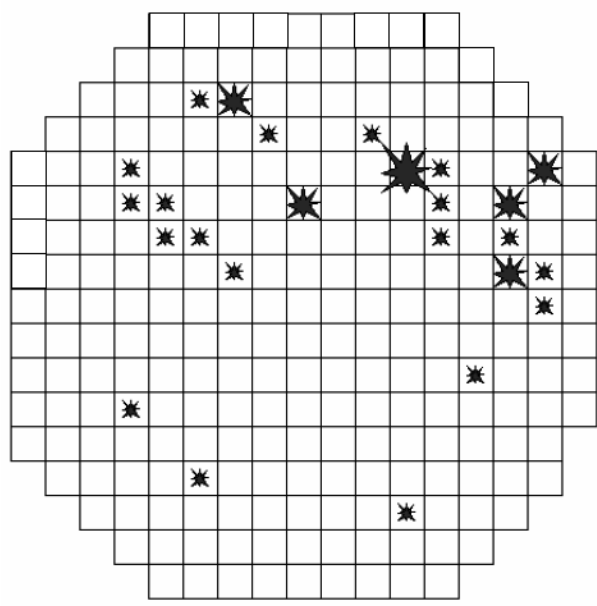

CAF

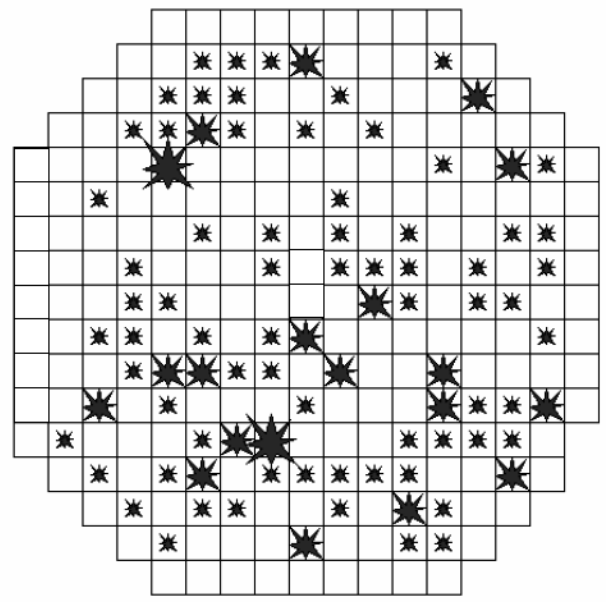

Figure 5: Spatial distribution of epicardial breakthroughs (size of the red stars correlates with total number of breakthroughs) in one patient without structural heart disease and acute $A F(A A F)$ and one patient with valvular heart disease and chronic $A F$ (CAF). Recordings were obtained during 8 seconds of $A F$ at the right atrial free wall (mapping area $4 \times 4 \mathrm{~cm}, 256$ electrodes). The median AFCL was comparable (AAF: $162 \mathrm{~ms}, \mathrm{CAF}: 160 \mathrm{~ms}$ ). In the CAF patient, the incidence of epicardial breakthrough was higher and occurred at more sites within the mapping area.

The incidence of epicardial breakthroughs in patients with acute and chronic AF was recently measured by de Groot et al. ${ }^{10}$. In the right atria of patients with chronic AF suffering from valvular heart disease, epicardial breakthrough was found to be $2-3$ times more frequent than in patients with sinus rhythm and acutely induced AF (figure 5). The variability of the $\mathrm{AFCl}$ was increased suggesting a prolongation of the excitable gap in patients with dilated atria. The occurrence of epicardial 
breakthroughs contributed to the increased $\mathrm{AFCl}$ variability and correlated with the incidence of intra-atrial conduction block ${ }^{10}$. S-wave predominance in epicardial fibrillation electrograms point towards a leading role of the epicardial layer in propagation of the fibrillation waves ${ }^{74}$. The data of de Groot et al. suggest that the duration of $\mathrm{AF}$ and the complexity of the AF substrate in chronically dilated atria correlate with an increased incidence of conduction block within the epicardial layer resulting in a wider excitable gap and a higher degree of electrical endo-epicardial dissociation. The more the subepicardial layer and the endocardial bundle network are activated out of phase and the longer the excitable gap, the higher is the chance that fibrillation waves will 'cross-over' from the endo- to the epicardium or vice versa. Other authors have advocated an ectopic mechanism to be responsible for breakthroughs. In a variety of experimental settings, both automaticity as well as triggered activity have been reported to underlie the generation of new wavefronts. In most of these studies, atrial preparations were exposed to high concentrations of beta-adrenergic agents to increase the SR $\mathrm{Ca}^{2+}$ load.

In rabbit right atrial preparations, conventional mapping analysis revealed that ryanodine can induce a shift of the pacemaker activity from the sinus node to the right PV atrial junction. Under these circumstances rapid pacing can induce selfterminating bursts of spontaneous action potentials which could be inhibited by depletion of the SR or blockade of the sarcolemmal $\mathrm{Na}+/ \mathrm{Ca}^{2+}$-exchanger ${ }^{75}$. Thus, at least under certain circumstances - the PV myocardial sleeves have the potential to generate spontaneous activity. Triggered activity has also been suggested to underlie ectopic activity in atrial tissue. In canine superfused PV sleeves, shortening of the action potential by acetylcholine together with enhancement of the $\mathrm{Ca}^{2+}$ 
transient with norepinephrine or post pause potentiation provoked early afterdepolarizations (EADs) inducing rapid trains of spontaneous action potentials ${ }^{76}$. In the presence of acetylcholine, such 'late phase 3 EADs' were capable to re-induce AF within a couple of seconds after termination of AF or rapid atrial pacing in coronary-perfused canine right atria ${ }^{77}$. This phenomenon might contribute to immediate recurrences of $A F$ in patients and has very recently been suggested to underlie the onset of PV firing in dogs and men ${ }^{78}$.

Many of these experimental studies use focal spread of activation as the central argument for the existence of ectopic activity ${ }^{78-81}$. In dogs with pacing-induced CHF, rapid atrial pacing induced sustained atrial tachycardia with focal spread of activation mostly originating from the crista terminalis or from the PV area ${ }^{80}$. The radial activation pattern was found in endocardial as well as epicardial mapping experiments ${ }^{82}$. Chou and colleagues studied Langendorff-perfused canine left atrial and PV preparations. After ryanodine and isoproterenol infusion rapid atrial pacing induced episodes of focal discharge originating from the PVs. Dual camera optical mapping demonstrated that during some episodes a rise of intracellular $\mathrm{Ca}^{2+}$ was detectable just before the rise of membrane voltage.

This finding supports the concept of atrial tachycardia caused by DADs due to spontaneous $\mathrm{Ca}^{2+}$ release ${ }^{81}$.

Further support for a role of triggered activity in the generation of fibrillation wavefronts comes from studies on alterations of intracellular $\mathrm{Ca}^{2+}$ handling in fibrillating atria. Vest et al. demonstrated an enhanced open probability of the ryanodine receptors in atrial myocardium of dogs undergoing rapid atrial pacing as well as of patients with sustained $\mathrm{AF}^{83}$. The authors conclude that these alterations 
might facilitate spontaneous $\mathrm{Ca}^{2+}$ release events from the sarcoplasmic reticulum which in turn might induce DADs and possibly trigger action potentials. In line with this, Hove-Madson et al. reported an increase of the frequency of $\mathrm{Ca}^{2+}$ sparks (elementary $\mathrm{Ca}^{2+}$ release events) in atrial myocytes isolated from the right atria of patients with sustained $\mathrm{AF}^{84}$. The $\mathrm{Ca}^{2+}$ load of the sarcoplasmic reticulum was unaltered which supports the hypothesis that the increase in spontaneous $\mathrm{Ca}^{2+}$ release was due to a change of the intrinsic properties of the ryanodine channels. This interesting hypothesis, however, raises some questions regarding the relevance of these cellular observations for the pathophysiology in-vivo. For instance, it is unclear why despite the fact that in atrial myocytes isolated from the right atrial appendages of patients with $\mathrm{AF}$ the $\mathrm{Ca}^{2+}$ spark frequency is enhanced, ectopic activity originating from this region is a rare phenomenon. Similarly, dogs undergoing rapid atrial pacing do not develop atrial ectopy although the open probability of the atrial ryanodine channels is increased. Also, increased open probability alone only transiently enhances spontaneous $\mathrm{Ca}^{2+}$ release from the sarcoplasmic reticulum since increased release would reduce the $\mathrm{SR} \mathrm{Ca}^{2+}$ load. Additional stimulation of the $\mathrm{Ca}^{2+}$ re-uptake rate is required to maintain $\mathrm{Ca}^{2+}$ load and produce a sustained enhancement of $\mathrm{Ca}^{2+}$ release events ${ }^{85}$. Of note, phosphorylation levels of phospholamban, an inhibitory protein controlling the reuptake of $\mathrm{Ca}^{2+}$ into the sarcoplasmic reticulum by the sarcoplasmic reticulum $\mathrm{Ca}^{2+}$ ATPase (SERCA) has been shown to be increased in atrial myocardium of patients with $\mathrm{AF}^{86}$. Enhanced phosphorylation of phospholamban dissociates the molecule from SERCA and stimulates reuptake of $\mathrm{Ca}^{2+}$ into the sarcoplasmic reticulum possibly increasing $\mathrm{Ca}^{2+}$ load and spontaneous $\mathrm{Ca}^{2+}$ release from the intracellular $\mathrm{Ca}^{2+}$ stores. 
Whatever the underlying mechanisms of breakthroughs are, a central technique to evaluate their origin is simultaneous endo-epicardial high density mapping. In case the fibrillation waves originate from deeper layers of the atrial wall, breakthroughs should be preceded by electrical activation at or close to the opposing electrode on the contralateral side of the atrial wall. If such a correlation cannot be established triggered activity or automaticity are the more likely mechanisms.

We recently performed simultaneous endo-epicardial high density mapping studies in goats instrumented with a right atrial endocardial screw-in electrode and a fibrillation pacemaker. Acute episodes of AF were induced in control animals by short episodes of endocardial burst pacing with $50 \mathrm{~Hz}$. Persistent AF was achieved after 3 weeks of intermittent atrial burst pacing.

Simultaneous endo-epicardial recordings in a goat with persistent AF revealed areas with significant differences in epicardial versus endocardial AF cycle length. Figure 6 shows the mean AF cycle length in ms of a 10 second recording for each electrode of the mapping array and 3 electrogram tracings. It can be seen that while in the epicardial plane (A) and the left upper part of the endocardium (B) AF cycle length was between 80 and $110 \mathrm{~ms}$, it was clearly longer in the right lower endocardial area (C). This phenomenon clearly suggests that the epicardial layer and the endocardial bundle network can become activated out of phase during AF. 
Epicardial AFCL

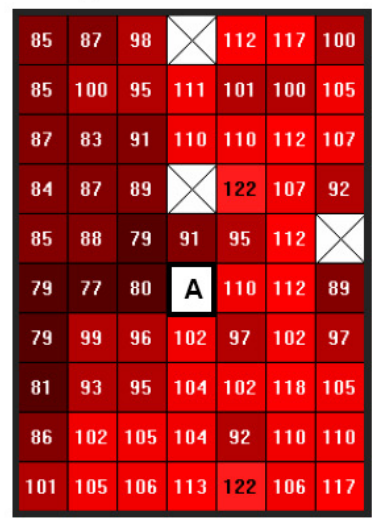

Endocardial AFCL

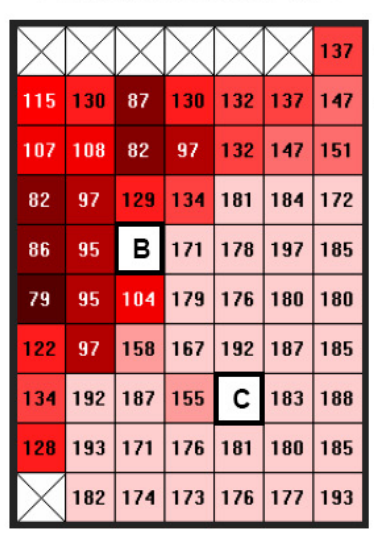

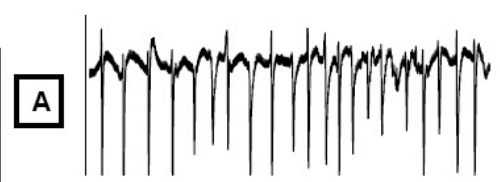
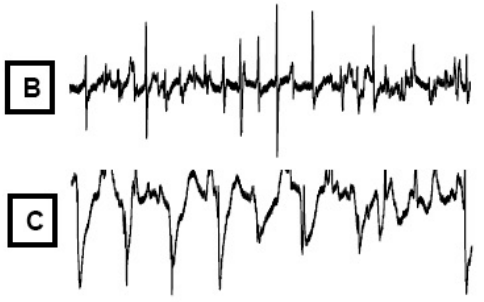

200 ms

Figure 6: Matrix, representing the epicardial (left) and endocardial (right) surface of the mapping tool and 3 representative unipolar electrogram tracings $(A, B$ and $C)$. Numbers in the matrix give the average $A F$ cycle length $(\mathrm{ms})$ per electrode for 50 consecutive beats; Visualizing an endocardial area (C) with significantly longer AF cycle length than the surrounding endocardial (B) and opposing epicardial area (A).

Using the above mentioned mapping technique we could as well demonstrate epicardial focal spread of activation with preceding electrical activity in the opposing endocardial side or vice versa. 


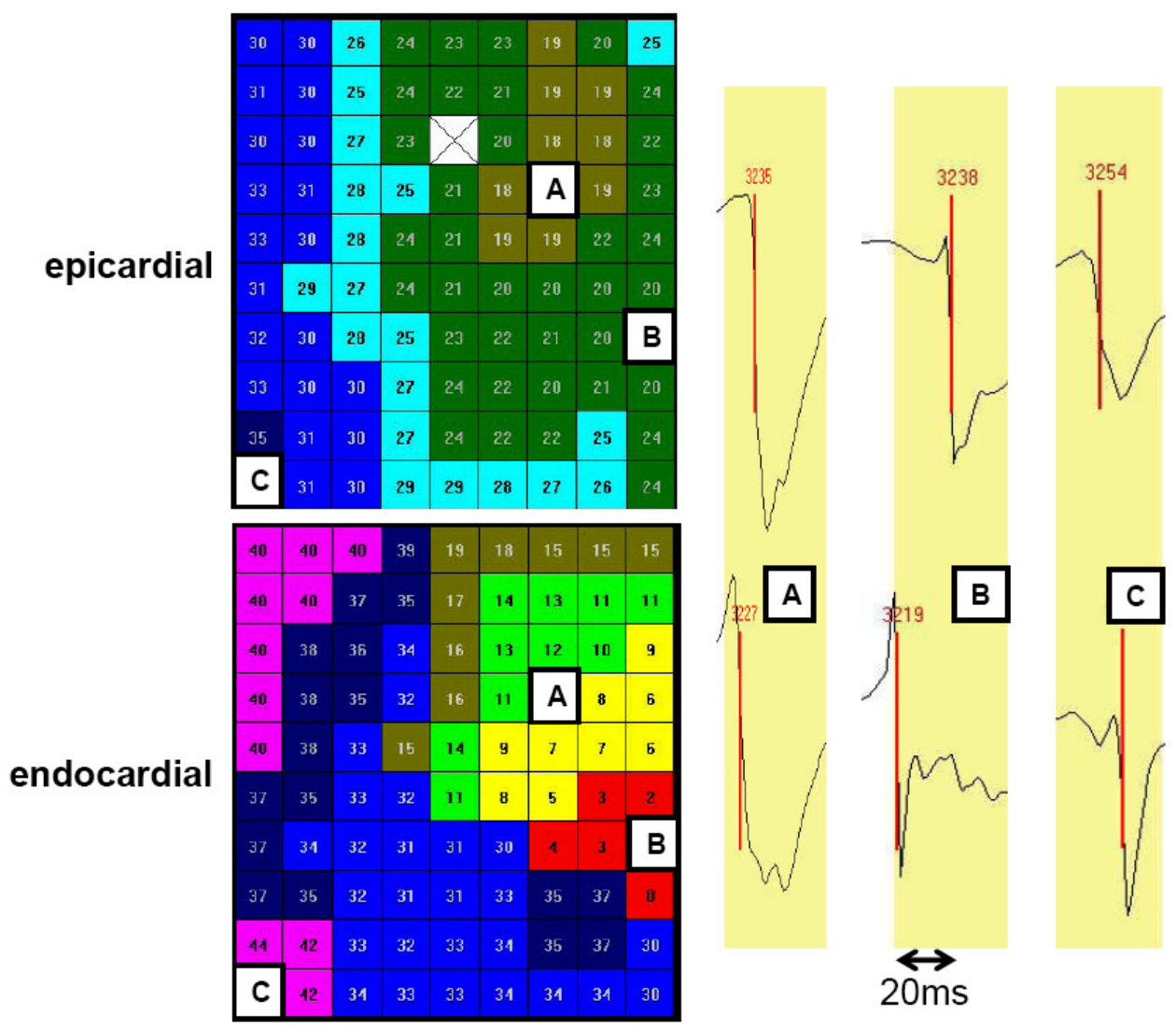

Figure 7: Matrix, representing the epicardial (upper) and endocardial (lower) surface of the mapping tool and 3 representative unipolar electrogram tracing pairs (endocardial/epicardial, $A, B$ and C). Numbers give the activation time (ms) map of one AF-beat visualizing the path taken by the depolarizing wave. $A \Delta t=8 \mathrm{~ms}$ (site of breakthrough), $B \Delta t=19 \mathrm{~ms}$ (earliest activation, endocardium earlier), $C \Delta t=8 \mathrm{~ms}$ (epicardium earlier). 
Figure 7 shows an activation time map with corresponding epicardial / endocardial activation times and examples of electrogram tracings at 3 different corresponding electrode pairs. It demonstrates an example of "focal activity" in the epicardial plane at time point $17 \mathrm{~ms}$ (A). All surrounding epicardial electrodes are activated later demonstrating a radial spread of activation. The corresponding endocardial site is activated $8 \mathrm{~ms}$ earlier (A) and is part of an early endocardial wave entering the mapping area from the lower right side of the mapping electrode. At the entry site of this earlier wave the endo- epicardial activation time difference is $19 \mathrm{~ms}(\mathrm{~B})$. This pronounced dissynchronicity suggests that the preceding endocardial wave activates the epicardial layer via transmural conduction causing a breakthrough pattern at site A. This breakthrough initiates a wave with homogeneous spread of activation within the epicardial mapping area. When this epicardial wave propagates over the lower left endocardial part, which has not been activated by the initial wave, it finally activates the endocardial layer via transmural conduction. This explains why these parts of the endocardium are activated up to $8 \mathrm{~ms}$ later than their corresponding epicardial sites (C). As mentioned above, such a pattern of "breakthrough" can only occur in the setting of significant dissociation of endo-epicardial electric activity. In the experiment mentioned above the mean absolute endo-epicardial activation time difference was $18.5 \mathrm{~ms} \pm 33.1 \mathrm{~ms}$ compared to $7.7 \mathrm{~ms} \pm 13.1 \mathrm{~ms}$ in control goats with acute AF. These data support the hypotheses of de Groot and Houben that during the development of a substrate of AF endo-epicardial dissociation increases due to conduction in the epicardial layer which might facilitate transmural conduction of fibrillation wavefronts. 


\section{Conclusion}

Understanding the mechanisms leading to initiation and perpetuation of $\mathrm{AF}$ is of utmost importance for the development of future therapeutic strategies. In chronically dilated atria, the substrate of $\mathrm{AF}$ is characterized by local conduction disturbances due to electrical dissociation between muscle bundles. Such electrical dissociation might occur within the subepicardial layer and the endocardial bundle network but also between different layers of the atrial wall. In patients with chronically dilated atria due to mitral valve disease, the incidence of focal spread of activation which could not be explained by conduction in the epicardial plane was increased. In theory, these wavefronts might be due to ectopic activity or they might represent transmural conduction of fibrillation waves from deeper layers of the atrial wall. Numerous studies address the mechanism of new wavefront generation using 2 dimensional mapping analysis. Although these studies add valuable information to the existing theories, information concerning conduction of fibrillation waves in the third dimension of the atrial wall is still very limited. In the goat model of AF, breakthroughs were investigated by simultaneous endo-epicardial high density mapping of atrial free walls. With this technique we provide preliminary evidence that endo-epicardial electrical dissociation is present during AF and fibrillation waves, with a focal spread of activation that might have been interpreted as "focal activity", in fact represent transmural conduction originating from deeper layers of the atrial wall. Both, endo-epicardial electrical dissociation and prolongation of the excitable gap appear to promote the 3 dimensional conduction patterns in advanced stages of atrial remodeling. 


\section{References:}

1. Mines GR. On dynamic equilibrium in the heart. J Physiol. 1913;46(4-5):349383.

2. Mayer AG. Rhythmical pulsation in Scyphomedusae I. Carnegie Inst. Wash. Paper. 1906;47.

3. Moe GK, Abildskov JA. Atrial fibrillation as a self-sustaining arrhythmia independent of focal discharge. Am Heart J. 1959;58(1):59-70.

4. Allessie MA, Lammers W, Bonke F, Hollen J. Experimental evaluation of Moe's multiple wavelet hypothesis of atrial fibrillation New York: Grune \& Stratton; 1985.

5. Kumagai K, Khrestian C, Waldo AL. Simultaneous multisite mapping studies during induced atrial fibrillation in the sterile pericarditis model. Insights into the mechanism of its maintenance. Circulation. 1997;95(2):511-521.

6. Jalife J, Berenfeld $\mathrm{O}$, Mansour M. Mother rotors and fibrillatory conduction: a mechanism of atrial fibrillation. Cardiovasc Res. 2002;54(2):204-216.

7. Mandapati R, Skanes A, Chen J, Berenfeld O, Jalife J. Stable microreentrant sources as a mechanism of atrial fibrillation in the isolated sheep heart. Circulation. 2000;101(2):194-199.

8. Mansour M, Mandapati R, Berenfeld O, Chen J, Samie FH, Jalife J. Left-toright gradient of atrial frequencies during acute atrial fibrillation in the isolated sheep heart. Circulation. 2001;103(21):2631-2636.

9. Lazar S, Dixit S, Marchlinski FE, Callans DJ, Gerstenfeld EP. Presence of leftto-right atrial frequency gradient in paroxysmal but not persistent atrial fibrillation in humans. Circulation. 2004;110(20):3181-3186.

10. de Groot NMS. Comparison of epicardial breakthrough of fibrillation waves between patients with acute and chronic atrial fibrillation. In: Mapping and Ablation of Atrial TachyArrhythmias. Maastricht: Fysiologie, Maastricht University; 2006.

11. Sanders $P$, Berenfeld $O$, Hocini $M$, Jais $P$, Vaidyanathan $R$, Hsu LF, Garrigue S, Takahashi Y, Rotter M, Sacher F, Scavee C, Ploutz-Snyder R, Jalife J, Haissaguerre $M$. Spectral analysis identifies sites of high-frequency activity maintaining atrial fibrillation in humans. Circulation. 2005;112(6):789-797.

12. Scherf $D$, Terranova R. Mechanism of auricular flutter and fibrillation. $A m ~ J$ Physiol. 1949;159(1):137-142. 
13. Haissaguerre $M$, Jais $P$, Shah DC, Takahashi A, Hocini M, Quiniou G, Garrigue S, Le Mouroux A, Le Metayer P, Clementy J. Spontaneous initiation of atrial fibrillation by ectopic beats originating in the pulmonary veins. $N \mathrm{Engl}$ J Med. 1998;339(10):659-666.

14. Atienza $F$, Almendral $\mathrm{J}$, Moreno $\mathrm{J}$, Vaidyanathan $\mathrm{R}$, Talkachou $\mathrm{A}$, Kalifa J, Arenal A, Villacastin JP, Torrecilla EG, Sanchez A, Ploutz-Snyder R, Jalife J, Berenfeld $O$. Activation of inward rectifier potassium channels accelerates atrial fibrillation in humans: evidence for a reentrant mechanism. Circulation. 2006;114(23):2434-2442.

15. Patterson E, Jackman WM, Beckman KJ, Lazzara R, Lockwood D, Scherlag BJ, Wu R, Po S. Spontaneous pulmonary vein firing in man: relationship to tachycardia-pause early afterdepolarizations and triggered arrhythmia in canine pulmonary veins in vitro. $J$ Cardiovasc Electrophysiol. 2007;18(10):1067-1075.

16. Wijfels MC, Kirchhof CJ, Dorland R, Allessie MA. Atrial fibrillation begets atrial fibrillation. A study in awake chronically instrumented goats. Circulation. 1995;92(7):1954-1968.

17. Allessie M, Ausma J, Schotten U. Electrical, contractile and structural remodeling during atrial fibrillation. Cardiovasc Res. 2002;54(2):230-246.

18. Schotten $U$, Neuberger $H R$, Allessie MA. The role of atrial dilatation in the domestication of atrial fibrillation. Prog Biophys Mol Biol. 2003;82(1-3):151162.

19. Ausma J, Wijffels M, Thone F, Wouters L, Allessie M, Borgers M. Structural changes of atrial myocardium due to sustained atrial fibrillation in the goat. Circulation. 1997;96(9):3157-3163.

20. Schotten $U$, Allessie $M$. The substrate of atrial fibrillation in chronically dilated atria. In: Kohl FS, ed. Cardiac Mechano-electric feedback and arrhythmias: from pipette to patient; 2005:154-163.

21. Fraser HR, Turner R. Electrocardiography in mitral valvular disease. $\mathrm{Br}$ Heart J. 1955;17(4):459-483.

22. Henry WL, Morganroth J, Pearlman AS, Clark CE, Redwood DR, Itscoitz SB, Epstein SE. Relation between echocardiographically determined left atrial size and atrial fibrillation. Circulation. 1976;53(2):273-279.

23. Benjamin EJ, Levy D, Vaziri SM, D'Agostino RB, Belanger AJ, Wolf PA. Independent risk factors for atrial fibrillation in a population-based cohort. The Framingham Heart Study. Jama. 1994;271(11):840-844. 
24. Vaziri SM, Larson MG, Benjamin EJ, Levy D. Echocardiographic predictors of nonrheumatic atrial fibrillation. The Framingham Heart Study. Circulation. 1994;89(2):724-730.

25. Keren G, Etzion T, Sherez J, Zelcer AA, Megidish R, Miller HI, Laniado S. Atrial fibrillation and atrial enlargement in patients with mitral stenosis. $\mathrm{Am}$ Heart J. 1987;114(5):1146-1155.

26. Sanfilippo AJ, Abascal VM, Sheehan M, Oertel LB, Harrigan P, Hughes RA, Weyman AE. Atrial enlargement as a consequence of atrial fibrillation. A prospective echocardiographic study. Circulation. 1990;82(3):792-797.

27. Dittrich HC, Pearce LA, Asinger RW, McBride R, Webel R, Zabalgoitia M, Pennock GD, Safford RE, Rothbart RM, Halperin JL, Hart RG. Left atrial diameter in nonvalvular atrial fibrillation: An echocardiographic study. Stroke Prevention in Atrial Fibrillation Investigators. Am Heart J. 1999;137(3):494499.

28. Welikovitch L, Lafreniere G, Burggraf GW, Sanfilippo AJ. Change in atrial volume following restoration of sinus rhythm in patients with atrial fibrillation: a prospective echocardiographic study. Can J Cardiol. 1994;10(10):993-996.

29. Hagens VE, Van Veldhuisen DJ, Kamp O, Rienstra M, Bosker HA, Veeger NJ, Tijssen JG, Crijns HJ, Van Gelder IC. Effect of rate and rhythm control on left ventricular function and cardiac dimensions in patients with persistent atrial fibrillation: results from the RAte Control versus Electrical Cardioversion for Persistent Atrial Fibrillation (RACE) study. Heart Rhythm. 2005;2(1):19-24.

30. Therkelsen SK, Groenning BA, Svendsen JH, Jensen GB. Atrial and ventricular volume and function evaluated by magnetic resonance imaging in patients with persistent atrial fibrillation before and after cardioversion. Am J Cardiol. 2006;97(8):1213-1219.

31. Ravelli $F$, Allessie M. Effects of atrial dilatation on refractory period and vulnerability to atrial fibrillation in the isolated Langendorff-perfused rabbit heart. Circulation. 1997;96(5):1686-1695.

32. Bode F, Katchman A, Woosley RL, Franz MR. Gadolinium decreases stretchinduced vulnerability to atrial fibrillation. Circulation. 2000;101(18):2200-2205.

33. Solti $F$, Vecsey $T$, Kekesi $V$, Juhasz-Nagy $A$. The effect of atrial dilatation on the genesis of atrial arrhythmias. Cardiovasc Res. 1989;23(10):882-886.

34. Satoh T, Zipes DP. Unequal atrial stretch in dogs increases dispersion of refractoriness conducive to developing atrial fibrillation. $J$ Cardiovasc Electrophysiol. 1996;7(9):833-842. 
35. Wijffels MC, Kirchhof CJ, Dorland R, Power J, Allessie MA. Electrical remodeling due to atrial fibrillation in chronically instrumented conscious goats: roles of neurohumoral changes, ischemia, atrial stretch, and high rate of electrical activation. Circulation. 1997;96(10):3710-3720.

36. Eijsbouts SC, Majidi M, van Zandvoort M, Allessie MA. Effects of acute atrial dilation on heterogeneity in conduction in the isolated rabbit heart. $J$ Cardiovasc Electrophysiol. 2003;14(3):269-278.

37. Eijsbouts SC, Houben RP, Blaauw Y, Schotten U, Allessie MA. Synergistic action of atrial dilation and sodium channel blockade on conduction in rabbit atria. J Cardiovasc Electrophysiol. 2004;15(12):1453-1461.

38. Kalifa J, Jalife J, Zaitsev AV, Bagwe S, Warren M, Moreno J, Berenfeld O, Nattel S. Intra-atrial pressure increases rate and organization of waves emanating from the superior pulmonary veins during atrial fibrillation. Circulation. 2003;108(6):668-671.

39. Shi Y, Ducharme A, Li D, Gaspo R, Nattel S, Tardif JC. Remodeling of atrial dimensions and emptying function in canine models of atrial fibrillation. Cardiovasc Res. 2001;52(2):217-225.

40. Kistler PM, Sanders P, Dodic M, Spence SJ, Samuel CS, Zhao C, Charles JA, Edwards GA, Kalman JM. Atrial electrical and structural abnormalities in an ovine model of chronic blood pressure elevation after prenatal corticosteroid exposure: implications for development of atrial fibrillation. Eur Heart J. 2006;27(24):3045-3056.

41. Verheule S, Wilson E, Everett Tt, Shanbhag S, Golden C, Olgin J. Alterations in atrial electrophysiology and tissue structure in a canine model of chronic atrial dilatation due to mitral regurgitation. Circulation. 2003;107(20):26152622.

42. Neuberger HR, Schotten $U$, Verheule $S$, Eijsbouts $S$, Blaauw $Y$, van Hunnik A, Allessie M. Development of a substrate of atrial fibrillation during chronic atrioventricular block in the goat. Circulation. 2005;111(1):30-37.

43. Boyden PA, Hoffman BF. The effects on atrial electrophysiology and structure of surgically induced right atrial enlargement in dogs. Circ Res. 1981;49(6):1319-1331.

44. Guerra JM, Everett THt, Lee KW, Wilson E, Olgin JE. Effects of the gap junction modifier rotigaptide (ZP123) on atrial conduction and vulnerability to atrial fibrillation. Circulation. 2006;114(2):110-118.

45. Verheule S, Wilson E, Banthia S, Everett THt, Shanbhag S, Sih HJ, Olgin J. Direction-dependent conduction abnormalities in a canine model of atrial fibrillation due to chronic atrial dilatation. Am J Physiol Heart Circ Physiol. 2004;287(2):H634-644. 
46. Hirose M, Takeishi Y, Miyamoto T, Kubota I, Laurita KR, Chiba S. Mechanism for atrial tachyarrhythmia in chronic volume overload-induced dilated atria. $J$ Cardiovasc Electrophysiol. 2005;16(7):760-769.

47. Haugan K, Miyamoto T, Takeishi Y, Kubota I, Nakayama J, Shimojo H, Hirose M. Rotigaptide (ZP123) improves atrial conduction slowing in chronic volume overload-induced dilated atria. Basic Clin Pharmacol Toxicol. 2006;99(1):7179.

48. Verheule S, Sato T, Everett Tt, Engle SK, Otten D, Rubart-von der Lohe M, Nakajima HO, Nakajima H, Field LJ, Olgin JE. Increased vulnerability to atrial fibrillation in transgenic mice with selective atrial fibrosis caused by overexpression of TGF-beta1. Circ Res. 2004;94(11):1458-1465.

49. Spach MS, Josephson ME. Initiating reentry: the role of nonuniform anisotropy in small circuits. J Cardiovasc Electrophysiol. 1994;5(2):182-209.

50. Spach MS, Boineau JP. Microfibrosis produces electrical load variations due to loss of side-to-side cell connections: a major mechanism of structural heart disease arrhythmias. Pacing Clin Electrophysiol. 1997;20(2 Pt 2):397-413.

51. Spach MS, Heidlage JF, Dolber PC, Barr RC. Mechanism of origin of conduction disturbances in aging human atrial bundles: experimental and model study. Heart Rhythm. 2007;4(2):175-185.

52. Takeuchi S, Akita T, Takagishi Y, Watanabe E, Sasano C, Honjo H, Kodama I. Disorganization of gap junction distribution in dilated atria of patients with chronic atrial fibrillation. Circ J. 2006;70(5):575-582.

53. Morley GE, Vaidya D, Samie FH, Lo C, Delmar M, Jalife J. Characterization of conduction in the ventricles of normal and heterozygous $\mathrm{Cx} 43$ knockout mice using optical mapping. J Cardiovasc Electrophysiol. 1999;10(10):1361-1375.

54. Elenes S, Rubart M, Moreno AP. Junctional communication between isolated pairs of canine atrial cells is mediated by homogeneous and heterogeneous gap junction channels. J Cardiovasc Electrophysiol. 1999;10(7):990-1004.

55. Verheule S, van Batenburg CA, Coenjaerts FE, Kirchhoff S, Willecke K, Jongsma HJ. Cardiac conduction abnormalities in mice lacking the gap junction protein connexin40. J Cardiovasc Electrophysiol. 1999;10(10):13801389.

56. Wang YG, Wagner MB, Kumar R, Goolsby WN, Joyner RW. Fast pacing facilitates discontinuous action potential propagation between rabbit atrial cells. Am J Physiol Heart Circ Physiol. 2000;279(5):H2095-2103.

57. Verheule $\mathrm{S}$, van Kempen $\mathrm{MJ}$, te Welscher $\mathrm{PH}$, Kwak BR, Jongsma HJ. Characterization of gap junction channels in adult rabbit atrial and ventricular myocardium. Circ Res. 1997;80(5):673-681. 
58. Shaw RM, Rudy Y. Ionic mechanisms of propagation in cardiac tissue. Roles of the sodium and L-type calcium currents during reduced excitability and decreased gap junction coupling. Circ Res. 1997;81(5):727-741.

59. Spach MS, Heidlage JF. The stochastic nature of cardiac propagation at a microscopic level. Electrical description of myocardial architecture and its application to conduction. Circ Res. 1995;76(3):366-380.

60. Wiegerinck RF, Verkerk AO, Belterman $\mathrm{CN}$, van Veen TA, Baartscheer A, Opthof T, Wilders R, de Bakker JM, Coronel R. Larger cell size in rabbits with heart failure increases myocardial conduction velocity and QRS duration. Circulation. 2006;113(6):806-813.

61. Spach MS, Heidlage JF, Dolber PC, Barr RC. Electrophysiological effects of remodeling cardiac gap junctions and cell size: experimental and model studies of normal cardiac growth. Circ Res. 2000;86(3):302-311.

62. Spach MS, Heidlage JF, Barr RC, Dolber PC. Cell size and communication: role in structural and electrical development and remodeling of the heart. Heart Rhythm. 2004;1(4):500-515.

63. Wilders R, Jongsma HJ. Limitations of the dual voltage clamp method in assaying conductance and kinetics of gap junction channels. Biophys $J$. 1992;63(4):942-953.

64. Hall JE, Gourdie RG. Spatial organization of cardiac gap junctions can affect access resistance. Microsc Res Tech. 1995;31(5):446-451.

65. Sanders P, Morton JB, Davidson NC, Spence SJ, Vohra JK, Sparks PB, Kalman JM. Electrical remodeling of the atria in congestive heart failure: electrophysiological and electroanatomic mapping in humans. Circulation. 2003;108(12):1461-1468.

66. Li D, Fareh S, Leung TK, Nattel S. Promotion of atrial fibrillation by heart failure in dogs: atrial remodeling of a different sort. Circulation. 1999;100(1):87-95.

67. Spach MS, Dolber PC. Relating extracellular potentials and their derivatives to anisotropic propagation at a microscopic level in human cardiac muscle. Evidence for electrical uncoupling of side-to-side fiber connections with increasing age. Circ Res. 1986;58(3):356-371.

68. Cox JL, Canavan TE, Schuessler RB, Cain ME, Lindsay BD, Stone C, Smith PK, Corr PB, Boineau JP. The surgical treatment of atrial fibrillation. II. Intraoperative electrophysiologic mapping and description of the electrophysiologic basis of atrial flutter and atrial fibrillation. $J$ Thorac Cardiovasc Surg. 1991;101(3):406-426. 
69. Konings KT, Kirchhof CJ, Smeets JR, Wellens HJ, Penn OC, Allessie MA. High-density mapping of electrically induced atrial fibrillation in humans. Circulation. 1994;89(4):1665-1680.

70. Zrenner B, Ndrepepa G, Karch MR, Schneider MA, Schreieck J, Schomig A, Schmitt C. Electrophysiologic characteristics of paroxysmal and chronic atrial fibrillation in human right atrium. J Am Coll Cardiol. 2001;38(4):1143-1149.

71. Ravelli F, Faes L, Sandrini L, Gaita F, Antolini R, Scaglione M, Nollo G. Wave similarity mapping shows the spatiotemporal distribution of fibrillatory wave complexity in the human right atrium during paroxysmal and chronic atrial fibrillation. J Cardiovasc Electrophysiol. 2005;16(10):1071-1076.

72. Holm M, Johansson R, Brandt J, Luhrs C, Olsson SB. Epicardial right atrial free wall mapping in chronic atrial fibrillation. Documentation of repetitive activation with a focal spread--a hitherto unrecognised phenomenon in man. Eur Heart J. 1997;18(2):290-310.

73. Schuessler RB, Kawamoto T, Hand DE, Mitsuno M, Bromberg BI, Cox JL, Boineau JP. Simultaneous epicardial and endocardial activation sequence mapping in the isolated canine right atrium. Circulation. 1993;88(1):250-263.

74. Houben RP, de Groot NM, Smeets JL, Becker AE, Lindemans FW, Allessie MA. S-wave predominance of epicardial electrograms during atrial fibrillation in humans: indirect evidence for a role of the thin subepicardial layer. Heart Rhythm. 2004;1(6):639-647.

75. Honjo H, Boyett MR, Niwa R, Inada S, Yamamoto M, Mitsui K, Horiuchi T, Shibata N, Kamiya K, Kodama I. Pacing-induced spontaneous activity in myocardial sleeves of pulmonary veins after treatment with ryanodine. Circulation. 2003;107(14):1937-1943.

76. Patterson E, Lazzara R, Szabo B, Liu H, Tang D, Li YH, Scherlag BJ, Po SS. Sodium-calcium exchange initiated by the $\mathrm{Ca} 2+$ transient: an arrhythmia trigger within pulmonary veins. J Am Coll Cardiol. 2006;47(6):1196-1206.

77. Burashnikov A, Antzelevitch C. Late-phase 3 EAD. A unique mechanism contributing to initiation of atrial fibrillation. Pacing Clin Electrophysiol. 2006;29(3):290-295.

78. Patterson E, Jackman WM, Beckman KJ, Lazzara R, Lockwood D, Scherlag BJ, Wu R, Po S. Spontaneous Pulmonary Vein Firing in Man: Relationship to Tachycardia-Pause Early Afterdepolarizations and Triggered Arrhythmia in Canine Pulmonary Veins In Vitro. J Cardiovasc Electrophysiol. 2007.

79. Hirose M, Laurita KR. Calcium-mediated triggered activity is an underlying cellular mechanism of ectopy originating from the pulmonary vein in dogs. Am J Physiol Heart Circ Physiol. 2007;292(4):H1861-1867. 
80. Stambler BS, Fenelon G, Shepard RK, Clemo HF, Guiraudon CM. Characterization of sustained atrial tachycardia in dogs with rapid ventricular pacing-induced heart failure. J Cardiovasc Electrophysiol. 2003;14(5):499507.

81. Chou CC, Nihei M, Zhou S, Tan A, Kawase A, Macias ES, Fishbein MC, Lin SF, Chen PS. Intracellular calcium dynamics and anisotropic reentry in isolated canine pulmonary veins and left atrium. Circulation. 2005;111(22):2889-2897.

82. Stambler BS. Atrial tachycardia and congestive heart failure: new insights from an old experimental model. J Cardiovasc Electrophysiol. 2004;15(8):933935.

83. Vest JA, Wehrens XH, Reiken SR, Lehnart SE, Dobrev D, Chandra P, Danilo $P$, Ravens U, Rosen MR, Marks AR. Defective cardiac ryanodine receptor regulation during atrial fibrillation. Circulation. 2005;111(16):2025-2032.

84. Hove-Madsen L, Llach A, Bayes-Genis A, Roura S, Rodriguez Font E, Aris A, Cinca J. Atrial fibrillation is associated with increased spontaneous calcium release from the sarcoplasmic reticulum in human atrial myocytes. Circulation. 2004;110(11):1358-1363.

85. Venetucci LA, Trafford AW, Eisner DA. Increasing ryanodine receptor open probability alone does not produce arrhythmogenic calcium waves: threshold sarcoplasmic reticulum calcium content is required. Circ Res. 2007;100(1):105-111.

86. El-Armouche A, Boknik P, Eschenhagen T, Carrier L, Knaut M, Ravens U, Dobrev D. Molecular determinants of altered $\mathrm{Ca} 2+$ handling in human chronic atrial fibrillation. Circulation. 2006;114(7):670-680. 



\title{
Chapter 3
}

\section{Time Course and Mechanisms of}

\section{Endo-epicardial Electrical Dissociation}

\section{during Atrial Fibrillation in the Goat}

\author{
Jens Eckstein ${ }^{1,2}$, MD; Bart Maesen ${ }^{1}, \mathrm{MD}$; Dominik Linz ${ }^{1}$, MD; Stef Zeemering ${ }^{1}$, \\ MSc; Arne van Hunnik ${ }^{1}$, BSc; Sander Verheule ${ }^{1}$, PhD; Maurits Allessie ${ }^{1}, \mathrm{MD}, \mathrm{PhD}$; \\ Ulrich Schotten ${ }^{1}, \mathrm{MD}, \mathrm{PhD}$
}

1: Cardiovascular Research Institute, Deptartment of Physiology, Maastricht University, Maastricht, The Netherlands 2: Dept. of Medicine, Universityhospital Basel, Basel, Switzerland

Published in: Cardiovasc Res. 2011;89(4):816-824. 


\section{Abstract}

Aims: To determine the degree and mechanisms of endo-epicardial dissociation of electrical activity during atrial fibrillation (AF) and endo-epicardial differences in atrial electrophysiology at different stages of atrial remodeling.

Methods and Results: Simultaneous high-density endo-epicardial mapping of AF was performed on left atrial free walls of goats with acute AF, after 3 weeks and 6 months of AF (all $n=7$ ). Endo-epicardial activation time differences and differences in direction of conduction vectors were calculated, endocardial and epicardial effective refractory periods (ERP) were determined, and fractionation of electrograms was quantified.

Histograms of endo-epicardial activation time differences and differences in direction of conduction vectors revealed two distinct populations, i.e. dissociated and nondissociated activity. Dyssynchronous activity (dissociated in time) increased from $17 \pm 7 \%$ during acute $\mathrm{AF}$ to $39 \pm 17 \%$ after 3 weeks, and $68 \pm 13 \%$ after 6 months of $\mathrm{AF}$. Dissociation was more pronounced in thicker parts of the atrial wall (thick: $49.3 \pm 21.4 \%$, thin: $42.2 \pm 19.0 \%, p<0.05)$. At baseline, endocardial ERPs were longer compared to epicardial ERPs ( $\Delta \mathrm{ERP} 21.8 \pm 18 \mathrm{~ms}, p<0.001)$. This difference was absent after 6 months of AF. The percentage of fractionated electrograms during rapid pacing increased from $9.4 \pm 1.9 \%$ (baseline) to $18.6 \pm 0.6 \%$ (6 months). 
Conclusion: During AF, pronounced dissociation of electrical activity occurs between the epicardial layer and the endocardial bundle network. The increase in dissociation is due to progressive uncoupling between the epicardial layer and the endocardial bundles and correlates with increasing stability and complexity of the AF substrate. 


\section{Introduction}

Atrial fibrillation (AF) is a progressive arrhythmia ${ }^{1}$. Mechanisms underlying the increasing duration of AF episodes and the loss of efficacy of current therapeutic interventions over time have been subject of extensive research ${ }^{2-4}$. Shortening of refractoriness and wavelength certainly contribute to, but cannot fully explain increased AF stability ${ }^{4,5}$. Disruption of electrical side-to-side connections between muscle bundles resulting in longitudinal dissociation of electrical activity has been identified as a key element of the development of a substrate of $A F^{6-8}$. Whether such dissociation of electrical activity also occurs between the epicardial layer and the endocardial bundle network and how this endo-epicardial electrical dissociation (EED) develops during atrial remodeling is currently unknown. EED might enlarge the functional surface area available for wavefront propagation and has been proposed as prerequisite condition for transmural conduction and 'breakthrough' 9 . Despite its potential role of EED for AF stability only very few studies investigated simultaneous endo-epicardial activation patterns during AF 10, ${ }^{11}$. No data are currently available on the time course and the mechanisms of EED due to progressive electrical and structural remodeling of the atria.

Our aim was to examine EED as a potential mechanism leading to increasing AF stability over time. We developed techniques for quantification of EED during AF, tested the hypothesis that the increasing stability of AF correlates with the electrical dissociation between the epicardial layer and the endocardial bundle network and investigated the mechanisms underlying EED. 


\section{Methods}

\section{Model}

In this study, the goat model of persistent AF was used ${ }^{5,8,12-14}$. Three groups of goats were included ( $n=7$ in each group). The group of sham-operated animals (acute $\mathrm{AF}=\mathrm{aAF}$ ) received a right atrial endocardial pacemaker lead (Medtronic Capsurefix ${ }^{\circledR}$ ) 4 weeks prior to the open chest mapping study. In the 3 weeks AF group ( $3 \mathrm{WAF}$ ), the lead was connected to a neurostimulator (Medtronic, Itrel1 ${ }^{\circledR}$ ). In the 6 months AF group (6mAF), an additional right atrial endocardial lead connected to a pacemaker (Medtronic Kappa ${ }^{\circledR}$ ) was implanted to obtain atrial electrograms during in-vivo cardioversion experiments. All animal procedures conformed to the US National Institutes of Health guidelines and were approved by the local ethical committee for animal experiments of Maastricht University.

\section{Study Protocol}

\section{Maintenance of AF}

After two weeks of recovery from pacemaker implantation, AF was artificially maintained by repetitive $50 \mathrm{~Hz}$ burst pacing at 3 times threshold every other second. $3 \mathrm{WAF}$ goats were paced for 3 weeks. In the $6 \mathrm{mAF}$ group, pacing was performed every other second for the first 14 weeks followed by $1 \mathrm{~s} / \mathrm{min}$ for the remaining 10 weeks. No pacing was performed in the aAF group. 


\section{Cardioversion experiments}

To document increasing AF stability, all 6mAF goats underwent pharmacological cardioversion experiments with flecainide $(0.1 \mathrm{mg} / \mathrm{kg} / \mathrm{min}$, i.v. $)$. Endpoints were restoration of sinus rhythm (SR), ventricular arrhythmias, QRS prolongation of $>100 \%$, and $90 \mathrm{~min}$ infusion time. Fibrillation pacemakers were switched off at least one hour prior to the cardioversion experiments to assess the incidence of spontaneous cardioversion.

\section{Open chest experiments}

Animals were anesthetized with sufentanyl $(6 \mathrm{mg} / \mathrm{kg} / \mathrm{h})$, midazolam $(0.8 \mathrm{mg} / \mathrm{kg} / \mathrm{h})$ and pancuronium $(0.3 \mathrm{mg} / \mathrm{kg} / \mathrm{h})$. After left-sided thoracotomy, a high-density endoepicardial mapping device was positioned in the left atrium. The device was carefully closed to make contact with the endocardial bundle network and the epicardial surface. When satisfactory signals were present (sharp deflections in the absence of injury currents) the distance between the endocardial and the epicardial part of the device was fixed with a fixation screw (figure 1). A silver plate in the thoracic cavity served as indifferent electrode. Unipolar signals were recorded during AF and during atrial pacing using a custom-made 256-channel mapping amplifier (filtering bandwidth $0.1-400 \mathrm{~Hz}$, sampling rate $1 \mathrm{kHz}$, A/D resolution 16 bits). In 16/21 experiments, the mapped area was marked at the end of the experiment and photographed from the endocardial side to correlate the endocardial bundle structure with the functional data recorded. 


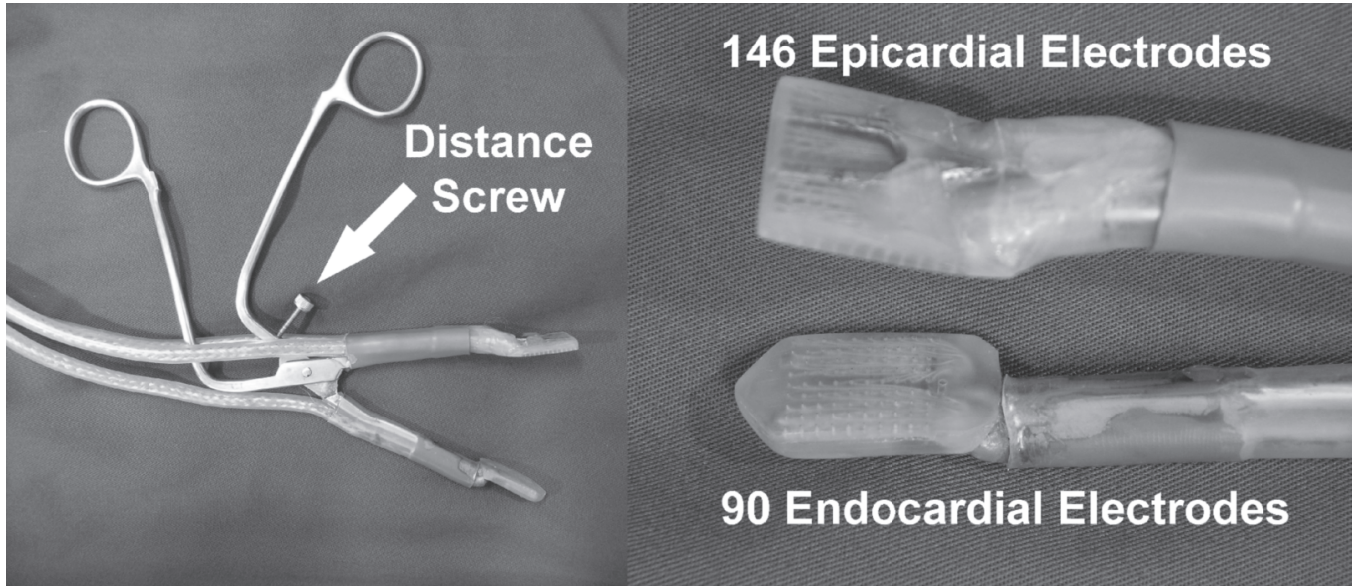

Figure 1: Clamp-like endo-epicardial mapping tool. Interelectrode distance 1.6mm, 146 epicardial (upper part) and 90 endocardial (lower part) electrodes. The (smaller) endocardial part is inserted into the atrial cavity through a small incision near the atrial appendage. A distance screw is used to measure the distance between endocardial and epicardial part of the mapping device. The 90 pairs of directly opposing electrodes are used for determination of EED.

\section{Histology}

After rapid excision, the LA was separated from the heart and parts of the LA were fixed in buffered $4 \%$ formaldehyde and imbedded in paraffin for histological evaluation. For this, tissue sections of $5 \mu \mathrm{m}$ were fixed at $56^{\circ} \mathrm{C}$ overnight, deparaffinized and rehydrated. Fibrosis was quantified on 1-2 sections per atrium (46 fields per section). To determine cardiomyocyte diameters and distances between the myocytes (intermyocyte distance), LA sections were stained with hematoxylin and eosin (H\&E). The mean cell-diameter of 30-35 transversely cut cells and respective 40-50 shortest intermyocyte distance in all four directions within one bundle were used for analysis in each goat as described earlier ${ }^{8}$. 


\section{Analysis of fibrillation electrograms}

Electrogram files recorded during $\mathrm{AF}$ (4s) were analyzed using custom-made analysis software. Local activation times were identified by maximum negative $\mathrm{dV} / \mathrm{dt}$ in each unipolar electrogram. Fibrillation maps were automatically constructed and independently edited by two researchers. Median AF cycle length (AFCL) was calculated using all fibrillation intervals of all electrodes. Conduction times were assessed by calculating the difference in activation time between each electrode and its neighbors. Conduction block was defined as conduction time between adjacent electrodes $>8 \mathrm{~ms}$ ( $>12 \mathrm{~ms}$ for oblique electrode pairs), corresponding to a conduction velocity of $<20 \mathrm{~cm} / \mathrm{s}$ which is comparable to the definition of conduction block

commonly used ${ }^{15,16}$. Apparent conduction velocity was determined by plane fitting through each activation time point and all neighboring activation times occurring within a time window of $8 \mathrm{~ms}(>12 \mathrm{~ms}$ for oblique electrode pairs, max. square of $3 \times 3$ electrodes). The resulting plane then indicated local direction (orientation of the plane) and velocity (reciprocal value of the steepness of the plane) for each local activation point. 
EED was quantified using three different methods:

1. To obtain a simple measure of endo-epicardial dyssynchrony, endo-epicardial activation time difference at each of the 90 pairs of opposing electrodes was calculated. The percentage of all endo-epicardial activation time differences above a threshold time difference was defined as "dyssynchronous" and plotted as a function of this threshold time difference. This method allowed for quantification of endoepicardial dyssynchrony independent from assumptions of cut-off values for endoepicardial conduction velocities.

2. To account for dissociation due to differences in either activation time or direction of propagation, EED was calculated from histograms of endo-epicardial differences in activation time or direction of local conduction vectors. Two distinct populations of differences in activation times or direction of local conduction vectors representing dissociated and non-dissociated activity were identified by fitting a two component Gaussian function (for activation time differences), or a Gaussian/uniform mixture distribution (for differences in direction) to the histograms. The percentage of the area under the curve formed by the dissociated component was referred to as "dissociation index" (see figure 2 for details).

3. For quantification of spatial distribution of dissociation, the analysis of activation time histograms or conduction direction difference histograms could not be used because of the limited number of data pairs at each individual electrode (between 30 and 50). Instead, all data were analyzed using dissociation thresholds derived from the activation time difference and direction difference histograms.

Two times the standard deviation of the non-dissociated components was taken as individual dissociation threshold, implying that $\sim 95 \%$ of the non-dissociated activity 
met this criterion. Activity was considered dissociated if either the activation time difference or the conduction direction difference criterion revealed dissociation for all nine closest opposing electrodes. For each electrode, the percentage of dissociation during an episode of $4 \mathrm{~s}$ was calculated and color-coded. The resulting dissociation maps were then analyzed for their local entropy to determine the degree of randomness of the distribution of dissociated and non-dissociated areas ${ }^{17}$. To test for a correlation between EED and the endocardial bundle network anatomy, bundle structures were marked manually and dissociation was calculated separately for the areas containing endocardial bundles (thick regions) and the spaces between the bundles (thin regions).

To quantify the occurrence of breakthrough, all activation times surrounded exclusively by identical or later activations - thus, representing starting points of waves in the mapping area - were identified and classified as peripheral wave (if located at the border of the mapping area) or breakthrough (if located within the mapping area). The incidence of breakthrough was then calculated as the number of wave starting points located within the mapping area per second. 


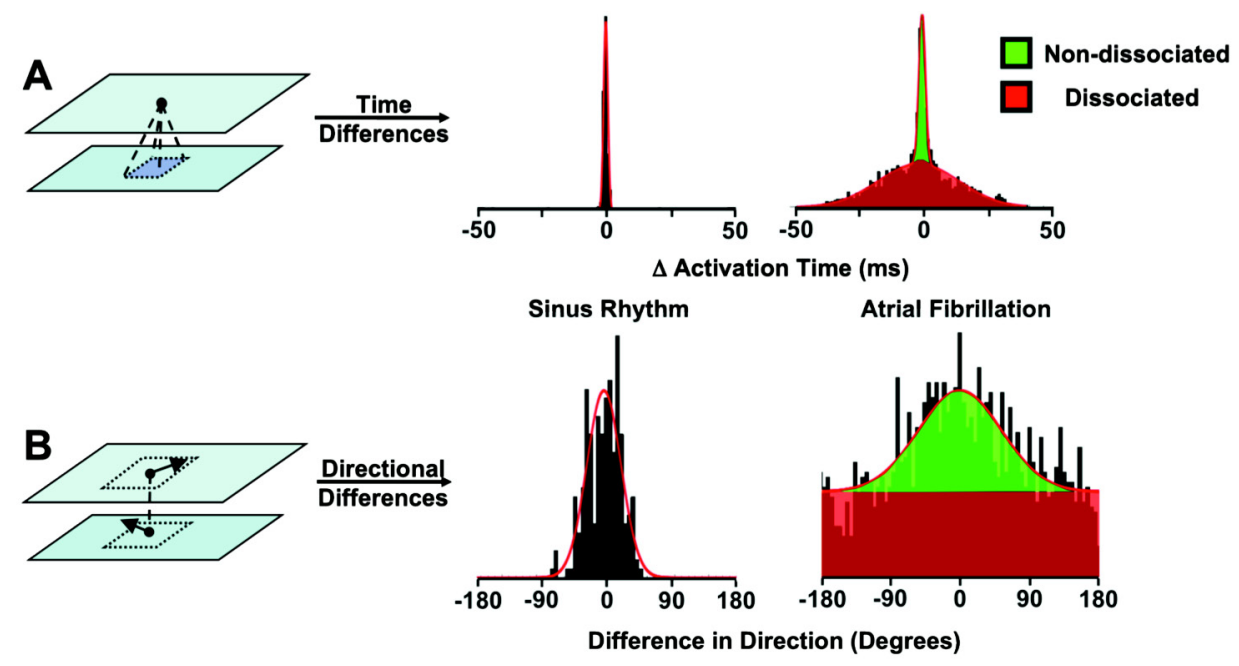

Figure 2: Curve fitting method to quantify EED:

A) Calculation of the shortest endo-epicardial activation time difference for each electrode pair. To reduce the effect of spatial noise the shortest activation time difference (normalized to the electrode distance) of the nine closest opposing electrodes was used for analysis (framed blue area on lower plane of the cartoon on the left). In the resulting histograms, the $x$-axes depict activation time differences (ms) and the $y$-axes depict the number of events. While the histogram of activation time differences during $S R$ can be fitted with a single-component Gaussian function, the histograms of activation time differences during AF are best fitted with a twocomponent Gaussian mixture distribution ${ }^{26}$. This identifies two distinct populations representing non-dissociated activations (narrow component, small standard deviation, green) and dissociated activations (broad component, large standard deviation, red). 
B) Calculation of the direction difference of corresponding local endocardial and epicardial conduction vectors (calculated based on activation times of all neighboring electrodes $=$ dotted lines). Differences in direction can be fitted with a single component Gaussian distribution during $S R$ and a Gaussian/uniform mixture distribution with the Gaussian component representing concordant endo-epicardial activations (green) and the uniform component representing discordant endoepicardial activation (red) during AF.

\section{Endo-epicardial dispersion of refractoriness and electrogram fractionation}

To test the hypothesis that EED is due to endo-epicardial dispersion of refractoriness or electrical uncoupling between the epicardial layer and the endocardial bundle network, the animals were cardioverted to SR by internal DC shock with two 6 french defibrillation catheters (Elecath, Rahway, NJ, USA) positioned in the coronary sinus and in the posterior right atrium. Defibrillation was attempted with monophasic $(5 \mathrm{~ms})$ 5J shock (Lifepak 9B, Medtronic Physio-Control Corp., Redmond, WA, USA). Energy was increased in steps of $5 \mathrm{~J}$ (max. 20J) until cardioversion was successful. ERP as well as electrogram fractionation were measured during atrial pacing. Local pacing threshold was determined separately for the endocardial and the epicardial pacing site and bipolar pacing $(1.6 \mathrm{~mm}$ interelectrode distance) was performed from the centers of the electrode arrays at $250 \mathrm{~ms}$ and $400 \mathrm{~ms}$ basic cycle length (CL) at four times (current)-threshold. Only sites at which both, the endocardial and epicardial threshold was low $(<2 \mathrm{~mA})$ were used for analysis.

The longest S1-S2 interval (2ms incremental steps) not resulting in a propagated response was taken as local ERP. Epicardial electrogram fractionation was 
determined during rapid pacing $(C L=200 \mathrm{~ms})$ from a pacing site outside the mapping array. Atrial complexes showing two separate deflections, with a minimum amplitude of $25 \%$ of the larger deflection occurring within a time window of 2 to $15 \mathrm{~ms}$ were taken as "short double potentials" ${ }^{18}$. "Complex fractionated potentials" were defined as atrial complexes with 3 or more negative deflections.

\section{Statistical analysis}

All data are expressed as mean $\pm S D$. Significance of differences in means between aAF, 3WAF, and 6mAF groups was calculated using a one way ANOVA with Newman-Keuls post-test and a paired Student t-test for significance of differences in means between the endocardial and the epicardial layer in the same specimen. $\mathrm{P}$ values $<0.05$ were considered significant. 


\section{Results}

\section{Cardioversion experiments}

The cardioversion experiments in the $6 \mathrm{mAF}$ group documented increasing $\mathrm{AF}$ stability over time. AF was persistent in all 7 goats undergoing cardioversion experiments after 2 weeks of AF. At that time point, pharmacological cardioversion to SR was successful in 6 out of 7 goats (85\%). The success rate declined to $3 / 7(43 \%)$ after 6 weeks, $1 / 7(14 \%)$ after 10 weeks and 0/7 (0\%) after 14 weeks. No animal that cardioverted successfully had failed to cardiovert at an earlier time point. Baseline AFCL did not differ significantly between 2 weeks and 14 weeks of AF $(116 \pm 24 \mathrm{~ms}$ vs. $117 \pm 21 \mathrm{~ms}$, n.s.).

\section{Enhanced heterogeneity of conduction in both the epicardial layer and the endocardial bundle network}

Table 1 summarizes the basic electrophysiological parameters of AF found in the 3 groups of animals. During open chest experiments, the mean AFCL was significantly longer in the aAF group compared to the $3 \mathrm{WAF}$ group $(p<0.01)$. No further shortening of the AFCL was found in the $6 \mathrm{mAF}$ group. The median $\left(\mathrm{P}_{50}\right)$ of normalized phase differences (in $\mathrm{ms} / \mathrm{mm}$ ) as well as the heterogeneity index ( $\mathrm{P}_{95}$ $\left.P_{5}\right) / P_{50}$ ) were significantly larger in the $6 \mathrm{mAF}$ and the $3 \mathrm{wAF}$ group compared to the aAF group $(p<0.05)$. Incidence of conduction block between neighboring electrodes was highest in the $6 \mathrm{mAF}$ and lowest in the aAF group $(p<0.05)$. There was a trend towards more conduction disturbances endocardially, but no significant differences in $\mathrm{AFCl}$, heterogeneity indices, or incidence of conduction block between the epicardial layer and the endocardial bundle network. 


\begin{tabular}{|c|c|c|c|c|}
\hline & & Acute & 3 weeks & 6 months \\
\hline \multicolumn{2}{|c|}{ AFCL (ms) } & $141 \pm 15$ & $121 \pm 10^{*}$ & $120 \pm 5^{*}$ \\
\hline \multicolumn{2}{|c|}{$P_{50}$ phase differences $(\mathrm{ms} / \mathrm{mm})$} & $0.7 \pm 0.3$ & $1.0 \pm 0.4^{*}$ & $1.4 \pm 0.2^{*}, \dagger$ \\
\hline \multirow{2}{*}{$\begin{array}{c}\mathrm{P}_{95}-\mathrm{P}_{5} / \mathrm{P}_{50} \\
\text { phase differences }\end{array}$} & Endocardial & $9.9 \pm 3.4$ & $14.6 \pm 4.7^{*}$ & $13.0 \pm 1.5^{*}$ \\
\hline & Epicardial & $7.4 \pm 2.4$ & $10.7 \pm 3.2^{*}$ & $11.2 \pm 1.8^{*}$ \\
\hline \multirow{2}{*}{$\begin{array}{l}\text { Incidence of block } \\
\text { (\% of activations) }\end{array}$} & Endocardial & $8.0 \pm 3.8$ & $15.7 \pm 3.0^{*}$ & $17.4 \pm 4.7^{*}, \dagger$ \\
\hline & Epicardial & $6.0 \pm 2.8$ & $11.3 \pm 4.9^{*}$ & $16.4 \pm 2.9^{*}, \dagger$ \\
\hline
\end{tabular}

Table 1: Basic electrophysiological parameters

Significant electrical remodeling reflected by AFCL decrease (Acute-AF vs. 3weeks$A F)$, increasing conduction times, heterogeneity index $\left(P_{95}-P_{5} / P_{50}\right.$ of conduction times) and increased incidence of block. ${ }^{*}=$ significant vs. Acute-AF, $\dagger=$ significant vs. 3weeks-AF 


\section{Endo-epicardial dissociation during AF}

Figure 3 illustrates EED in a goat after 3 weeks of AF.

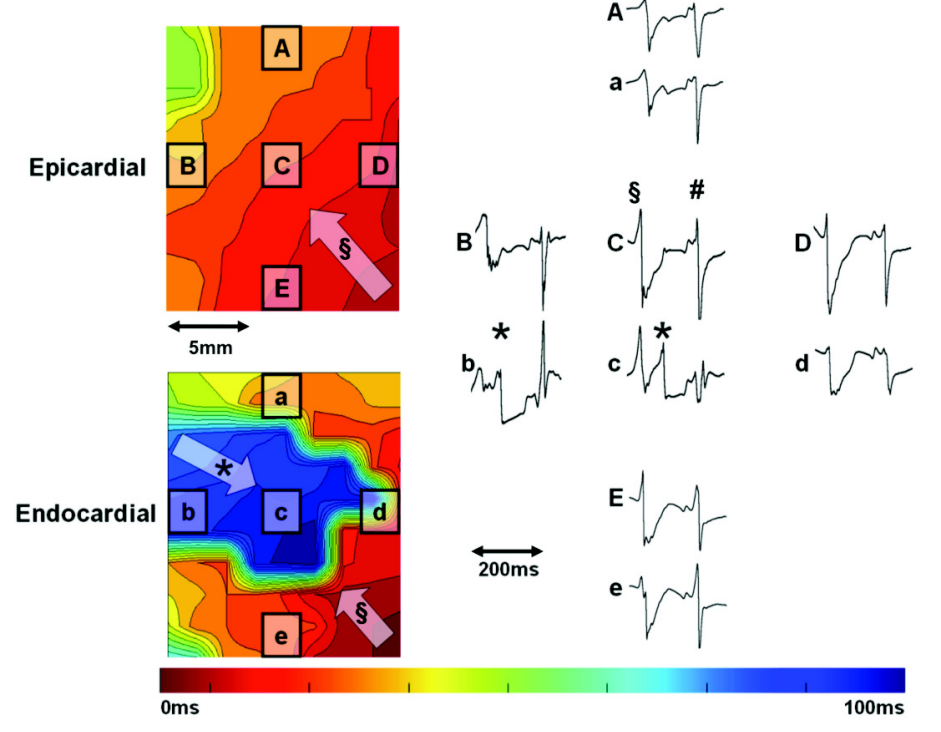

Figure 3: Endo-epicardial isochronal map (3wAF) of two exactly corresponding areas of the epicardial and endocardial layer. Time between isochrones $5 \mathrm{~ms}$, early activations color coded in red, late in blue. Five corresponding

electrogram tracings (epicardial $A-E$, endocardial a-e) are displayed on the right. $A$ broad fibrillation wave enters the mapping area from the lower right corner, where it activates both the epicardial as well as the endocardial layer. The electrograms $b$ and c show two deflections between 50 and 100ms (marked by "§" and "*"). The deflections marked with "," represent a wave which enters the mapping area from the left and which exclusively propagates within the endocardial layer (blue wave in isochronal map). The final deflections in the electrogram tracings represent the next wave, which again activates both layers of the atrial wall (not displayed in the isochronal map, electrograms marked with \#). 
For all goats, each of the three different quantification methods of EED showed an increase of dissociation with AF duration.

1. Dyssynchrony of endo-epicardial activation (figure 4).

For any threshold of endo-epicardial activation time differences above 0ms, the percentage of "dyssynchronous" activation time differences was highest in the 6mAF group and lowest in the aAF group. Differences in dyssynchrony between groups were most pronounced at a threshold activation time difference of $10 \mathrm{~ms}$. At this threshold value, the percentage of dyssynchronous activity was $50.3 \pm 10.4 \%$ in the $6 \mathrm{mAF}$ group, $30.8 \pm 10.3 \%$ in the $3 \mathrm{WAF}$ group, and $15.7 \pm 4.4 \%$ in the aAF group $(p<0.01)$.

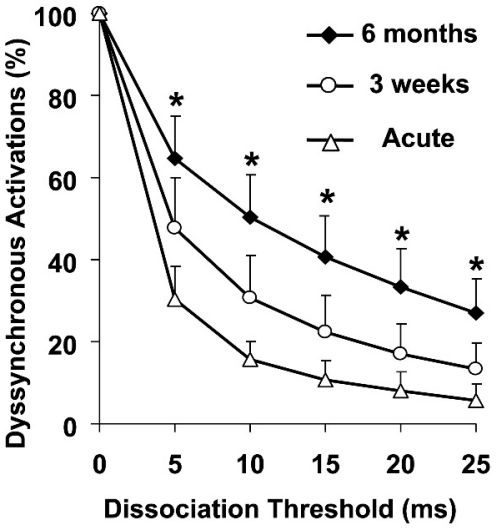

Figure 4: Percentage dyssynchronous activity as a function of the endo-epicardial activation time difference threshold.

Y-axis: Percentage of activation time differences above various threshold activation time differences.

$X$-axis: activation time difference thresholds in $m s .{ }^{*}=p<0.01 \mathrm{vs} .3 w A F$ and $a A F$. 
2. Histograms of endo-epicardial differences in activation time and direction of local conduction vectors.

The histograms of activation time differences and differences in direction of conduction vectors showed two distinct components with different behavior. Calculations of histograms after artificial shift of endocardial against epicardial activation times suggest that the narrow component reflects a temporal or directional relation between electrical activity in the epicardial layer and the endocardial bundle network (i.e. non-dissociated electrical activity). The wide or uniform components reflect lack of such an interrelation suggesting that epicardial layer and endocardial bundle network are activated independently.

To test for this hypothesis the original epicardial electrograms were shifted versus the endocardial electrograms in time steps of $10 \mathrm{~ms}$. After each time step the histograms were recalculated. This revealed that, while the 'synchronous' narrow component clearly is shifted in agreement with the time step used, the dissociated, wide component remains unchanged and centered (example depicted in figure 5, upper graphs). Computing this shift for all 21 datasets resulted in the graph depicted in figure 5 online lower graph. The mean of the dissociated component remained near $0 \mathrm{~ms}$, in agreement with the assumption of a random distribution of endoepicardial activation time differences in case fibrillation waves propagate independently in the epicardial layer and the endocardial bundle network. In contrast, the mean of the non-dissociated component is shifted in time, as one would expect from the time step applied. This suggests that the non-dissociated component reflects the existence of a temporal interrelation between the electrical activity of the epicardial layer and the endocardial bundle network as expected during transmural 
conduction or within fibrillation waves that simultaneously activate the epicardial layer and the endocardial bundle network. We conclude from this observation that the narrow component reflects non-dissociated electrical activity with functional interaction of the electrical activity in the epicardial layer and the endocardial bundle network. In contrast, the wide component reflects electrical activity without any functional interrelation and therefore is regarded as dissociated activity.
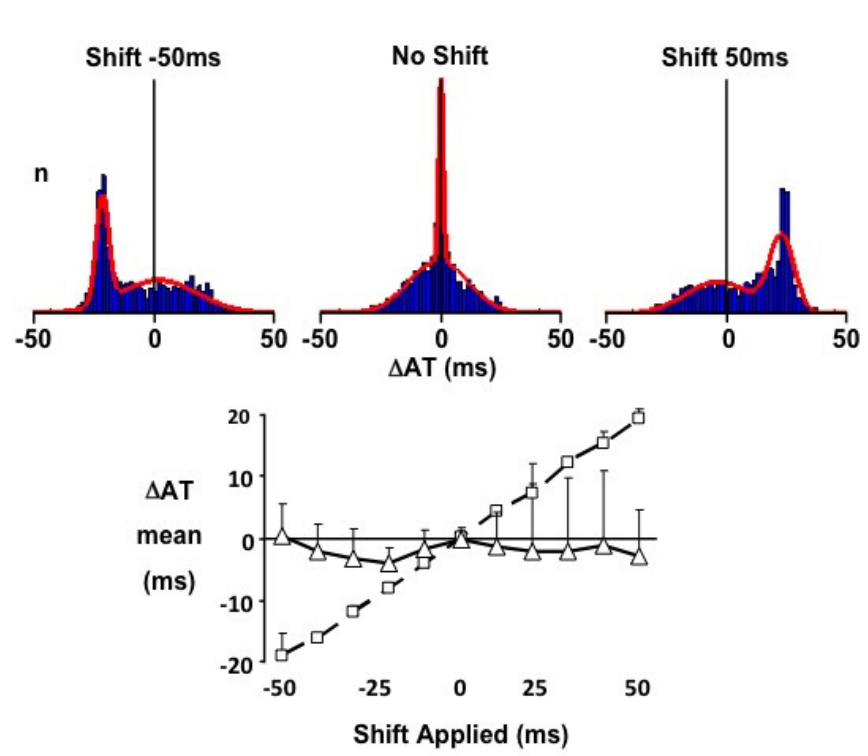

$\triangle-$ Dissociated Component $\quad \square-$ Non-dissociated Component

Figure 5:

Upper panel: Original epicardial signals shifted versus endocardial signals by 50 or $-50 m s$, illustrating that the narrow component (representing the nondissociated activations) shifted according to the time shift while the broad component (representing the dissociated activations) remained unchanged and centered.

Lower panel: Analysis including all activations of all 21 files depicting the means of the dissociated component (triangle) and the non-dissociated component (square) for shifts (Endocardial versus corresponding epicardial signals) in steps of 10ms. $X$-axis: time shift added to the endocardial activation times in ms, $y$-axis: mean of the two components (ms). 
Note that $y$-values are given as activation times normalized for the endo-epicardial electrode distance $(2.7 \pm 0.4 \mathrm{~mm})$ which explains that a $50 \mathrm{~ms}$ time step results in a time shift of the non-dissociated component of $18.5 \mathrm{~ms}$.

Figure 6 shows histograms of activation time differences (panel A) and histograms of differences in direction of propagation (panel B) for all 21 goats. During acute AF, the index of EED was $17 \pm 7 \%$ based on the activation time difference criterion and $17 \pm 10 \%$ based on the direction difference criterion. In the $3 \mathrm{WAF}$ group, the dissociation index increased significantly to $39 \pm 17 \%$ (time criterion) and $53 \pm 18 \%$ (direction criterion) ( $p<0.05$ compared to aAF). After 6 months of $A F$, as much as $68 \pm 13 \%$ (time criterion) and $72 \pm 14 \%$ (direction criterion) of the electrical activity was dissociated between the epicardial layer and the endocardial bundle network $(p<0.05$ compared to aAF and $3 \mathrm{WAF})$.
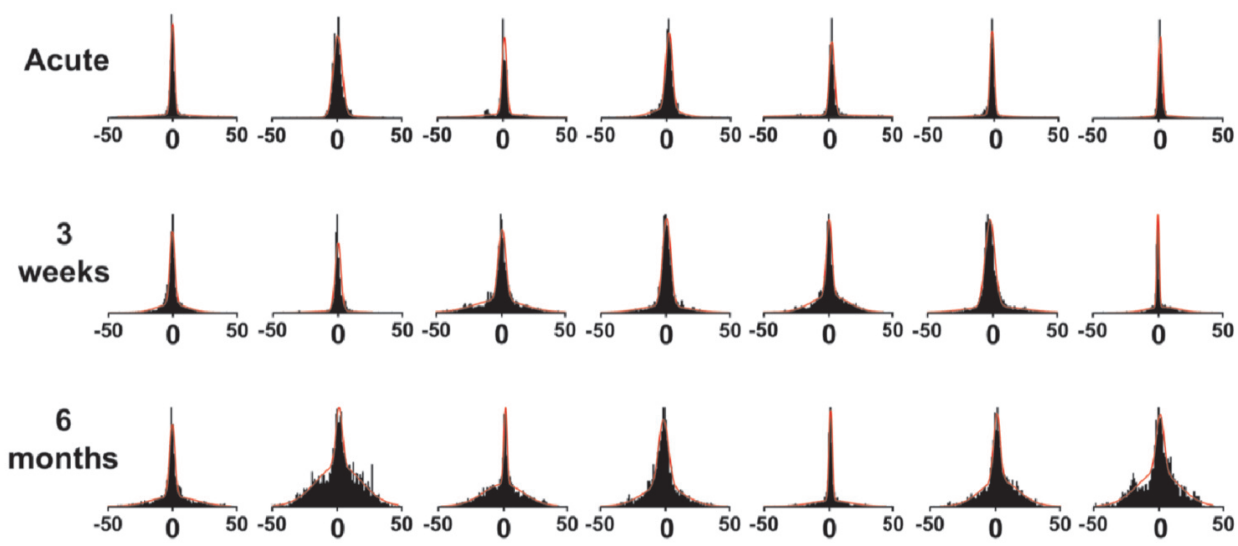

Figure $6 \mathrm{~A}$ :

Endo-epicardial activation time difference histograms of all 21 individual goats. 
$X$-axes depict activation time differences between corresponding endo-epicardial electrode pairs in $\mathrm{ms}, y$-axes depict number of events (normalized to allow comparison of groups). Red fitted lines represent the curves resulting from the twocomponent Gaussian mixture distribution. The relative contribution of the dissociated component (low peak, wide SD) increases with AF duration (Upper to lower row).
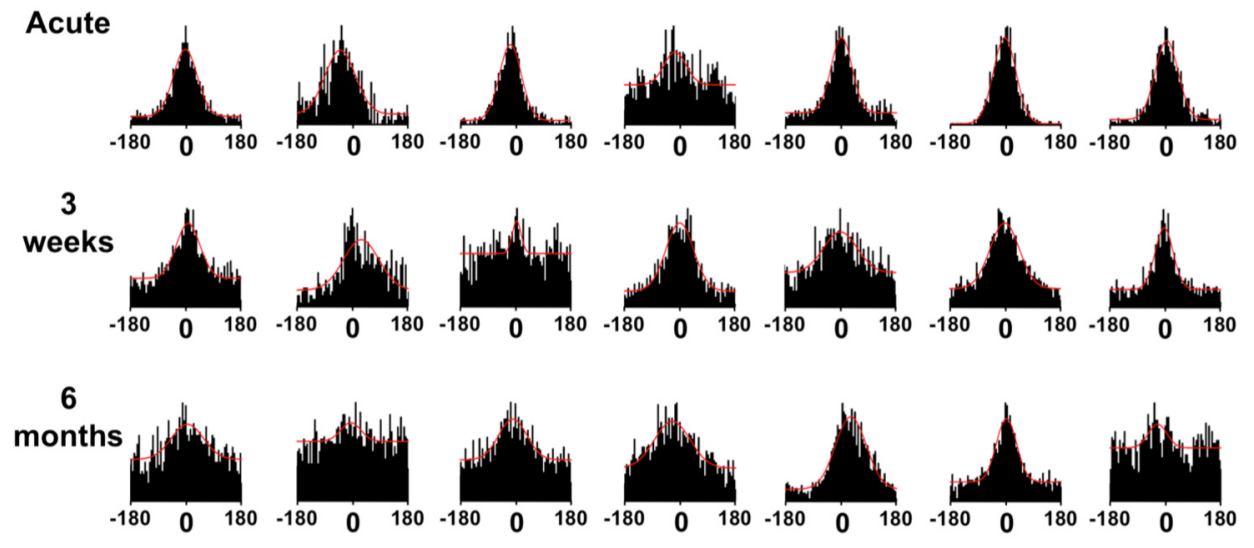

Figure 6 B:

Endo-epicardial difference in directions of local conduction vectors histograms of all 21 individual goats.

$X$-axes depict difference in direction (-180 to +180 degrees), $y$-axes depict number of events (normalized to allow comparison of groups). Red fitted lines represent the curves resulting from the Gaussian/uniform mixture distribution. The relative contribution of the dissociated component (uniform distribution) increases with AF duration. 
Interestingly, mean endo-epicardial activation time difference was close to 0ms and not different in all three groups (aAF: $0.87 \pm 3.04 \mathrm{~ms}, 3 \mathrm{WAF}:-0.82 \pm 4.71 \mathrm{~ms}, 6 \mathrm{mAF}$ : $0.14 \pm 1.23 \mathrm{~ms}, \mathrm{~ns})$, arguing against preferential conduction from the epicardial layer to the endocardial bundles network or vice versa.

3. Spatial distribution of dissociation.

Spatial analysis revealed that the degree of dissociation was not uniformly distributed over the mapping area but that there were areas with higher and lower degrees of EED (figure 7, upper and left lower panel). To test for randomness we calculated the entropy value for spatial distribution of dissociation (1=random distribution, $0=$ no randomness). There was no difference in entropy between groups (aAF: $0.63 \pm 0.15,3 w A F: 0.54 \pm 0.09,6 m A F: 0.55 \pm 0.12$, all $n=7, n s$ ).

In 16/21 experiments, we compared the dissociation map with the underlying endocardial bundle structure (figure 7, lower right panel). On average for all groups, EED was higher in thick areas of the atrial wall (thick: $49.3 \pm 21.4 \%$, thin: $42.2 \pm 19.0 \%$, $\mathrm{n}=16, \mathrm{p}<0.05$ ). The difference was similar in the aAF group (thick: $29.7 \pm 10.8 \%$, thin: $23.7 \pm 11.1 \%, n=7, p<0.01$ ) compared to the $6 \mathrm{mAF}$ group (thick: $67.8 \pm 12.5 \%$, thin: $59.0 \pm 11.4 \%, n=7, p<0.01)$. Endo-epicardial electrode distance decreased from $3.0 \pm 0.4 \mathrm{~mm}$ in the aAF group to $2.5 \pm 0.3 \mathrm{~mm}$ in the $6 \mathrm{mAF}$ group, reflecting moderate thinning of the dilated atria during 6 months of $A F(p<0.05)$. 

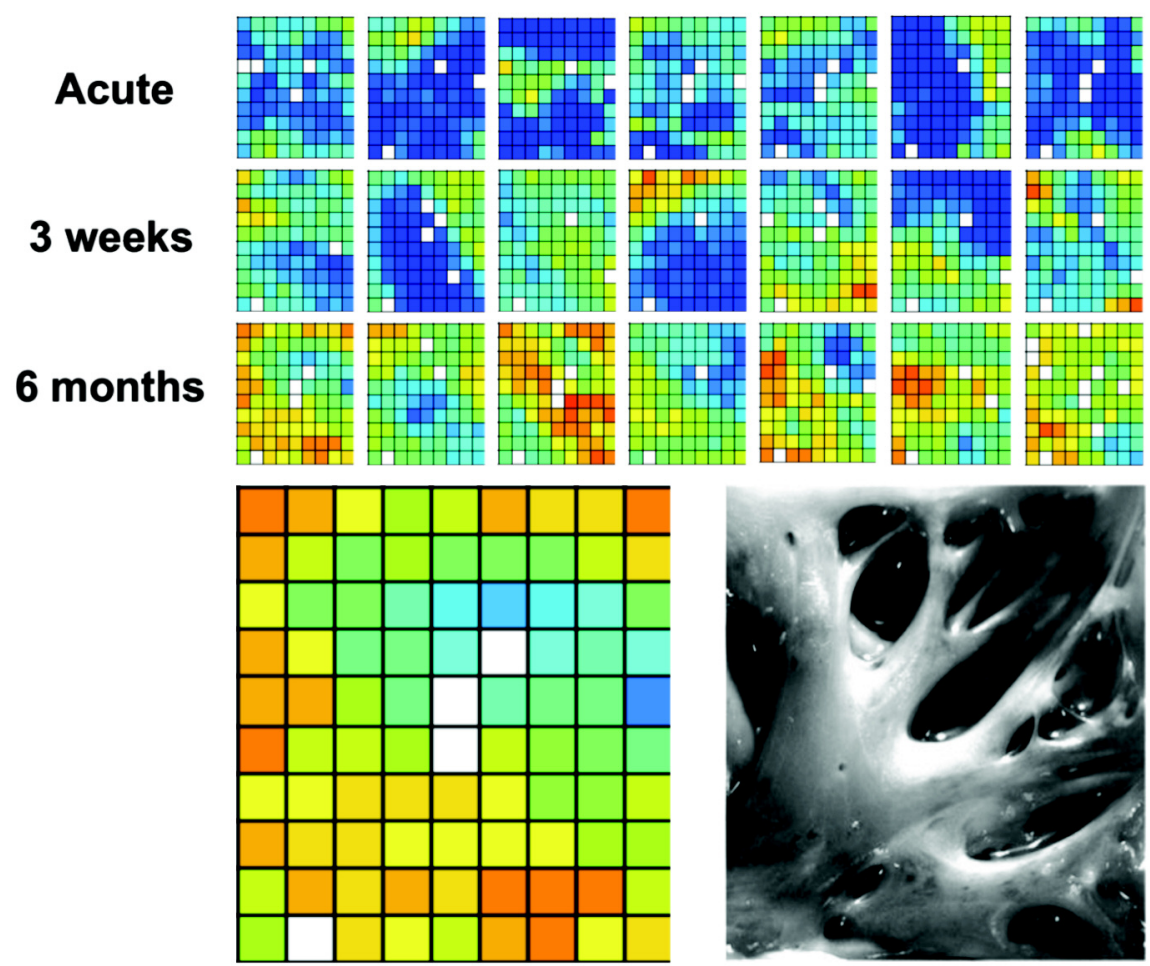

\section{Dissociated}

Non-dissociated

Figure 7: Upper panel: Dissociation maps of all 21 goats indicating spatial distribution of dissociation in each individual goat.

Blue color indicates little to no dissociation, red color indicates pronounced dissociation. Color changes toward yellow and red indicate that dissociation increases with AF duration. Inhomogeneous patterns of spatial distribution of dissociation within individual maps are apparent, indicating an influence of underlying atrial structure on the degree of dissociation.

Lower left: Individual dissociation map of a goat after 6 months AF. Lower right: Corresponding endocardial bundle structure. 


\section{Incidence of breakthrough}

The proportion of wave starting points located within the mapping area increased significantly from $0.8 \pm 0.6 / \mathrm{s}$ in the aAF group to $6.2 \pm 3.1 / \mathrm{s}$ in the $3 \mathrm{WAF}$ group and $13.6 \pm 5.3 / \mathrm{s}$ in the $6 \mathrm{mAF}$ group $(p<0.001)$. Both epicardial and endocardial breakthroughs were included in the analysis.

Endo-epicardial dispersion of refractoriness and fractionation of atrial electrograms

All animals in persistent $A F$ (3WAF and $6 \mathrm{mAF}$ group) could be successfully cardioverted by internal DC shock. No apparent differences for pacing thresholds were noticed between the endo- and epicardium. Endocardial ERPs were significantly longer compared to the corresponding epicardial ERPs at both 250 and $400 \mathrm{~ms}$ in the aAF group. After 6 months of AF, there was no statistical significant difference anymore between the epicardial and the endocardial ERPs (table 2). Importantly, both the incidence of short double potentials and the fractionation intervals were larger after 3 weeks of AF compared to aAF and further increased after 6 month of AF (figure 8, table 2). Complex fractionated electrograms (3 or more deflections) were observed in $6 \mathrm{~m}-\mathrm{AF}$ goats only. Of note, one of the epicardial deflections coincided $(\Delta t<2 \mathrm{~ms})$ with the endocardial deflection in $87 \%$ of all short double potentials. 
Acute 3 weeks 6 months

\begin{tabular}{lccc}
\hline$\Delta$ ERP (endo-epicardial, ms) & $21.8 \pm 18$ & $17.3 \pm 8^{*}$ & $3.7 \pm 16^{*}, \dagger$ \\
\hline Fractionated electrograms (\%) & $9.4 \pm 1.9$ & $12.8 \pm 2.7^{*}$ & $18.6 \pm 0.6^{*}, \dagger$ \\
\hline Separation interval (ms) & $4.8 \pm 0.5$ & $6.1 \pm 0.5^{*}$ & $9.4 \pm 0.6^{*}, \dagger$ \\
\hline
\end{tabular}

Table 2:

Endocardial ERPs were longer compared to epicardial only at baseline $(p<0.001)$, but not after 6 months AF. Fractionation and separation interval during rapid atrial pacing increased significantly with AF duration.

* = significant vs. Acute-AF, $\dagger=$ significant vs. 3weeks-AF

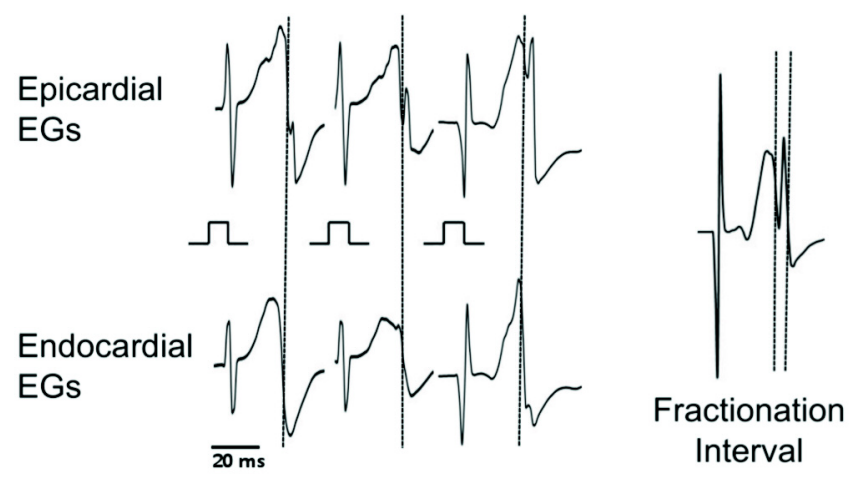

Figure 8: Left: Examples of epicardial short double potentials during rapid atrial pacing. In each example one of the two epicardial deflections after the pacing artifact coincides with an endocardial activation and represents a farfield signal (dotted lines). Right: Time interval between first and second deflection was determined and taken as fractionation interval (between dotted lines). EGs = Electrograms 


\section{Histology}

In the $6 \mathrm{mAF}$ group, myocyte diameters were significantly larger than in the $3 \mathrm{WAF}$ group and the aAF group $(10.9 \pm 0.6 \mathrm{~mm}$ aAF, $13.6 \pm 1.0 \mathrm{~mm} 3 \mathrm{WAF}, 17.0 \pm 1.6 \mathrm{~mm}$ $6 \mathrm{mAF}, \mathrm{p}<0.056 \mathrm{mAF}$ vs. $3 \mathrm{WAF}$ and $\mathrm{aAF}$ ). Intermyocyte distances as measure of extracellular matrix were larger in the $6 \mathrm{mAF}$ group $(6.8 \pm 1.6 \mu \mathrm{m})$ than in the $3 \mathrm{WAF}$ group $(2.3 \pm 0.3 \mu \mathrm{m}, \mathrm{p}<0.05)$ and the aAF group $(1.8 \pm 0.2 \mu \mathrm{m}, \mathrm{p}<0.05)$. No significant differences were found for these parameters between the aAF and the 3wAF group (figure 9).

Acute AF

\section{3 weeks AF}
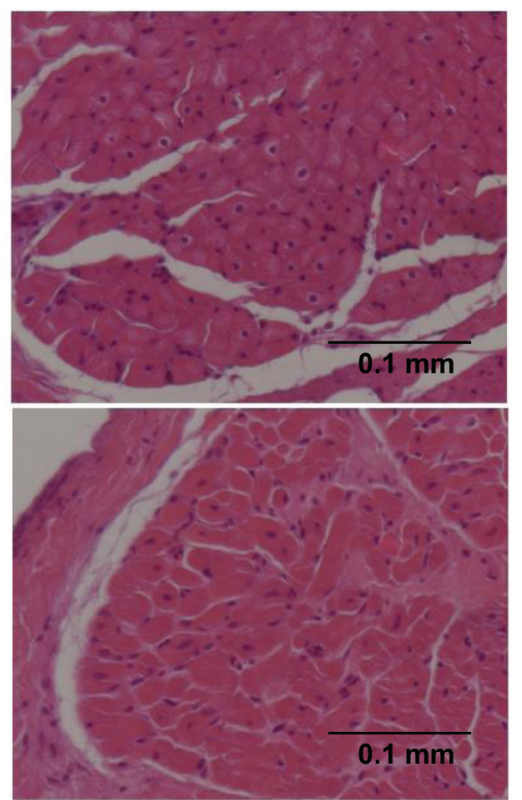

\section{6 months AF}

Figure 9: Representative examples of left atrial tissue (H\&E stain, $\quad 800 x$ magnification) of the aAF, $3 W A F$ and $6 \mathrm{mAF}$ group. Cardiomyocyte diameter and intermyocyte distance increase with AF duration. 


\section{Discussion}

Local heterogeneities in conduction and conduction block due to increasing electrical uncoupling between muscle bundles have been suggested to contribute to increasing complexity and stability of AF over time. Dissociation of electrical activity between the epicardial layer and the endocardial bundle network has been postulated and was seen as a prerequisite for epicardial breakthrough events ${ }^{9,15,19}$, which more frequently occur in complex substrates for $\mathrm{AF}^{20}$. However, data directly addressing the existence of EED are sparse 10, 11, 19, and its development during structural remodeling has not been studied so far. Our study confirms the existence of electrical dissociation between the epicardial layer and the endocardial bundle network during AF. EED was reflected both by dyssynchronous activation of the epicardial layer and the endocardial bundle network and by differences in direction of propagation. We demonstrate that during the development of a substrate of $A F$, the complexity of conduction patterns is associated with a pronounced increase in EED. Importantly, this process still continues after electrical remodeling has occurred. EED is not randomly distributed but more pronounced in thicker regions of the atrial wall. The main mechanism resulting in EED during $A F$ is not related to transmural dispersion of refractoriness but to progressive electrical uncoupling between the epicardial layer and the endocardial bundle network. 


\section{Comparison with previous studies}

Schuessler et al. were the first to directly demonstrate endo-epicardial dyssynchrony of activation during acetylcholine-induced $\mathrm{AF}$ in canine right atria ${ }^{10}$. They demonstrated that during acute $\mathrm{AF}$, endo-epicardial activation time differences became larger, particularly in thicker areas of the atrial wall and in areas with several layers with different bundle orientations. Our study extends these findings to changes occurring as a consequence of AF-induced structural remodeling and an analysis of EED based on differences in propagation direction. We confirmed that in the left atrium, EED is more pronounced in thicker parts of the atrium than in thinner parts. However, with increasing duration of AF the thickness of the atrial wall declined while EED significantly increased. This suggests a more important role of AF induced structural remodeling than of the absolute thickness of the atrial wall for the occurrence of EED ${ }^{14}$.

Epicardial mapping studies in patients with persistent AF have demonstrated that the incidence of epicardial breakthrough, i.e. transmural conduction of a wavefront from the opposing side of the atrial wall, increases with increasing duration of $\mathrm{AF}$ and increasing complexity of the conduction pattern ${ }^{7,20}$. The present study supports this finding and offers an explanation for the increased incidence of epicardial breakthroughs. It demonstrates that during the development of the substrate for AF electrical dissociation between the endocardial bundle network and the epicardial layer progressively increases and because of this, fibrillatory conduction gradually becomes a more 3-dimensional process. As EED is the prerequisite condition for breakthroughs to occur, enhanced EED might underlie the high incidence of breakthrough in a complex substrate for AF. 
Recently, Everett et al. compared virtual endocardial electrograms (Ensite ${ }^{\circledR}$ ) with epicardial direct contact electrograms in four different canine models (congestive heart failure, mitral regurgitation, rapid atrial pacing, and methylcholine-induced $A F)^{11}$. Their analysis focused on differences in electrogram morphology and revealed the largest endo- to epicardial differences in the rapid atrial pacing model. Dominant frequencies of electrical activation were lower in the endocardium than in the epicardium. However, the study by Everett et al did not show whether the endoepicardial differences in electrogram morphology, recorded with two different techniques, indeed resulted in differences in activation times and propagation direction of fibrillation wavefronts. In our study, direct contact unipolar electrograms were used for analysis of activation times on both sides of the atrial wall using a tool with precisely aligned electrode pairs. Our measurements did not show different activation rates in the endocardium versus epicardium, but a comparable AFCL in both layers of the atrial wall. The electrophysiological changes in the rapid atrial pacing group of Everett et al. and the 3 weeks persistent AF group of the present study are likely to be comparable. In both models significant endo-epicardial dissociation of electrical activity occurs. In models of structural remodeling of the atria, however, like for example congestive heart failure or mitral valve regurgitation, macro-reentry often underlies AF. In the study by Everett et al., indeed a lower incidence of conduction block and less electrical dissociation was observed in the mitral valve regurgitation model and the heart failure model compared to the RAP model. This might explain why in these models only little dissociation between the epicardial and endocardial layers was observed. In contrast, in goats with 6 months of $\mathrm{AF}$, the combination of electrical remodeling and significant extracellular matrix 
alterations might underlie the pronounced electrical dissociation within and between the endo- and epicardial layer of the atrial wall.

\section{Potential mechanisms leading to endo-epicardial dissociation}

Structural changes in the atria and gap junction remodeling during the course of $\mathrm{AF}$ have been extensively studied in our model ${ }^{13}, 14,21-23$. Van der Velden et al. demonstrated a heterogeneous reduction in $\mathrm{Cx} 40$ (but not $\mathrm{Cx} 43$ ) over a time course of several months ${ }^{23}$. After 9 to 23 weeks of AF, Ausma described that up to $92 \%$ of myocytes showed marked ultrastructural changes and a marked increase in myocyte size by $195 \%^{13}$. In this study, extracellular matrix fraction per myocyte was significantly increased both in right and in left atria after 4 months of AF. In the present study, histological analysis confirmed significant myocyte hypertrophy and more importantly - a pronounced increase in intermyocyte distances due to enhanced extracellular matrix formation. Spach et al. have shown that in aging atria, this pattern of interstitial fibrosis may lead to disruption of electrical side-to-side connections, impaired conduction and the occurrence of fractionated electrograms ${ }^{6}$. In our study, we hypothesize that electrical side-to-side connections are not only disrupted within the epicardial layer but also between the epicardial layer and the endocardial bundle network. To support this hypothesis, we demonstrated enhanced fractionation of electrograms in broad wavefronts during atrial pacing with increasing duration of AF. One of the epicardial deflections coincided with a local endocardial deflection in most cases, suggesting that fractionation was due to a slight dyssynchrony in the activation of the epicardial layer and the endocardial bundle network. Enhanced electrogram fractionation due to EED was associated with longer 
AF duration and increasing local heterogeneity, suggesting that it contributes to the complexity of the substrate and the stability of the arrhythmia.

We also addressed the question whether endo-epicardial dispersion of refractoriness might have contributed to EED during AF. Indeed we found that at baseline, endocardial ERPs were longer than epicardial ERPs (table 2). The transmural dispersion of refractoriness was smaller in the 3WAF group and after 6 months no endo-epicardial dispersion of refractoriness was detectable anymore. Wang et al. studied endo- and epicardial action potential durations (APD) in superfused atrial tissue of guinea pigs ${ }^{24}$. These authors demonstrated longer APDs in the endocardial than the epicardial layer presumably due to larger transient outward currents $\left(I_{t o}\right)$ in the epicardium. Downregulation of $\mathrm{I}_{\text {to }}$ during $\mathrm{AF}^{25}$ might have contributed to the reduction of transmural ERP dispersion in the atria of animals in the 3WAF and the $6 \mathrm{mAF}$ groups. Regardless the underlying mechanism of the endo-epicardial dispersion of refractoriness at baseline, we conclude that endo-epicardial differences in refractoriness do not play a role in enhancement of EED with increasing duration of $A F$. 


\section{Contribution of endo-epicardial dissociation to the AF substrate}

EED could lead to increased AF stability by different mechanisms. First, it increases the functional area available for wavefront propagation. EED thereby enables the coexistence of more wavelets. Secondly, EED forms the prerequisite for transmural conduction. The more the epicardial layer and the endocardial bundle network are activated out of phase, the more likely a fibrillation wave will encounter excitable tissue on the other side of the atrial wall and transmurally propagate from the endocardial bundle network to the epicardial layer or vice versa. Each breakthrough might then develop into an independent fibrillation wave contributing to the fibrillation process. In this sense, breakthroughs represent additional reentry points for reactivation of areas that just regained excitability.

\section{Potential limitations}

The mapping tool was designed for endo-epicardial mapping of the left atrial free wall in a heart exposed by left-sided thoracotomy. Whether our findings are applicable for other atrial regions, with different bundle anatomy, warrants further investigation. 


\section{Conclusion}

AF stability progressively increases with AF duration even after electrical remodeling has occurred. During the first 6 months increasing AF stability is associated with an increase in EED. Not endo-epicardial dispersion of refractoriness, but progressive electrical uncoupling between the epicardial layer and the endocardial bundle network is the underlying mechanism. EED leads to AF stabilization by providing more functional surface for fibrillation wavelets to coexist and represents a prerequisite condition for transmural conduction with reactivation of atrial myocardium in the opposing layer of the atrial wall. The mechanisms described are likely to contribute to the progressive stability of AF over time by increasing the complexity of three dimensional fibrillation patterns. 


\section{References}

1. Kopecky SL, Gersh BJ, McGoon MD, Whisnant JP, Holmes DR, Jr., Ilstrup $\mathrm{DM}$ et al. The natural history of lone atrial fibrillation. A population-based study over three decades. N Engl J Med 1987;317:669-674.

2. Zrenner B, Ndrepepa G, Karch MR, Schneider MA, Schreieck J, Schomig A et al. Electrophysiologic characteristics of paroxysmal and chronic atrial fibrillation in human right atrium. J Am Coll Cardiol 2001;38:1143-1149.

3. Allessie MA, Lammers W, Bonke F, Hollen J. Experimental evaluation of Moe's multiple wavelet hypothesis of atrial fibrillation In: Zipes D, Jalife J, eds. Cardiac Electrophysiology and arrhythmias. New York: Grune \& Stratton, 1985:265-275.

4. Allessie MA, Ausma J, Schotten U. Electrical, contractile and structural remodeling during atrial fibrillation. Cardiovasc Res 2002;54:230-246.

5. Wijffels MC, Kirchhof CJ, Dorland R, Allessie MA. Atrial fibrillation begets atrial fibrillation. A study in awake chronically instrumented goats. Circulation 1995;92:1954-1968.

6. Spach MS, Dolber PC. Relating extracellular potentials and their derivatives to anisotropic propagation at a microscopic level in human cardiac muscle. Evidence for electrical uncoupling of side-to-side fiber connections with increasing age. Circ Res 1986;58:356-371.

7. Allessie MA, de Groot NM, Houben RP, Schotten U, Boersma E, Smeets JL et al. The ElectroPathological Substrate of Longstanding Persistent Atrial Fibrillation in Patients with Structural Heart Disease: Longitudinal Dissociation. Circ Arrhythm Electrophysiol;

8. Verheule S, Tuyls E, Van Hunnik A, Kuiper M, Schotten U, Allessie MA. Fibrillatory conduction in the atrial free walls of goats in persistent and permanent atrial fibrillation. Circ Arrhythm Electrophysiol 2010;

9. Konings KT, Kirchhof CJ, Smeets JR, Wellens HJ, Penn OC, Allessie MA. High-density mapping of electrically induced atrial fibrillation in humans. Circulation 1994;89:1665-1680.

10. Schuessler RB, Kawamoto T, Hand DE, Mitsuno M, Bromberg BI, Cox JL et al. Simultaneous epicardial and endocardial activation sequence mapping in the isolated canine right atrium. Circulation 1993;88:250-263. 
11. Everett TH, Wilson EE, Hulley GS, Olgin JE. Transmural characteristics of atrial fibrillation in canine models of structural and electrical atrial remodeling assessed by simultaneous epicardial and endocardial mapping. Heart Rhythm 2010;7:506-517.

12. de Haan $S$, Greiser M, Harks E, Blaauw $Y$, van Hunnik A, Verheule S et al. AVE0118, blocker of the transient outward current (I(to)) and ultrarapid delayed rectifier current (I(Kur)), fully restores atrial contractility after cardioversion of atrial fibrillation in the goat. Circulation 2006;114:1234-1242.

13. Ausma J, van der Velden HM, Lenders MH, van Ankeren EP, Jongsma HJ, Ramaekers FC et al. Reverse structural and gap-junctional remodeling after prolonged atrial fibrillation in the goat. Circulation 2003;107:2051-2058.

14. Ausma J, Wijffels M, Thone F, Wouters L, Allessie MA, Borgers M. Structural changes of atrial myocardium due to sustained atrial fibrillation in the goat. Circulation 1997;96:3157-3163.

15. Holm M, Johansson R, Brandt J, Luhrs C, Olsson SB. Epicardial right atrial free wall mapping in chronic atrial fibrillation. Documentation of repetitive activation with a focal spread--a hitherto unrecognised phenomenon in man. Eur Heart J 1997;18:290-310.

16. Kanagaratnam $\mathrm{P}$, Kojodjojo $\mathrm{P}$, Peters NS. Electrophysiological abnormalities occur prior to the development of clinical episodes of atrial fibrillation: observations from human epicardial mapping. Pacing Clin Electrophysiol 2008;31:443-453.

17. Gonzalez RC, Woods RE, Eddins SL. Digital Image Processing Using MATLAB. New Jersey: Prentice Hall, 2003.

18. Konings KT, Smeets JL, Penn OC, Wellens HJ, Allessie MA. Configuration of unipolar atrial electrograms during electrically induced atrial fibrillation in humans. Circulation 1997;95:1231-1241.

19. Eckstein J, Verheule S, de Groot N, Allessie MA, Schotten U. Mechanisms of perpetuation of atrial fibrillation in chronically dilated atria. Prog Biophys $\mathrm{Mol}$ Biol 2008;97:435-451.

20. de Groot NMS. Mapping and Ablation of Atrial TachyArrhythmias. Maastricht: Maastricht University. 2006.(Thesis)

21. Ausma J, Wijffels M, van Eys G, Koide M, Ramaekers F, Allessie MA et al. Dedifferentiation of atrial cardiomyocytes as a result of chronic atrial fibrillation. Am J Pathol 1997;151:985-997. 
22. Neuberger HR, Schotten U, Blaauw $Y$, Vollmann D, Eijsbouts S, van Hunnik A et al. Chronic atrial dilation, electrical remodeling, and atrial fibrillation in the goat. J Am Coll Cardiol 2006;47:644-653.

23. van der Velden HM, Ausma J, Rook MB, Hellemons AJ, van Veen TA, Allessie MA et al. Gap junctional remodeling in relation to stabilization of atrial fibrillation in the goat. Cardiovasc Res 2000;46:476-486.

24. Wang ZG, Fermini B, Nattel S. Repolarization differences between guinea pig atrial endocardium and epicardium: evidence for a role of Ito. Am J Physiol 1991;260:H1501-1506.

25. Van Wagoner DR, Pond AL, McCarthy PM, Trimmer JS, Nerbonne JM. Outward K+ current densities and Kv1.5 expression are reduced in chronic human atrial fibrillation. Circ Res 1997;80:772-781.

26. McFarlane Mood A, Graybill FA, Boes DC. Introduction to the Theory of Statistics. New York: McGraw-Hill Companies, 1974. 


\section{Chapter 4}

\section{Correlation between}

\section{Endo-epicardial Dissociation and}

\section{Transmural Conduction during Acute and}

\section{Persistent Atrial Fibrillation in the Goat}

Jens Eckstein ${ }^{\text {a b }}$, MD; Stef Zeemering ${ }^{a}$, MSc; Dominik Linz ${ }^{a}, M D ;$ Bart Maesen ${ }^{a}$, MD; Sander Verheule ${ }^{\mathrm{a}}$, PhD; Arne van Hunnik ${ }^{\mathrm{a}}$, BSc;

Harry Crijns ${ }^{a}, \mathrm{MD}, \mathrm{PhD}$, Maurits Allessie ${ }^{\mathrm{a}}, \mathrm{MD}, \mathrm{PhD}$; Ulrich Schotten ${ }^{\mathrm{a}}, \mathrm{MD}, \mathrm{PhD}$

a: Department of Physiology, University Maastricht, PO Box 616, 6200 MD Maastricht, The Netherlands,

b: Department of Medicine, Universityhospital Basel, Petersgraben 14, 4031 Basel, Switzerland 


\section{Abstract}

Background: Endo-epicardial dissociation (EED) of electrical activity has been hypothesized to contribute to the complexity of the substrate of atrial fibrillation (AF). The aim of this study was to investigate endo-epicardial differences in fibrillation wavefront characteristics in normal and remodelled atria, to study the correlation between endo-epicardial dissociation of electrical activity and the incidence of transmural conduction (breakthrough), and to test the plausibility of breakthrough to result from transmural conduction originating from the opposing layer of the atrial wall.

Methods and Results: We analyzed high-resolution simultaneous endo-epicardial mapping data recorded in left atrial free walls of 21 goats with acute AF, 3 weeks and 6 months AF (all $n=7$ ). Number, size, and width of fibrillation waves were analyzed. Waves were categorized according to their origin from outside (peripheral wave) or within (breakthrough) the mapping area.

The number of fibrillation waves in both the epicardial layer and the endocardial bundle network was comparable in all 3 groups and highest in the 6 months AF group. Incidence of breakthrough (\% of all fibrillation waves) was lowest $(2.2 \pm 1.0 \%)$ in acute $\mathrm{AF}$, higher $(12.7 \pm 6.0 \%)$ after 3 weeks and highest $(14.5 \pm 3.8 \%)$ after 6 months AF $(p<0.001)$. Breakthrough incidence was similar in the epicardial layer and the endocardial bundle network in all groups. Furthermore, breakthrough incidence positively correlated with the degree of endo-epicardial dissociation as well as with histological changes. $86 \%$ of all breakthroughs $(n=656)$ could be explained by transmural conduction considering all potential sources within a distance of $7 \mathrm{~mm}$ and assuming a minimal transmural conduction velocity of $20 \mathrm{~cm} / \mathrm{s}$ between the site of 
breakthrough and its potential source. Applying the same criteria for identification of simultaneous occurrence of breakthrough in the epicardial and endocardial layer suggests that focal discharges are less likely to underlie breakthrough during $\mathrm{AF}$ (13\% of all breakthroughs).

Conclusion: During the first months of $A F$, complexity of the substrate equally increases in the epicardial layer and the endocardial bundle network. Breakthrough incidence correlates with the degree of endo-epicardial dissociation of electrical activity. The far majority of breakthrough can be explained by transmural conduction. 


\section{Introduction}

Recently, electrical dissociation between muscle bundles and epicardial breakthrough were described as key elements of the AF substrate in both goats and patients with persistent $A F^{1-3}$ In these studies, the substrate of AF was described based on a quantitative assessment of the elementary units of the fibrillation process, the separate fibrillation waves. $^{3}$ It was demonstrated that due to progressive remodelling of the atria the width of the fibrillation waves declined while their number increased. Also, along with increasing complexity of the AF substrate, a higher rate of epicardial breakthrough was found in both goats and in patients with persistent AF. ${ }^{1,2}$ Konings et al. were the first to describe these events in mapping studies in patients with induced AF. ${ }^{4}$ They identified fibrillation waves that originated within the mapping area and presented with patterns of "radial spread of activation". Present hypotheses on mechanisms underlying these breakthrough events include transmural conduction from the opposing layer of the atrial wall (endo-epicardial or vice versa), local microreentry (rotors) or ectopic focal discharges as possible sources. $^{2,}$ 5-7 Dissociation of electrical activity between the endocardial bundle network and the epicardial layer (EED) during AF can be regarded as a prerequisite condition for transmural conduction of fibrillation waves because only in the presence of EED electrical activity in one layer can encounter excitable tissue in the opposing layer of the atrial wall. We recently demonstrated that in goats endo-epicardial activation time differences and differences in directions of conduction vectors increase during 6 months of $\mathrm{AF}^{8}$ However, data on characteristics of fibrillation waves propagating in the endocardial layer of the atrial wall and their spatio-temporal relation to epicardial waves are lacking so far. 
In this study, we investigated whether the properties of fibrillation waves differ between the endocardial bundle network and the epicardial layer at different stages of the remodelling process and whether, in analogy to longitudinal dissociation within the epicardial layer, ${ }^{3}$ EED is more pronounced in remodelled atria. Moreover, we investigated whether breakthrough occurs as often in the endocardium as in the epicardium and whether the breakthrough rate correlates with the degree of EED. Finally, we tested for the plausibility of breakthrough to be caused by transmural conduction, intramural re-entry, or ectopic focal discharges.

\section{Methods}

\section{Model}

In this study, the goat model of persistent AF was used. ${ }^{1,9,10}$ Three groups of goats were studied ( $n=7$ for each group). All goats including the sham-operated animals (acute $\mathrm{AF}=\mathrm{aAF}$ ) received a right atrial endocardial pacemaker lead (Medtronic Capsurefi $\left.{ }^{\circledR}\right)$. In the 3 weeks AF group (3WAF) and the 6 months AF group (6mAF), the endocardial pacemaker lead was connected to an implantable subcutaneous neurostimulator (Medtronic, Itrel $1^{\circledR}$ ). All animal procedures were conform to international standards on Research Animal Use and approved by the local ethical committee for animal experiments of Maastricht University. 


\section{Study Protocol}

Induction of AF

After a two week recovery period from neurostimulator implantation, AF was artificially induced and maintained by repetitive $50 \mathrm{~Hz}$ burst pacing at 3 times threshold every other second for 3 weeks in the $3 \mathrm{WAF}$ group. The $6 \mathrm{mAF}$ group was paced identically for the first 3 months, then the interval was changed to $1 \mathrm{~s} / \mathrm{min}$ for the remaining 3 months. No pacing was performed in the aAF group. Neurostimulators were switched off 1 hour before the experiments to check for spontaneous cardioversions.

\section{Open chest experiments}

Animals were anesthetized with sufentanyl $(6 \mathrm{mg} / \mathrm{kg} / \mathrm{h})$, midazolam $(0.8 \mathrm{mg} / \mathrm{kg} / \mathrm{h})$ and pancuronium $(0,3 \mathrm{mg} / \mathrm{kg} / \mathrm{h})$. No specific autonomic blockade was applied. After leftsided thoracotomy high-density endo-epicardial mapping of the left atrium was performed as described recently. ${ }^{8}$ Briefly, the lower part of a custom made clamplike mapping device (90 endocardial and 146 epicardial electrodes, electrodespacing $1.6 \mathrm{~mm}$ ) was inserted into the left atrial cavity of the beating heart through a small incision near the left atrial appendage. By carefully closing the clamp contact was made with the endocardial and epicardial layer of the atrial wall. The distance between the epicardial and the endocardial mapping array was narrowed until sharp unipolar electrogram were obtained at all electrode sites. A silver plate in the thoracic cavity served as indifferent electrode. Unipolar signals were digitized using a custom made 256 channel mapping amplifier (bandwidth $0.1-408 \mathrm{~Hz}$, sampling rate $1 \mathrm{KHz}$, resolution 16 bits) and stored for offline analysis. 


\section{Wave analysis}

AF-electrogram files of 4 seconds each were analyzed. Local activation times were identified as points of maximum negative $\mathrm{dV} / \mathrm{dt}$ in each unipolar electrogram. Identification of individual fibrillation waves followed an algorithm described earlier. ${ }^{1-3}$ Briefly, fibrillation waves were defined as areas surrounded by lines of block or fusion everywhere along their boundaries within the mapping array. Conduction block was assumed if the conduction time between neighbouring electrodes was more than $8 \mathrm{~ms}$ (or more than $12 \mathrm{~ms}$ in case of oblique electrodes) equivalent to a conduction velocity of $<20 \mathrm{~cm} / \mathrm{s}^{11,12}$ All resulting semi-automatic endo-epicardial wavemaps were reinspected and edited by two independent researchers.

Fibrillation waves were subsequently labelled as (1) peripheral waves if the site of earliest activation was at the border of the mapping area or (2) breakthrough if the site of earliest activation was located within the mapping area.

Apparent conduction velocity of fibrillation waves was determined by fitting planes through each activation time point and its neighbours (grid of $3 \times 3$ electrodes) including only activations that belonged to the same fibrillation wave. For each activation at each electrode, the local conduction vector was calculated from the orientation of the plane (local direction) and the reciprocal of the steepness of the plane (local conduction velocity). ${ }^{13}$ In case only two electrodes were allocated to a wave the conduction vector was defined by these two activations.

To quantify dissociation between waves in the epicardial and the endocardial layer, each electrode belonging to the same wave (according to the above mentioned algorithm) was analyzed for dissociated activity in time or direction in the closest nine electrodes in the opposing layer of the atrial wall. As a threshold for dissociation we 
used a calculated transmural conduction velocity of $<20 \mathrm{~cm} / \mathrm{s}$ for dissociation in time and a deviation of $>45^{\circ}$ between the corresponding conduction vectors for dissociation in direction of propagation. These threshold values are based on earlier work demonstrating a comparable distribution of activation time differences and differences in conduction direction for non-dissociated activity in aAF, 3WAF and $6 \mathrm{mAF}$ goats. ${ }^{8}$

Waves were then categorized as dissociated and non-dissociated. To obtain a robust distinction between these two populations of waves, waves were only categorized as dissociated if $80 \%$ or more of all activations were dissociated in time or direction in comparison to the corresponding electrodes in the opposing layer of the atrial wall and as non-dissociated if less then $20 \%$ of the activations were dissociated in time or direction. An example of a dissociated wave $(>80 \%$ of the surface dissociated) and a non-dissociated wave ( $<20 \%$ of the surface dissociated) is depicted in figure 1. Despite these strict criteria still $72 \%$ of all waves could be assigned to either the dissociated or the non-dissociated group. To study the effect of these thresholds on the quantification of EED we reanalyzed all data using less strict $(70 / 30)$ and more strict (90/10) dissociation threshold values. 


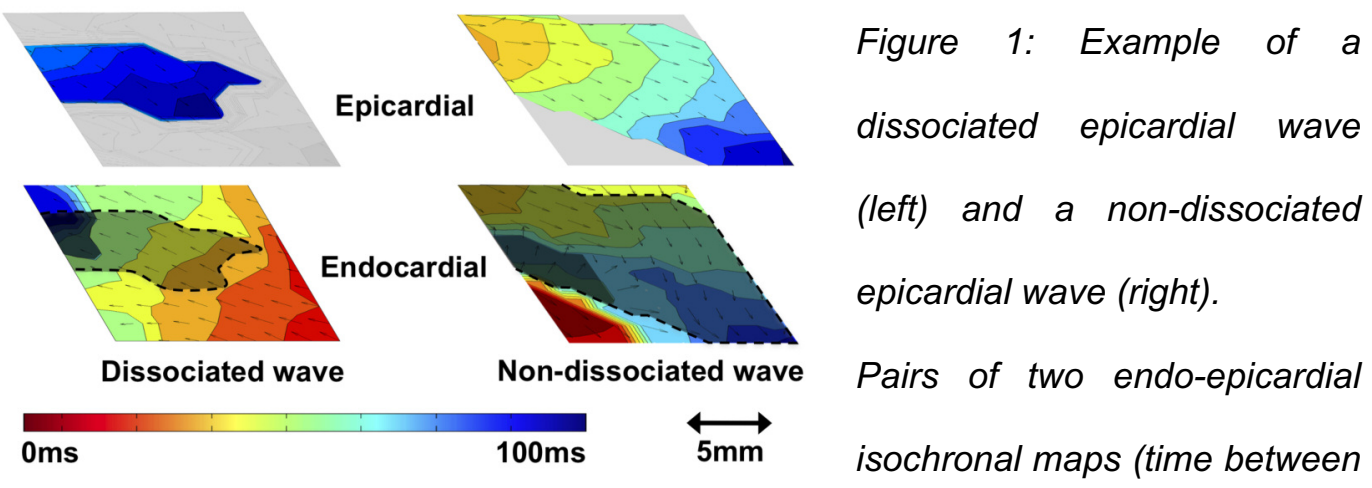

isochrones 5ms, red earliest, blue latest). Epicardial surfaces display a single wave. Outlines of the epicardial wave are projected on the endocardial map. Waves were compared for their activation times and directions of propagation to the opposing layer. If the overlap in time and direction was $<20 \%$ the wave was categorized as dissociated, if it was $>80 \%$ as non-dissociated.

The starting points of all breakthroughs were then tested for their closest contralateral activation time points. Plausibility of breakthroughs to result from transmural conduction from a contralateral source was calculated for a range of minimal transmural conduction velocities and the area within which an electrical activation was considered to be a potential source of the breakthrough. In the example depicted in figure 2 , an area of $5 \times 5$ electrodes $(8,0 \times 8,0 \mathrm{~mm})$ around the exactly opposing electrode was used for identification of preceding activations. If, for a given transmural conduction velocity, a preceding contralateral activation was detected that could have been a source of the breakthrough analysed, this breakthrough was categorized as "explainable by transmural conduction". 


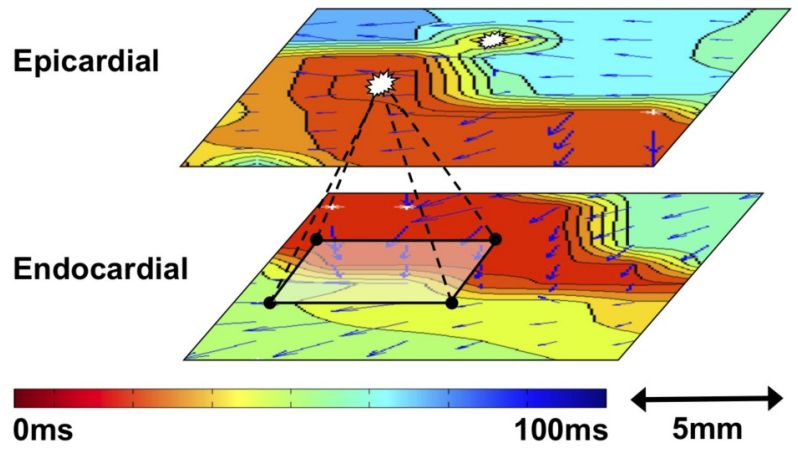

Figure 2: In the example, all sites of breakthrough (indicated by a white star) were evaluated for preceding activations of the closest 25 opposing electrodes (Grid $\left.5 \times 5=64 \mathrm{~mm}^{2}\right)$. Endocardial electrical activity is preceding the epicardial breakthrough by $6 \mathrm{~ms}$ (red area within the transparent box in the endocardial isochronal map, time between isochrones $5 m s$, red earliest, blue latest). The distance between endocardial and epicardial part of the mapping array was $2.7 \mathrm{~mm}$ reflecting a conduction velocity of $45 \mathrm{~cm} / \mathrm{s}$. This breakthrough would therefore be categorized as "explainable" by transmural conduction assuming a transmural conduction velocity of $45 \mathrm{~cm} / \mathrm{s}$ and a contralateral source area of $5 \times 5$ electrodes $\left(64 \mathrm{~mm}^{2}\right.$, space angle $\left.\approx 98^{\circ}\right)$.

To test the hypothesis of ectopic focal discharge as mechanism underlying breakthrough we analyzed the incidence of breakthrough that occurred simultaneously in the epicardial layer and the endocardial bundle network. The same range of contralateral source areas and transmural conduction velocities as described above was used to account for conduction from the site of ectopic focal discharge to the site of breakthrough.

Finally, we evaluated the possibility of intramural rotors (scroll waves) as sources for breakthrough events. For this purpose, the histogram of time intervals between each 
breakthrough and the latest preceding breakthrough within a $5 \times 5$ grid in the opposing layer of the atrial wall was plotted. In case of intramural scroll waves underlying breakthrough, an enhanced incidence of non-peripheral waves preceding the breakthrough by $\sim \mathrm{AFCL} / 2$ ( 60ms) would be expected.

\section{Histology}

After electrophysiological studies, hearts were excised, fixed in buffered $4 \%$ formaldehyde and imbedded in paraffin for histological evaluation. Tissue sections of $5 \mu \mathrm{m}$ were fixed at $56^{\circ} \mathrm{C}$ overnight, deparaffinized and rehydrated. To determine cardiomyocyte diameters and endomysial fibrosis, left atrial sections were stained with hematoxylin and eosin, and photographs were taken at 400x magnification. In each goat the diameter of the atrial myocytes was measured in 30-35 myocytes with the nucleus in plane of the section in each goat. Endomysial fibrosis was defined as connective tissue separating individual myocytes within bundles. The width of endomysial tissue septa was quantified as the distance between myocytes within bundles. ImageJ 1.37a (National Institute of Health, USA) was used for analysis.

\section{Statistical analysis}

All data are expressed as mean $\pm S D$. Significance of differences in means between $\mathrm{aAF}, 3 \mathrm{WAF}$, and $6 \mathrm{mAF}$ groups was calculated using a one way ANOVA with Newman-Keuls post-test and a paired Student t-test for significance of differences in means between the endocardial and the epicardial layer in the same specimen. $\mathrm{P}$ values $<0.05$ were considered significant. 


\section{Results}

\section{AF cycle length and conduction velocities}

Mean AFCL was $143 \pm 15 \mathrm{~ms}$ in the aAF group and shorter in the $3 \mathrm{wAF}$ group $(125 \pm 10 \mathrm{~ms}, \quad p<0.01)$ representing significant electrical remodelling. No further shortening of $A F C L$ was present in the $6 \mathrm{mAF}$ group $(122 \pm 5 \mathrm{~ms}$, n.s. compared to 3WAF). No significant difference was present between the endocardial and the epicardial layer in any of the three groups.

Mean conduction velocity of all conduction vectors was highest at baseline $(62 \pm 7 \mathrm{~cm} / \mathrm{s})$ slower after 3 weeks AF $(58 \pm 5 \mathrm{~cm} / \mathrm{s})$ and slowest after 6 months $A F$ $(51 \pm 4 \mathrm{~cm} / \mathrm{s}, p<0.01$ between all three groups, $p<0.01$ between aAF and $6 \mathrm{mAF}$, $p<0.05$ between $3 \mathrm{WAF}$ and $6 \mathrm{mAF}$ and $p>0.05$ between aAF and $3 \mathrm{WAF}$ ). No difference in overall conduction velocity was present between the endocardial and the epicardial layer (table 1). No spontaneous cardioversions occurred after switching of the neurostimulators $>1 \mathrm{~h}$ before the open chest experiments in any group.

\section{Wave characteristics analysis and endo-epicardial dissociation}

Total number of waves per AF-cycle was lowest in aAF, higher after 3 weeks AF and highest after 6months AF. Accordingly, the mean wave surface area showed a negative correlation. No significant differences in number or size of the waves were present between the endocardial and the epicardial layer (figure 3). 


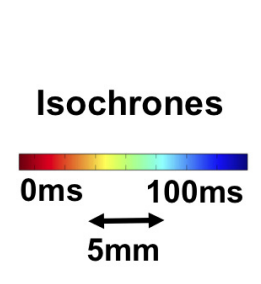

Wave size

(electrodes $=2.5 \mathrm{~mm}^{2}$ )

Number of Waves / AFCL

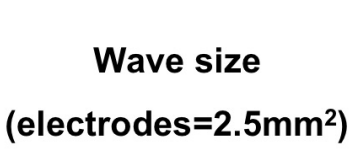

Acute
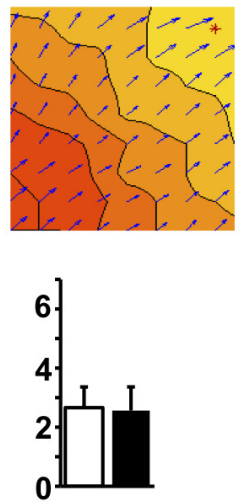

3 weeks
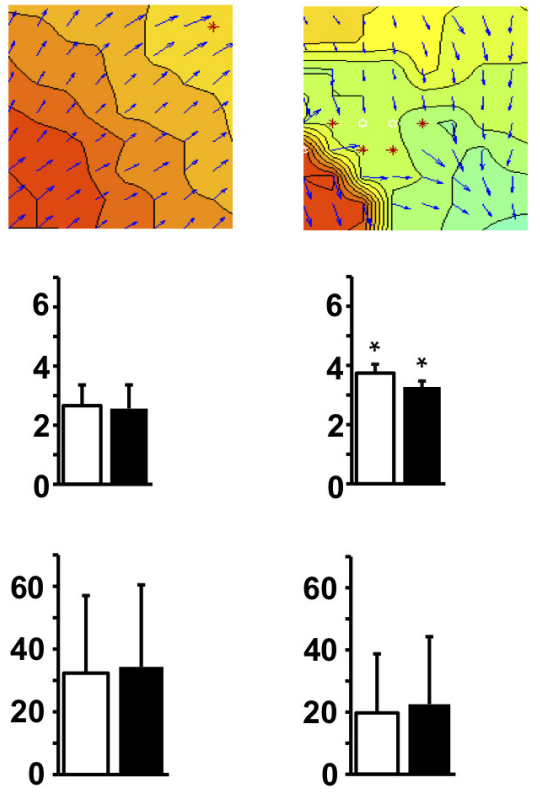

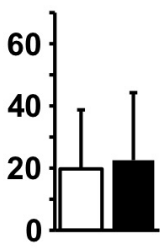

\section{6 months}
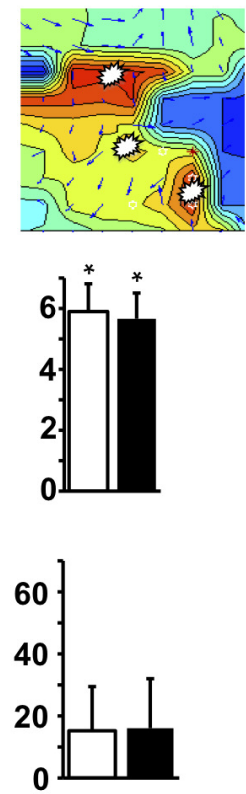

Epicardial

Figure 3: Atrial remodelling leading to inhomogeneous conduction and increasing incidence of breakthrough.

Isochronal maps in the upper row depict the increasing inhomogeneity of activation with AF duration (time between isochrones $5 \mathrm{~ms}$, earliest activations color coded in red, latest in blue, white stars representing sites of breakthrough). Graphs depict the increasing number of fibrillation waves/AFCL (middle row), and decreasing size of fibrillation waves with AF duration (lower row, x-axis depicting number of electrodes activated by the same wave), ${ }^{*}=$ significant v.s. aAF. 
While number and surface area of non-dissociated waves was highest in the aAF group and smallest in the 6mAF group, the number and surface area of dissociated waves showed a diametrically opposed development. Wave based analysis of EED demonstrated that $9 \pm 5 \%$ of all fibrillation waves were dissociated during acute $A F$, $17 \pm 5 \%$ after 3 weeks of $\mathrm{AF}$ and $29 \pm 7 \%$ after 6 months of $\mathrm{AF}$. This corresponded to $3 \pm 3 \%$ of the surface area analysed being activated by dissociated waves during acute $A F, 5 \pm 3 \%$ after 3 weeks of $A F$ and $14 \pm 6 \%$ after 6 months of $A F$ (table 1 ).

Testing the EED results for different dissociation thresholds (by changing the fraction of the wave surface required for the classification "dissociated") demonstrated, that the fraction of dissociated waves in the $6 \mathrm{mAF}$ group increases from $29 \%$ to $32 \%$ if only $70 \%$ (instead of $80 \%$ ) dissociated activations are required for dissociation and decreases to $24 \%$ if this threshold is set at $90 \%$. This analysis demonstrated that the increase in EED between groups is comparable independent of the threshold chosen and that the absolute values only moderately depend on the thresholds applied (figure 4). The fraction of waves not included in the analysis for meeting neither of the two thresholds was low in all three scenarios tested $(15 \%$ for $70 / 30,23 \%$ for $80 / 20$ and $35 \%$ for $90 / 10$ ) 
Figure 4: Impact of different

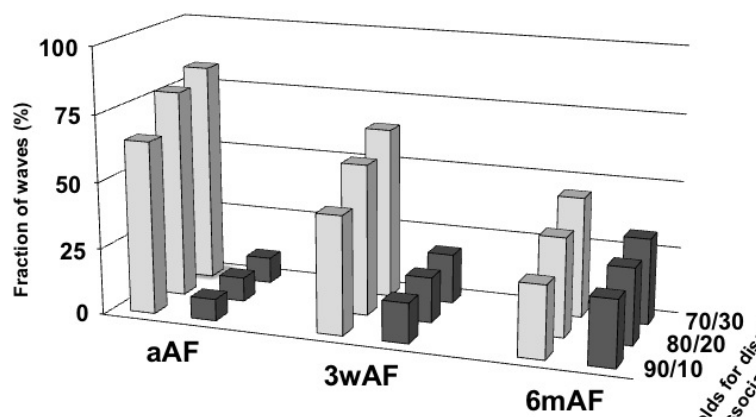

Non-dissociated

Dissociated thresholds for wave based analysis of EED. Also after applying less strict (70/30) and stricter (90/10) thresholds an increase in dissociated and a decrease in non-dissociated waves was

observed with increasing AF duration. Even for very strict thresholds $190 \%$ dissociated activity required to qualify for a dissociated wave and less than $10 \%$ dissociated activity required to qualify as non-dissociated wave) the majority of waves (65\%) could be included in either the dissociated or the non-dissociated group.

The incidence of breakthrough (as percentage of all waves) was lowest during acute AF $(2.2 \pm 1.0 \%)$, higher after 3 weeks of $\mathrm{AF}(12.7 \pm 6.7 \%)$ and highest after 6 months of $\mathrm{AF}(14.5 \pm 3.8 \%)$. There was a positive correlation between the incidence of breakthrough and the degree of EED (figure 5). Endocardial and epicardial incidences of breakthrough were not significantly different (table 1). 


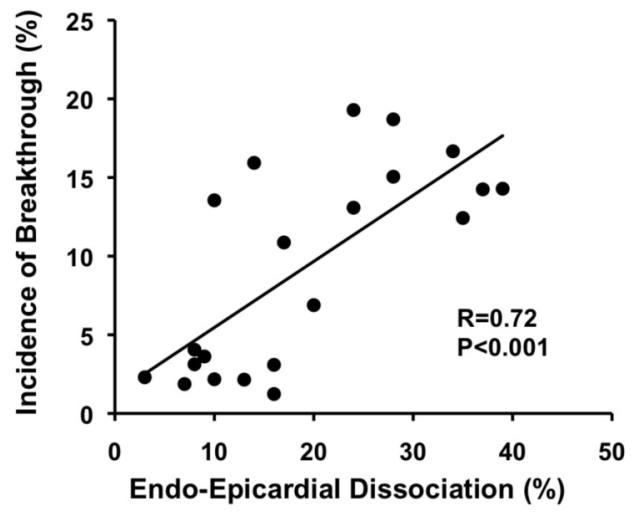

Figure 5: Correlation between EED and incidence of breakthrough.

$X$-axis depicting EED (\% dissociated waves of all waves), Y-axis depicting incidence of breakthrough (\% breakthrough of all waves). Each dot represents the result of one episode of $4 \mathrm{~s}$ in each of the 21 goats investigated.

\begin{tabular}{|c|c|c|c|c|c|c|}
\hline & \multicolumn{2}{|c|}{ Acute AF } & \multicolumn{2}{|c|}{3 weeks AF } & \multicolumn{2}{|c|}{6 months AF } \\
\hline Dissociated waves (\%) & \multicolumn{2}{|c|}{$9 \pm 5$} & \multicolumn{2}{|c|}{$17 \pm 5^{*}$} & \multicolumn{2}{|c|}{$29 \pm 7^{*} \dagger$} \\
\hline Non-dissociated Waves (\%) & \multicolumn{2}{|c|}{$78 \pm 10$} & \multicolumn{2}{|c|}{$57 \pm 11^{*}$} & \multicolumn{2}{|c|}{$37 \pm 9 * \dagger$} \\
\hline Surface Dissociated (\%) & \multicolumn{2}{|c|}{$3 \pm 3$} & \multicolumn{2}{|c|}{$5 \pm 3^{*}$} & \multicolumn{2}{|c|}{$14 \pm 6^{*} \dagger$} \\
\hline \multirow[t]{2}{*}{ Surface Non-dissociated (\%) } & \multicolumn{2}{|c|}{$88 \pm 11$} & \multicolumn{2}{|c|}{$70 \pm 13^{*}$} & \multicolumn{2}{|c|}{$41 \pm 13^{*} \dagger$} \\
\hline & endo & epi & endo & epi & endo & epi \\
\hline $\mathrm{AFCL}(\mathrm{ms})$ & $143 \pm 15$ & $142 \pm 15$ & $125 \pm 10^{*}$ & $124 \pm 11^{*}$ & $121 \pm 6^{*}$ & $123 \pm 5^{*}$ \\
\hline $\mathrm{CV}(\mathrm{cm} / \mathrm{s})$ & $62 \pm 8$ & $62 \pm 9$ & $58 \pm 6^{*}$ & $59 \pm 7^{*}$ & $51 \pm 5^{\star} \dagger$ & $51 \pm 3^{*} \dagger$ \\
\hline Breakthrough (\%) & $1.6 \pm 0.8$ & $2.8 \pm 1.9$ & $12.8 \pm 6.4^{*}$ & $12.6 \pm 7.0^{*}$ & $14.4 \pm 5.5^{*}$ & $14.7 \pm 6.1^{*}$ \\
\hline
\end{tabular}

Table 1: Time course of EED (calculated by fraction of waves and fraction of surface), endocardial and epicardial AFCL, conduction velocities and wave type during the atrial remodelling process. No significant differences were present for any of the parameters listed between endo- and epicardium at any time $\left(^{*}=\right.$ significant $v s$. Acute $A F, \dagger=$ significant vs. 3 weeks $A F)$. 


\section{Origin of breakthroughs}

In total we analyzed 5643 individual fibrillation waves and identified 656 breakthroughs (11.6\%). Three different combinations observed with breakthrough are depicted in figure 6 . While the majority of breakthroughs were preceded by contralateral activity, few were accompanied by a contralateral breakthrough or didn't show any simultaneous or preceding contralateral activity.

\section{Epicardial}
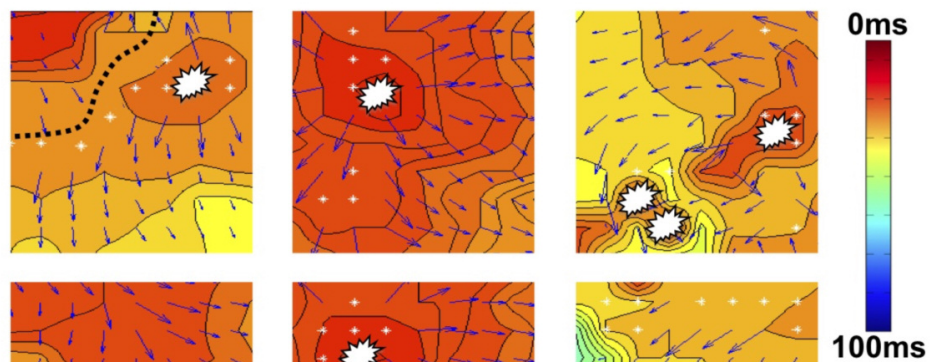

Endocardial

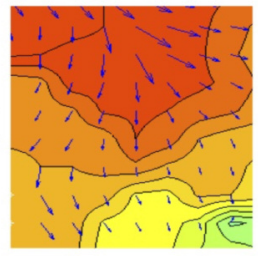

A

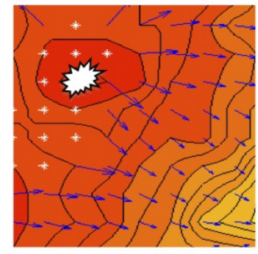

B

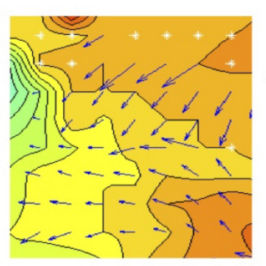

$100 \mathrm{~ms}$

C

Figure 6: Three isochronal maps from simultaneous endo-epicardial recordings.

All three show one or more epicardial "breakthrough" patterns (indicated by white stars), but differ in endocardial activation (time between isochrones $5 \mathrm{~ms}$, red earliest, blue latest).

Left panels: Breakthrough with preceding contralateral activation representing the majority of breakthrough events. A transmural fibrillation wave is slowed down in the epicardial layer (crowding of isochronal lines, upper left corner) and rapidly propagates endocardially from where it activates the epicardial layer and fuses with the delayed epicardial part of the initial wave (dotted line). 
Mid panels: Simultaneous endocardial and epicardial radial activation-pattern. Possible source for such an event could be either a "concealed" intramural bundle activating the endocardium and epicardium at the same site simultaneously, or focal ectopic discharge activating endo- and epicardium simultaneously.

Right panels: Epicardial pattern with radial spread of activation preceding the endocardial activation. Possible underlying mechanism could be the same as in the example depicted in the mid panels.

Plausibility to result from transmural conduction largely depended on (1) the distance between an activation regarded as potential source of a breakthrough and (2) the minimal conduction velocity between this source and the breakthrough. To visualize the impact of these two thresholds applied with our algorithm, we calculated the percentage of breakthroughs explainable by transmural conduction as a function of minimal transmural conduction velocity and distance between potential source and breakthrough (figure 7, left panel).

Based on the assumption of a minimal transmural conduction velocity $>20 \mathrm{~cm} / \mathrm{s}$ and a maximal distance of $7 \mathrm{~mm}, 86 \%$ of all breakthroughs could be explained by transmural activation originating from preceding contralateral fibrillation waves. The conduction velocity threshold chosen for the present analysis is intentionally not taking possible slower transverse conduction into account and thereby probably underestimates the fraction of breakthrough explainable by contralateral activity. ${ }^{14}$ The remaining $14 \%$ could not be explained by transmural activation based on our criteria and the present data. 
Performing the same analysis to test for focal ectopic discharge as possible underlying mechanism for breakthrough only $13 \%$ of all events could be explained (compared to $86 \%$ for transmural conduction). Again a calculation for a range of thresholds was performed to demonstrate the impact of the threshold values on the absolute results (figure 7 , right panel).

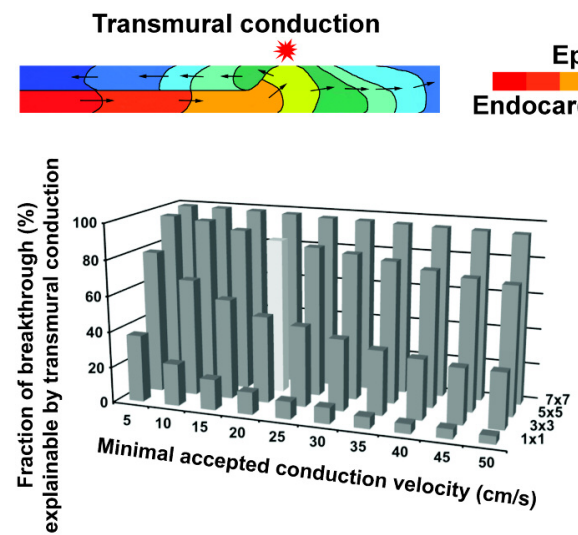

Epicardial layer

ndocardial bundle network
Ectopic focal discharge
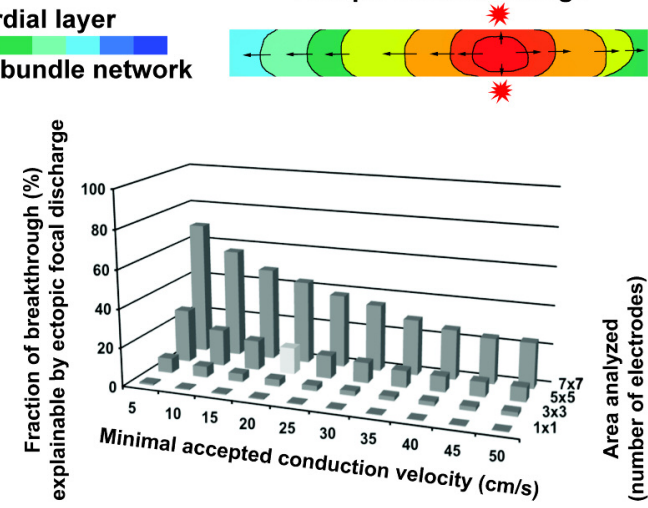

Figure 7: Impact of different thresholds for minimal accepted conduction velocity and grid sizes included in the analysis on the "explainability" of breakthroughs by transmural activation (left panel) and contralateral breakthrough/ectopy (right panel). $X$-axis $=$ minimal conduction velocity threshold $(\mathrm{cm} / \mathrm{s}), y$-axis $=\%$ of events explainable by transmural conduction, $z$-axis=size of area in the opposing layer of the atrial wall included in the analysis (number of electrodes included, interelectrode distance $1.6 \mathrm{~mm}$ ). Light grey columns depict the results based on the threshold used in the present analysis $(5 \times 5$ electrodes $=8 \times 8 \mathrm{~mm}, 20-100 \mathrm{~cm} / \mathrm{s})$. 
The histogram of the time intervals between the breakthrough and any preceding breakthrough showed a uniform decay with increasing intervals (figure 8). There was no increased incidence non-peripheral waves preceding contralateral breakthroughs by $\sim \mathrm{AFCL} / 2(\sim 60 \mathrm{~ms})$.

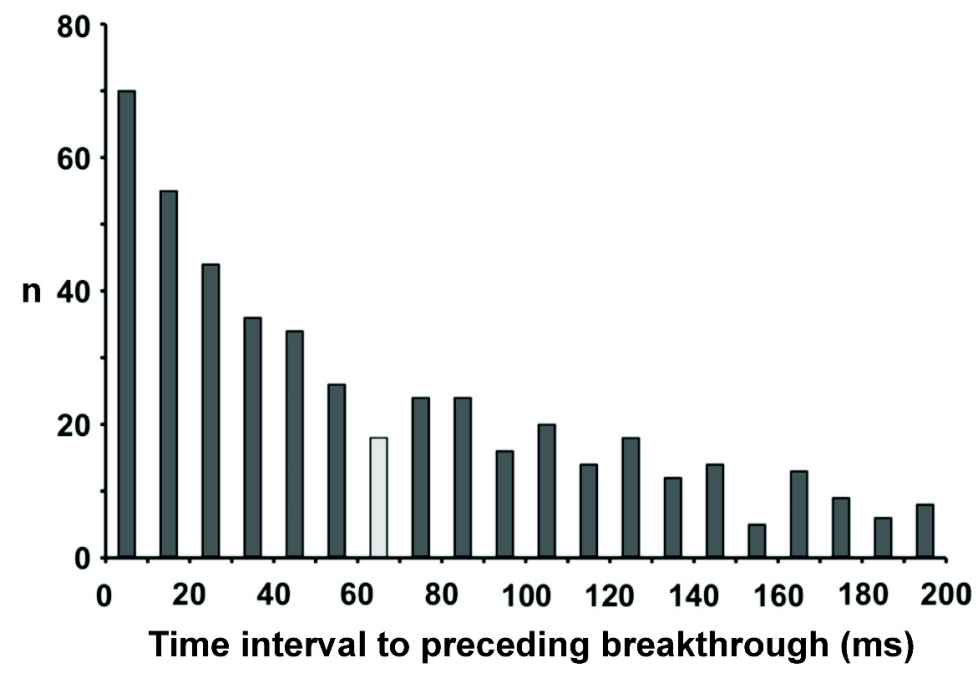

Figure 8: Activation time differences between starting points of breakthrough and contralateral preceding breakthrough. $Y$-axis depicts the number of events, $x$-axis depicts activation time differences between starting points of successive breakthrough events. No increased incidence of contralateral activations related to intramural scroll-waves at $\sim A F C L / 2(\sim 60 \mathrm{~ms}$, light grey bar) was present. 


\section{Histology}

The cell diameter as a parameter for myocyte hypertrophy, and the intermyocyte distances within bundles as a measure of enhanced extracellular matrix formation were significantly larger in the 6 months group compared to the sham group $(17.0 \pm 1.6 \mathrm{~mm}$ vs. $10.9 \pm 0.6 \mathrm{~mm}, p<0.05$ and $6.8 \pm 1.6 \mathrm{~mm}$ vs. $1.6 \pm 0.2 \mathrm{~mm}, p<0.01$ respectively, figure 9). No significant differences were present between the 3 weeks group and the Sham group. Both, the mean cell diameter as well as the intermyocyte distances correlated positively with the degree of endo-epicardial dissociation quantified as fraction of dissociated waves $(r=0.73$ and $r=0.55, p<0.05)$.
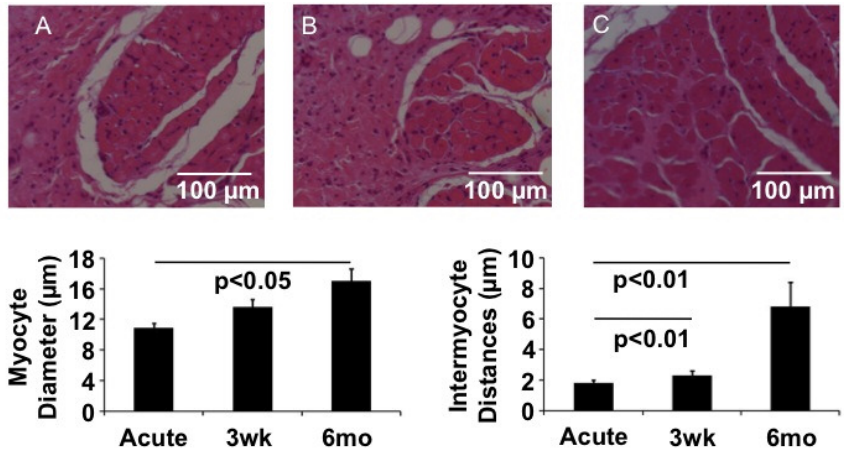

Figure 9: HE-stained LA sections of an aAF (A), a $3 w A F(B)$ and a $6 m A F(C)$ goat showing representative atrial bundles cut in the transverse direction. On the right, mean myocyte diameter (upper graph) and mean intermyocyte distances (lower graph) in aAF, 3WAF and 6mAF goats. The cell diameters as well as the intermyocyte distances within bundles as a measure of enhanced extracellular matrix formation were significantly larger in $6 m A F$ goats compared to aAF goats. 


\section{Discussion}

Previous experimental and clinical mapping studies described enhanced electrical dissociation and increased incidence of breakthrough with increasing complexity of the substrate of $\mathrm{AF}^{2}, 4,11$ These studies suggested transmural conduction of fibrillation waves rather than focal ectopic discharge as the leading mechanism of epicardial breakthrough. Endo-epicardial dissociation can be regarded as a prerequisite condition for transmural conduction. In this study, we provide a quantitative analysis of both, endocardial and epicardial fibrillation waves, as well as of their spatiotemporal relation to each other and to sites of breakthrough.

\section{Comparison of endocardial versus epicardial wave characteristics}

Besides the obvious anatomical differences between the epicardial layer and the endocardial bundle network, also the electrophysiological properties like refractory period and conduction velocities might differ between these two layers at different stages of the remodelling process. ${ }^{1,8}$ All these differences might contribute to endoepicardial differences in the conduction patterns during AF and to endo-epicardial dissociation of electrical activity. We therefore compared the endocardial with the epicardial wave propagation during acute and persisting AF. We found that the increasing complexity of the AF substrate, represented by more and narrower fibrillation waves, conduction slowing, and a higher incidence of breakthrough was present to an equal extent in the endo- and the epicardial layer of the atrial wall. Our study also demonstrated comparable incidences for epicardial and endocardial breakthroughs suggesting that transmural conduction does not have a preferential epi- to endocardial or endo- to epicardial direction in left atria of goats. 
These characteristics differ somewhat from indirect evidence in human AF where predominance of epicardial S-wave morphology of unipolar electrograms suggested preferential conduction from the epicardial layer to the endocardial bundle network. ${ }^{13}$

\section{Enhancement of EED of fibrillation waves and incidence of breakthrough during the development of the substrate of AF}

Our study confirms an earlier finding that with increasing complexity of the AF substrate endo-epicardial differences in activation time and direction increase. The presence of dyssynchrony in activation during AF was first demonstrated in AChinduced AF in perfused canine atria by Schuessler. ${ }^{15}$ We could recently confirm these findings and demonstrate more EED in persisting AF compared to acute AF based on endo-epicardial differences in local activation times and directions of conduction vectors. ${ }^{8}$ Moreover, we could demonstrate that the increase in EED with AF duration was independent of thresholds for conduction velocity and differences in direction. For the present study, we developed a wave-based algorithm for quantification of EED, with which we could not only quantify dissociation based on the relative number of dissociated fibrillation waves, but also demonstrate that the total area activated by dissociated fibrillation waves correlates with AF duration. In analogy with the previously defined dissociation index (length of block lines per area and time $)^{3}$ we defined an wave based index of EED as fraction of dissociated waves (as percentage of all waves and absolute surface area of dissociated waves). This index is sensitive for the total increase in number of fibrillation waves with $A F$ duration and the diametrically opposed changes in size of dissociated and nondissociated fibrillation waves. We could demonstrate that the relative number of 
dissociated waves, as well as the total area activated by dissociated waves correlates positively with AF duration. This result is of interest as EED is considered a conditio sine qua non for transmural conduction and the occurrence of breakthrough. In line with the hypothesis of transmural conduction as an underlying mechanism for breakthrough we could demonstrate a close correlation of EED with the incidence of breakthrough, which further supports the three-dimensional nature of the substrate in persistent AF.

Our study also shows that enhanced EED and breakthrough rate are related to electrical and progressive structural remodelling during 6 months of $A F$. In the present study, we compared goats with 3 weeks of AF with significant shortening of the AFCL due to electrical remodelling with goats after 6 months of AF in which we found no further shortening of the AFCL but myocyte hypertrophy and an increase in

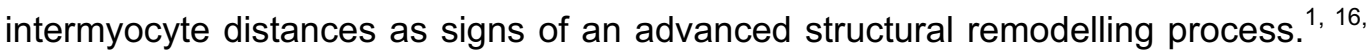
17 The fact that EED was much more pronounced after 6 months of AF compared to 3 weeks of AF without further shortening of the AF cycle length indicates, that electrical remodelling alone can not fully explain the large extent of EED after 6 months of AF and that structural alterations are likely contribute to EED as well. 


\section{Transmural conduction as mechanism underlying breakthrough}

The present study is the first to provide simultaneous endo-epicardial high-resolution in-vivo mapping data of breakthrough events. The analysis of this data shows that the plausibility of breakthrough to result from transmural conduction depends on assumptions of (1) the distance within which electrical activity is considered to potentially cause the breakthrough and (2) the minimal conduction velocity between this source and the breakthrough. For various reasons, for none of these parameters clear cut-off values can be deducted from our data or current electrophysiological knowledge. Transmural conduction might occur along the fibre direction or transverse to it meaning that conduction velocities between 5 and $120 \mathrm{~cm} / \mathrm{s}$ might occur. Slower velocities were reported for transverse conduction which would even increase the percentage of breakthroughs explainable by transmural activation. ${ }^{14}$ In structurally remodelled atria lower conduction velocities are possible. ${ }^{18,}{ }^{19}$ Furthermore, the exact distance a wave has to conduct from the endocardial bundles to the epicardial layer or vice versa remains unknown, as assessment of electrical activity within the atrial wall is not feasible with the techniques used in this study. Finally, it appears impossible to judge over what distance a fibrillation wave can propagate without being detected by the mapping electrodes used. Assuming a minimal conduction velocity of $20 \mathrm{~cm} / \mathrm{s}$ and a maximal distance between source and breakthrough of $7 \mathrm{~mm} 86 \%$ of all breakthroughs can be explained by transmural conduction. Any assumption of lower conduction velocity or longer distance between source and breakthrough would further increase this percentage.

In contrast, only $13 \%$ of all breakthrough events could be possibly related to a breakthrough in the contralateral layer as expected in case of ectopic focal 
discharges emphasizing that transmural conduction of fibrillation waves appears to be a much more likely explanation for the occurrence of breakthrough than ectopic activity. Testing for the plausibility of intramural rotors (scroll waves) as underlying mechanisms for breakthrough did not demonstrate a higher incidence of contralateral non-peripheral waves preceding the breakthrough with an interval of $\sim$ AFCL/2 ( 60ms). Thus, intramural rotors did not significantly contribute to breakthrough events in this study.

In the authors' view it is therefore reasonable to conclude that the far majority of breakthroughs can best be explained by transmural conduction. In a recent study of de Groot et al. in patients with AF a detailed analysis of epicardial mapping data for electrogram morphology (R-waves) and spatio-temporal distribution of breakthroughs led to the conclusion that the majority of breakthrough observed originates from transmural conduction rather than local ectopy. ${ }^{2}$ The non-repetitive occurrence and lack of prematurity further argued against focal ectopic discharges as the major source of breakthrough in this study.

Taken together these arguments are more in favour of transmural conduction as predominant source of breakthrough while cellular proarrhythmic mechanisms appear less likely. However, to which extent each of these two mechanisms contribute to the occurrence of breakthroughs cannot be determined exactly with the techniques currently available. 


\section{Role of three-dimensional conduction in AF stabilisation}

Figure 10 shows a hypothetical positive feedback loop of AF-stabilisation driven by EED and transmural conduction. According to this hypothesis, structural heart disease, as well as AF in itself, lead to electrical and structural remodelling resulting in disruption of side-to-side connections between muscle bundles. ${ }^{9,}{ }^{20}$ This results not only in conduction disturbances within the epicardial layer but also between the epicardial layer and the endocardial bundle network. More pronounced EED increases the likelihood for transmural conduction to occur and produces a 3dimensional substrate for AF. This increases the functional surface available for fibrillation waves to coexist and provides additional sites for re-entry. Both of these mechanisms are expected to increase the stability of AF.

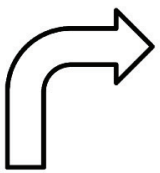

Number of Waves $\uparrow$<smiles>C1CCCC1</smiles>

3D Conduction (Breakthrough) $\uparrow$

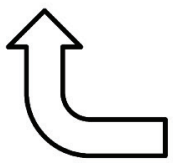

Endo-epicardial dissociation 1
Atrial Fibrillation

Conduction disturbances

Figure 10: Positive feedback loop highlighting the contribution of endo-epicardial dissociation and transmural conduction to the progressive nature of AF. 


\section{Potential limitations}

Testing plausibility of breakthroughs to result from transmural conduction by simultaneous endo-epicardial high-density mapping requires assumptions of 1 . the minimal transmural conduction velocity and 2 . the maximal distance between the site of earliest activation of a breakthrough and the potential source in the opposing layer of the atrial wall. Despite the fact that we were able to demonstrate simultaneous high density mapping data from the epicardial layer and the corresponding site of the endocardial bundle network and despite the fact the atrial wall was as thin as 2 to $3 \mathrm{~mm}$, direct proof of transmural conduction as the underlying mechanism of breakthrough is hampered by the uncertainty with which such assumptions can be made.

\section{Conclusion}

During the development of a substrate of AF, complexity of fibrillatory conduction increases in the epicardial layer and the endocardial bundle network to a comparable extent. Breakthroughs occur as often in the epicardial layer as in the endocardial bundle network. The degree of EED correlates with the breakthrough rate. The majority of breakthrough on the left atrial free wall of the goat is likely due to transmural conduction originating from the opposing layer of the atrial wall. This should be taken into account when targeting these sites with ablation therapy or evaluating the effect of potential antiarrhythmic drugs on triggered activity / automaticity. 


\section{References}

1. Verheule S, Tuyls E, van Hunnik A, Kuiper M, Schotten U, Allessie M. Fibrillatory conduction in the atrial free walls of goats in persistent and permanent atrial fibrillation. Circ Arrhythm Electrophysiol. 2010;3(6):590-599.

2. de Groot NM, Houben RP, Smeets JL, Boersma E, Schotten U, Schalij MJ, Crijns $\mathrm{H}$, Allessie MA. Electropathological substrate of longstanding persistent atrial fibrillation in patients with structural heart disease: epicardial breakthrough. Circulation. 2010;122(17):1674-1682.

3. Allessie MA, de Groot NM, Houben RP, Schotten U, Boersma E, Smeets JL, Crijns HJ. Electropathological substrate of long-standing persistent atrial fibrillation in patients with structural heart disease: longitudinal dissociation. Circ Arrhythm Electrophysiol. 2010;3(6):606-615.

4. Konings KT, Kirchhof CJ, Smeets JR, Wellens HJ, Penn OC, Allessie MA. High-density mapping of electrically induced atrial fibrillation in humans. Circulation. 1994;89(4):1665-1680.

5. Burashnikov A, Antzelevitch C. Late-phase 3 EAD. A unique mechanism contributing to initiation of atrial fibrillation. Pacing Clin Electrophysiol. 2006;29(3):290-295.

6. Haissaguerre $M$, Jais $P$, Shah DC, Takahashi A, Hocini M, Quiniou G, Garrigue S, Le Mouroux A, Le Metayer P, Clementy J. Spontaneous initiation of atrial fibrillation by ectopic beats originating in the pulmonary veins. $N$ Engl J Med. 1998;339(10):659-666.

7. Yamazaki M, Vaquero LM, Hou L, Campbell K, Zlochiver S, Klos M, Mironov $\mathrm{S}$, Berenfeld O, Honjo H, Kodama I, Jalife J, Kalifa J. Mechanisms of stretchinduced atrial fibrillation in the presence and the absence of adrenocholinergic stimulation: interplay between rotors and focal discharges. Heart Rhythm. 2009;6(7):1009-1017.

8. Eckstein J, Maesen B, Linz D, Zeemering S, van Hunnik A, Verheule S, Allessie M, Schotten U. Time course and mechanisms of endo-epicardial electrical dissociation during atrial fibrillation in the goat. Cardiovasc Res. 2011;89(4):816-824.

9. Wijffels MC, Kirchhof CJ, Dorland R, Allessie MA. Atrial fibrillation begets atrial fibrillation. A study in awake chronically instrumented goats. Circulation. 1995;92(7):1954-1968. 
10. Wijffels MC, Kirchhof CJ, Dorland R, Power J, Allessie MA. Electrical remodeling due to atrial fibrillation in chronically instrumented conscious goats: roles of neurohumoral changes, ischemia, atrial stretch, and high rate of electrical activation. Circulation. 1997;96(10):3710-3720.

11. Holm M, Johansson R, Brandt J, Luhrs C, Olsson SB. Epicardial right atrial free wall mapping in chronic atrial fibrillation. Documentation of repetitive activation with a focal spread--a hitherto unrecognised phenomenon in man. Eur Heart J. 1997;18(2):290-310.

12. Kanagaratnam $\mathrm{P}$, Kojodjojo $\mathrm{P}$, Peters NS. Electrophysiological abnormalities occur prior to the development of clinical episodes of atrial fibrillation: observations from human epicardial mapping. Pacing Clin Electrophysiol. 2008;31(4):443-453.

13. Houben RP, de Groot NM, Smeets JL, Becker AE, Lindemans FW, Allessie MA. S-wave predominance of epicardial electrograms during atrial fibrillation in humans: indirect evidence for a role of the thin subepicardial layer. Heart Rhythm. 2004;1(6):639-647.

14. Spach MS, Dolber PC. Relating extracellular potentials and their derivatives to anisotropic propagation at a microscopic level in human cardiac muscle. Evidence for electrical uncoupling of side-to-side fiber connections with increasing age. Circ Res. 1986;58(3):356-371.

15. Schuessler RB, Kawamoto T, Hand DE, Mitsuno M, Bromberg BI, Cox JL, Boineau JP. Simultaneous epicardial and endocardial activation sequence mapping in the isolated canine right atrium. Circulation. 1993;88(1):250-263.

16. Ausma J, Litjens N, Lenders MH, Duimel H, Mast F, Wouters L, Ramaekers F, Allessie M, Borgers M. Time course of atrial fibrillation-induced cellular structural remodeling in atria of the goat. $J \mathrm{Mol}$ Cell Cardiol. 2001;33(12):2083-2094.

17. Ausma J, Wijffels M, Thone F, Wouters L, Allessie M, Borgers M. Structural changes of atrial myocardium due to sustained atrial fibrillation in the goat. Circulation. 1997;96(9):3157-3163.

18. Neuberger HR, Schotten $U$, Blaauw $Y$, Vollmann D, Eijsbouts $S$, van Hunnik A, Allessie M. Chronic atrial dilation, electrical remodeling, and atrial fibrillation in the goat. J Am Coll Cardiol. 2006;47(3):644-653.

19. Spach MS, Heidlage JF, Barr RC, Dolber PC. Cell size and communication: role in structural and electrical development and remodeling of the heart. Heart Rhythm. 2004;1(4):500-515.

20. Spach MS, Heidlage JF, Dolber PC, Barr RC. Mechanism of origin of conduction disturbances in aging human atrial bundles: experimental and model study. Heart Rhythm. 2007;4(2):175-185. 


\section{Chapter 5}

\section{Effects of Flecainide on \\ Endo-Epicardial Dissociation and \\ Incidence of Breakthrough}

Jens Eckstein, MD, ${ }^{1}$ Bart Maesen, MD, ${ }^{1}$ Stef Zeemering, MSc, ${ }^{1}$ Dominik Linz, ${ }^{1}$ Arne van Hunnik, BSc, ${ }^{1}$ Sander Verheule, PhD, ${ }^{1}$ Harry J. Crijns, MD, $\mathrm{PhD},{ }^{2}$ Maurits Allessie, MD, PhD, ${ }^{1}$ Ulrich Schotten, MD, $\mathrm{PhD}^{1}$

1: Dept of Physiology, Maastricht University Medical Centre+, Maastricht, The Netherlands 2: Dept of Cardiology, Maastricht University Medical Centre+, Maastricht, The Netherlands

Presented at the annual meeting of the Heart Rhythm Society Heart Rhythm,2007. 4(Suppl 5): p. 175.

Presented at the annual meeting of the German society of Cardiology:

Clin Res Cardiol. March 2008;97(Suppl 1). 


\section{Abstract}

Background: The mechanism by which class IC drugs cardiovert atrial fibrillation $(\mathrm{AF})$ is not fully understood.

Methods: High-density simultaneous endo-epicardial mapping of AF was performed in vivo in the left atria of 3 groups of goats: acute $A F(a A F, n=7), 3$ weeks $A F(3 W A F)$ and 6 months $A F(6 m A F$, both $n=4)$. In the $3 w A F$ and $6 \mathrm{mAF}$ groups, data were acquired before and after administration of flecainide $(0.1 \mathrm{mg} / \mathrm{kg} / \mathrm{min}$, max. 90 minutes). Endo-epicardial dissociation (EED) was quantified based on endoepicardial activation time differences and differences in the direction of corresponding conduction vectors. Separate fibrillation waves were analyzed for their number, size and origin (peripheral vs. breakthrough). All breakthrough events were tested for the plausibility to originate from transmural conduction or from ectopic focal discharges.

Results: During acute AF, EED was $21 \pm 5 \%$. After 3 weeks of AF, EED was significantly higher than in aAF $(45 \pm 13 \%, p<0.01)$. Infusion of flecainide significantly reduced EED $(33 \pm 9 \%, \quad p<0.01$ compared to pre-flecainide) indicating partial resynchronization of electrical activity. After 6 months of AF, EED was significantly higher compared to the 3 WAF-group $(56 \pm 5 \%, p<0.01)$ and flecainide reduced EED to $37 \pm 18 \%$ ( $p<0.05$ compared to pre-flecainide). The incidence of breakthrough was higher in $3 \mathrm{WAF}$ and $6 \mathrm{mAF}$ group compared to the acute AF group. Flecainide reduced the breakthrough incidence to baseline level in both groups. Furthermore, it caused a larger reduction in the rate of breakthroughs occurring simultaneously at the endo- and epicardial layer of the atrial wall (presumably due to ectopic focal 
discharges, $-91 \%$ ) than in breakthroughs with either epicardial or endocardial occurrence (presumably due to transmural conduction, $-80 \%$ ). Wave size was not significantly affected by flecainide.

Conclusion: Dissociation of electrical activity between the epicardial layer and the endocardial bundle network is significantly higher after 3 weeks and 6 months of AF compared to acute AF. Partial resynchronization of electrical activity between the two layers, a reduction of transmural conduction and possibly an inhibition of ectopic focal discharges occurring during AF might contribute to the antiarrhythmic effect of flecainide during AF. 


\section{Introduction}

Sodium channel blocking agents have a long history as clinical compounds. First used as local anesthetics, they later were used as antiarrhythmic drugs to suppress ventricular premature beats that frequently occur after myocardial infarction. ${ }^{1}$ This was a standard treatment until the CAST trial (Cardiac Arrhythmia Suppression Trial) demonstrated enhanced mortality in the treatment group. ${ }^{2}$ While this trial ended the usage of flecainide for treatment of ventricular arrhythmias in patients with ischemic heart disease, it is still recommended and used successfully for cardioversion of atrial fibrillation (AF) (class $1 \mathrm{~A}$ recommendation) and maintenance of sinus rhythm. ${ }^{3}$ Despite their clear clinical efficacy, the exact mechanism leading to cardioversion by class $1 \mathrm{C}$ drugs still remains unclear.

While the effect of flecainide on atrial conduction velocity and AF cycle length (AFCL) has been thoroughly studied, only limited data is available on the effect of flecainide on fibrillatory conduction patterns, and more specifically on the threedimensional substrate of AF.

In patients with persistent $\mathrm{AF}$ as well as in the goat model of persistent $\mathrm{AF}$, the incidence of breakthrough events increases with AF duration. ${ }^{4-9}$ This mechanism presumably stabilizes the arrhythmia by initiating new wave fronts in the opposing layer of the atrial wall. Another mechanism most likely responsible for the increasing stability of AF over time is the increase of electrical dissociation between the epicardial layer and the endocardial bundle network (endo-epicardial dissociation $=$ EED), which increases the surface available for fibrillation wavelets to coexist and provides additional reentry points. ${ }^{8,}$ Chapter 4 In this study, we assessed the effect of flecainide on EED and the incidence of breakthroughs during AF. 


\section{Methods}

\section{Model}

For this study the goat model of persistent AF was used. ${ }^{8,10-12}$ Three groups of goats were studied: an acute $\mathrm{AF}$ group $(\mathrm{A} A \mathrm{~F}, \mathrm{n}=7)$ where $\mathrm{AF}$ was only induced during the open chest experiment, a three weeks AF group ( $3 \mathrm{WAF}, \mathrm{n}=4$ ) with 3 weeks of artificially maintained $A F$ and a 6 months $A F$ group $(6 \mathrm{mAF}, \mathrm{n}=4)$ with 6 months of artificially maintained AF. These goats form a subgroup of the animals included in chapters 3 and 4 .

\section{Study protocol}

\section{Induction of AF}

AF was induced and maintained as described earlier. ${ }^{8}$ Briefly, all animals of the $3 \mathrm{WAF}$ and $6 \mathrm{mAF}$ groups were implanted with a neurostimulator (Medtronic Itrel $1^{\circledR}$ ) and an endocardial right atrial electrode. In the 6mAF group, an additional pacemaker (Medtronic Kappa ${ }^{\circledR}$ ) and an additional endocardial lead (Medtronic Capsurefix ${ }^{\circledR}$ ) were implanted to obtain endocardial atrial electrograms during cardioversion experiments. After 2 weeks of recovery from surgery, neurostimulators were switched on to artificially maintain AF by repetitive burst pacing $(50 \mathrm{~Hz})$ every other second. In the $6 \mathrm{mAF}$ group, burst pacing was switched from $1 / \mathrm{s}$ to $1 /$ min after 14weeks. In the aAF group, AF was induced by burst pacing during the open chest experiment only. 


\section{Cardioversion experiments}

Pharmacological cardioversion experiments with flecainide $(0.1 \mathrm{mg} / \mathrm{kg} / \mathrm{min}$, i.v.) were performed in the 6mAF group to test for AF stability. Endpoints of the cardioversion experiments were cardioversion to sinus rhythm, ventricular arrhythmias, QRS prolongation of $>100 \%$, maximal duration of flecainide infusion of $90 \mathrm{~min}$ or visible discomfort of the animal. Neurostimulators were switched off at least one hour prior to the cardioversion experiments to test for incidence of spontaneous cardioversion.

\section{Open chest experiments}

Anesthesia during implantation and open chest experiments consisted of sufentanyl (6 mg/kg/h), midazolam (0.8mg/kg/h) and pancuronium (0.3mg/kg/h).

Synchronous endo-epicardial high-density mapping (246 electrodes, resolution $1.6 \mathrm{~mm}$ ) was performed in an open chest experiment using a custom made mapping tool as described earlier in more detail. ${ }^{8,13}$ Briefly, the endocardial part of a clamplike custom made mapping tool was inserted through a small incision close to the left atrial appendage into the left atrium. By closing the clamp carefully, contact with the epicardial and the endocardial atrial surface was achieved. Electrogram files were recorded during acute $A F$ in the aAF group. In the $3 \mathrm{WAF}$ and $6 \mathrm{mAF}$ groups electrograms were recorded before and during administration of flecainide $(0.1 \mathrm{mg} / \mathrm{kg} / \mathrm{min}$ for a maximum duration of $90 \mathrm{~min})$. The infusion was stopped earlier in case of cardioversion or repetitive ventricular arrhythmias. These arrhythmias were in most cases regular and non-sustained, but hemodynamically relevant. The last file of AF was used for analysis. 


\section{Data analysis}

Fibrillation electrogram files of four seconds were analyzed. Marking of local activations and demarcation of individual fibrillation waves was performed in a semiautomatic manner using custom made software as reported earlier in more detail. ${ }^{7,8}$ Briefly, fibrillation waves were defined as areas surrounded by lines of block or fusion everywhere along their boundaries within the mapping array. Conduction block was assumed if the conduction time between neighbouring electrodes was more than $8 \mathrm{~ms}$ (or more than $12 \mathrm{~ms}$ in case of oblique electrodes) equivalent to a conduction velocity of $<20 \mathrm{~cm} / \mathrm{s}^{14,15}$ All resulting semi-automatic endo-epicardial wavemaps were reinspected and edited by two independent researchers.

Endo-epicardial dissociation was quantified based on endo-epicardial activation time differences and endo-epicardial differences in direction of conduction of corresponding epi- and endocardial conduction vectors as described earlier in more detail. $^{8}$ Briefly, histograms of all endo-epicardial activation time differences and differences in direction of conduction vectors of corresponding endo-epicardial electrodes were analyzed. From the resulting histograms two distinct populations (i.e. dissociated and non-dissociated activity) could be identified by least square regression analysis of a two-component Gaussian function (for activation time differences), or a Gaussian/uniform mixture distribution (for differences in direction) to the histograms. The percentage of the area under the curve formed by the dissociated component was referred to as "dissociation index".

Local conduction velocity was determined by fitting planes through each activation time point and its neighbours (max. square of $5 \times 5$ electrodes). Only activation times belonging to the same wave were included in this analysis to avoid erroneous 
quantification of conduction by contribution of activation times beyond lines of conduction block. The resulting plane then indicated local direction (orientation of the plane) and velocity (reciprocal of the steepness of the plane) for each local activation point.

All individual fibrillation waves were analyzed for their size and classified as:

1. "peripheral waves" if the earliest activation time point of a wave was at the border of the mapping area, and

2. "breakthrough" if the earliest activation time point was located within the mapping area. ${ }^{\text {Chapter } 4}$

All breakthroughs were tested for the plausibility to derive from contralateral electrical activity or ectopic focal discharge. The general concept to study the explainability of breakthroughs by transmural conduction from preceding contralateral waves as possible sources by the use of synchronous endo-epicardial mapping data was described earlier by our group. As described in chapter 4 the percentage of breakthroughs explainable by transmural conduction largely depends on assumptions of the distance between the contralateral source and the site of breakthrough as well as on the minimal transmural conduction velocity.

The same holds true when a contralateral breakthrough is considered as the potential source of a breakthrough and "simultaneous" endo-epicardial occurrence is used as an argument for ectopic focal discharges as underlying mechanism. Therefore, both for testing plausibility of breakthroughs to arise from transmural conduction or ectopic focal discharges a variety of distances between source and site of breakthrough and minimal transmural conduction velocities was used as explained in chapter 4 . 
All animal procedures were performed in accordance with national and international guidelines and approved by the local ethics committee of Maastricht University.

\section{Statistical analysis}

All data are expressed as mean $\pm S D$. Significance of differences in means between aAF, 3WAF, and 6mAF groups were calculated using a one-way ANOVA with Newman-Keuls post-test and a unpaired Student's t-test for significance of differences in means between the endocardial and the epicardial layer in the same specimen and a paired Student's t-test for significance of differences before and after flecainide. P-values of $<0.05$ were considered significant. 


\section{Results}

Effect of flecainide on AF stability, AF cycle length, and conduction velocity Cardioversion experiments in awake goats

The average weight of all goats was $62.6 \pm 11.3 \mathrm{~kg}$. There was no significant difference between the groups $(p=0.49)$.

All goats of the $6 \mathrm{mAF}$ group were in persistent AF ( $>1 \mathrm{~h})$ after 2 weeks of artificially maintained AF. After 2 weeks of AF, 3/4 animals cardioverted to sinus rhythm during flecainide infusion. After 6 and 10 weeks, only 1 out of 4 goats cardioverted to sinus rhythm and after 14 weeks of AF no cardioversions were observed. In none of the animals, cardioversion occurred during the $>1 \mathrm{~h}$ time interval between switching of the stimulator and begin of the flecainide infusion.

\section{Open chest experiments}

Average AFCL during open chest experiments was $142 \pm 13 \mathrm{~ms}$ in the aAF group and $122 \pm 10 \mathrm{~ms}$ in the $3 \mathrm{WAF}$ group indicating significant electrical remodeling after 3 weeks of persistent AF $(p<0.05)$. No further difference was noted in the $6 \mathrm{mAF}$ group $(123 \pm 7 \mathrm{~ms}, \mathrm{p}=$ n.s. compared to $3 \mathrm{WAF}$ group). Flecainide prolonged $\mathrm{AFCL}$ during open chest experiments to $178 \pm 9 \mathrm{~ms}$ in the $3 \mathrm{WAF}$ group and $196 \pm 28 \mathrm{~ms}$ in the $6 \mathrm{mAF}$ group (figure 1). Two animals in the $3 W A F$ group cardioverted during flecainide administration. In these two goats AFCL before cardioversion was $164 \mathrm{~ms}$ and $173 \mathrm{~ms}$. None of the animals of the $6 \mathrm{mAF}$ group cardioverted during flecainide infusion. Flecainide infusion was stopped before the time limit of 90 minutes due to ventricular arrhythmias (all non sustained, regular, hemodynamically relevant ventricular tachycardias, after $75 \pm 12$ min infusion-time) in all open chest experiments 
except the 2 successful cardioversions in the 3wAF group. Conduction velocity during AF showed a trend to decrease with AF duration between the three groups and significantly decreased after flecainide administration (figure 2).

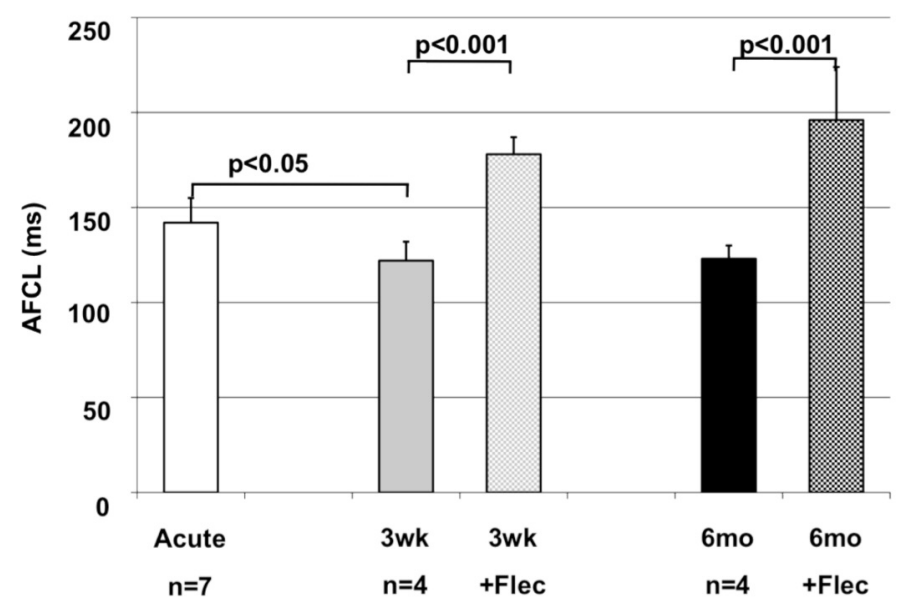

Figure 1: Mean AFCL during open chest experiments before and during flecainide administration. AFCL was significantly shorter after 3 weeks of AF compared to acute AF. No significant difference was present between 3 weeks $A F$ and 6 months $A F$. Flecainide prolonged AFCL to values above the AFCL in the acute AF group.

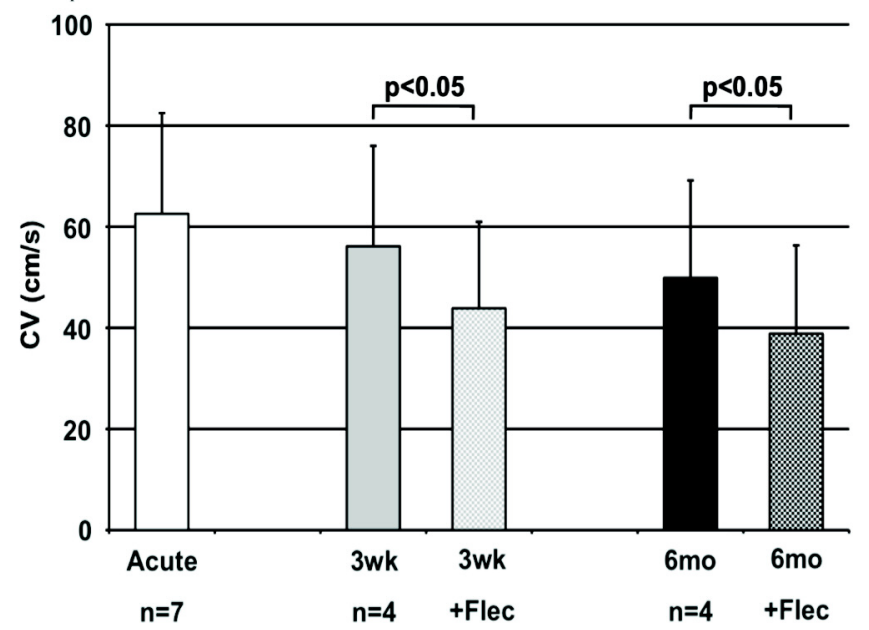

Figure 2: Mean conduction velocity during open chest experiments before and after administration of flecainide. Conduction during $A F$ showed a trend to decrease with $A F$ duration and was significantly reduced after

administration of flecainide. 


\section{Effect of flecainide on number and size of fibrillation waves}

An example of epicardial electrogram tracings and isochronal maps before and after flecainide administration is given in figure 3 . While prolongation of AFCL and the reduced conduction velocity due to flecainide administration were obvious, no changes in wave characteristics concerning wave size or width were present.

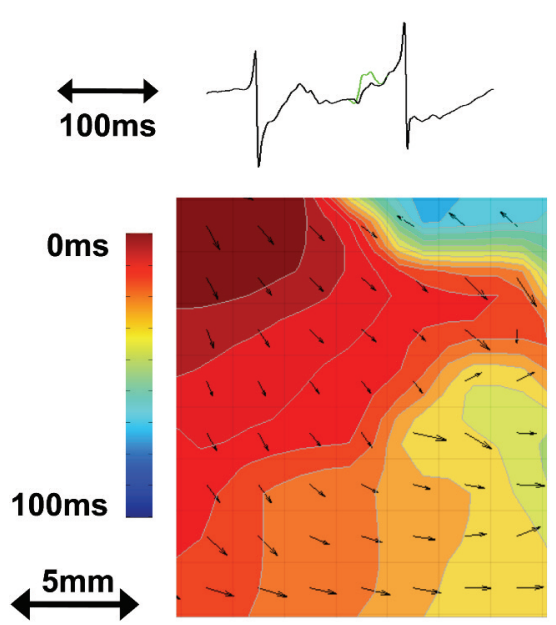

Acute AF
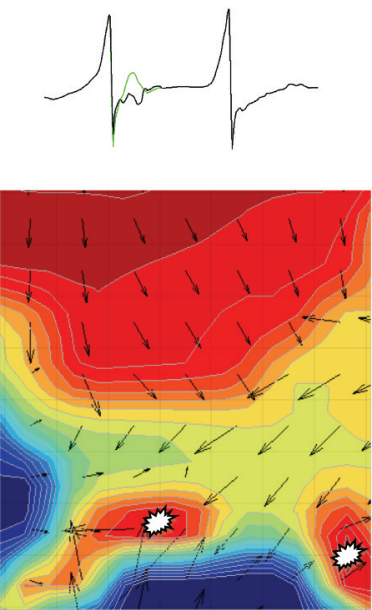

6 months AF
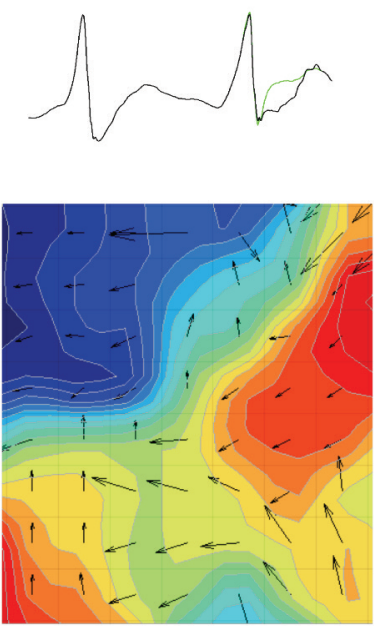

6 months AF

+ Flecainide

Figure 3:

Upper panels: Electrograms show a shorter AFCL $(122 \pm 10 m s, n=4)$ after 6 months $A F$ compared to acute $A F(142 \pm 13 m s, n=7)$. In the $6 m A F$ group, flecainide infusion produced a prolongation of the AFCL to values higher than found in the aAF group $(196 \pm 28 m s, n=4)$. The green lines represent tracings before subtraction of the ventricular farfield signal.

Lower panels: Isochronal maps demonstrate fast homogeneous conduction of fibrillation waves in the aAF group and an increasing complexity with breakthroughs (white stars), narrow waves and increased incidence of conduction block in the 
example of a $6 m A F$ goat. After administration of flecainide breakthrough incidence is significantly reduced and conduction velocity decreases.

Analysis of the number of waves simultaneously present in the mapping area showed 2.0 \pm 0.4 waves during acute $A F, 2.6 \pm 0.4$ waves after 3 weeks $A F$, and $2.9 \pm 0.7$ waves after 6 months AF. Flecainide reduced the number of waves simultaneously present to control values $(2.0 \pm 0.1$ waves in the $3 \mathrm{WAF}$ group and $1.9 \pm 0.2$ waves in the $6 \mathrm{mAF}$ group, figure 4$)$.

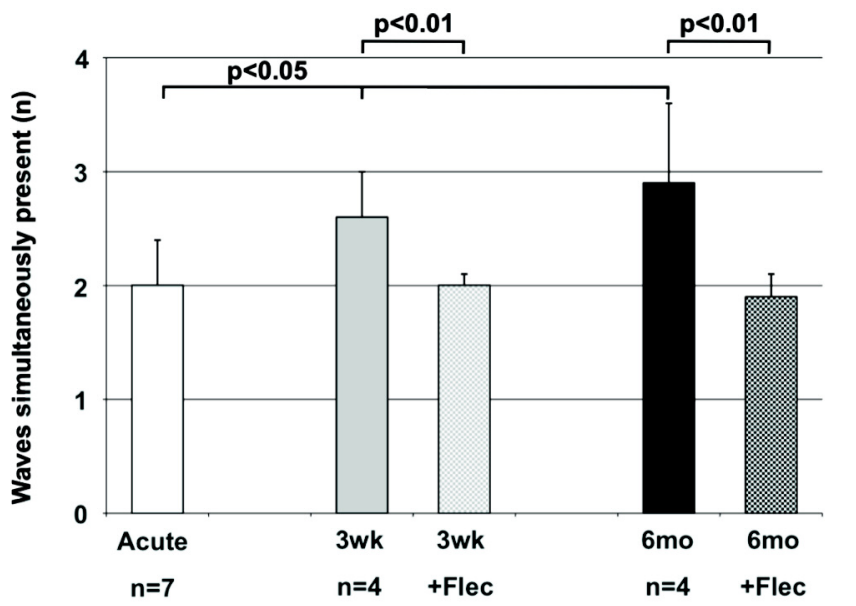

Figure 4: Number of waves simultaneously present within the mapping area in the aAF group (white bar), and before and after flecainide administration in the $3 W A F$ (grey bar, grey/white bar) and $6 m A F$ group (black bar, black/white bar). Y-axis depicts number of waves simultaneously present.

Because of the significant prolongation of AFCL by flecainide, we repeated the analysis above and corrected for AFCL. We also normalized for the surface area of the mapping electrode. The number of waves $/ \mathrm{cm}^{2}$ occurring during one AF cycle (1 $\mathrm{x}$ AFCL) was then $0.9 \pm 0.04$ during acute $A F, 1.7 \pm 0.08$ after 3 weeks $A F$ and $2.0 \pm 0.1$ after 6 months AF. Administration of flecainide resulted in a prolongation of the AFCL 
and a comparable decrease in the number of waves present per time. As a consequence, the total number of waves $/ \mathrm{cm}^{2}$ during one $\mathrm{AF}$ cycle $(1 \times \mathrm{AFCL})$ was not significantly altered $(1.8 \pm 0.08$ in the $3 \mathrm{WAF}$ group and $2.2 \pm 0.16$ in the $6 \mathrm{mAF}$ group, figure 5).

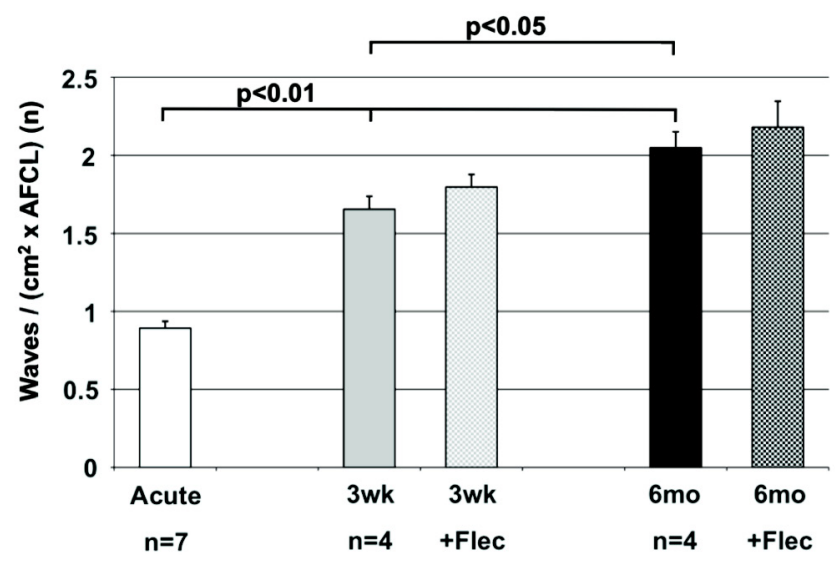

Figure 5: Number of waves / $\mathrm{cm}^{2}$ occurring during one $A F$ cycle in the aAF group (white bar), and before and during flecainide administration in the 3WAF (grey bar, grey/white bar) and $6 \mathrm{mAF}$ group (black bar, black/white bar). Y-axis depicts number of waves $/ \mathrm{cm}^{2}$ occurring during $1 \mathrm{AFCl}$.

The size of waves was largest in acute AF $\left(94.4 \pm 19.2 \mathrm{~mm}^{2}\right)$, smaller in the $3 \mathrm{WAF}$ group $\left(49.4 \pm 11.2 \mathrm{~mm}^{2}\right)$ and smallest in the $6 \mathrm{mAF}$ group $\left(39.4 \pm 5.8 \mathrm{~mm}^{2}, p<0.001\right.$ compared to aAF). Administration of flecainide did not influence wave size significantly in the $3 \mathrm{WAF}$ or the $6 \mathrm{mAF}$ group $\left(50.4 \pm 21.5 \mathrm{~mm}^{2}\right.$ and $33.6 \pm 9.4 \mathrm{~mm}^{2}$, $p=$ n.s., figure 6). Similar changes were found for the wave width, which was smaller after 3 weeks and 6 months of AF (aAF: $6.8 \pm 3.8 \mathrm{~mm}, 3 \mathrm{WAF}: 4.9 \pm 3.8 \mathrm{~mm}, 6 \mathrm{mAF}$ : $4.4 \pm 4.4 \mathrm{~mm}, \mathrm{p}=\mathrm{n} . \mathrm{s}$.). Flecainide did not affect wave width in $3 \mathrm{wAF}$ and $6 \mathrm{mAF}$ (3wAF: 4.8 $\pm 3.2 \mathrm{~mm}, 6 \mathrm{mAF}: 3.8 \pm 2.7 \mathrm{~mm}, \mathrm{p}=$ n.s. compared to wave width before flecainide). 


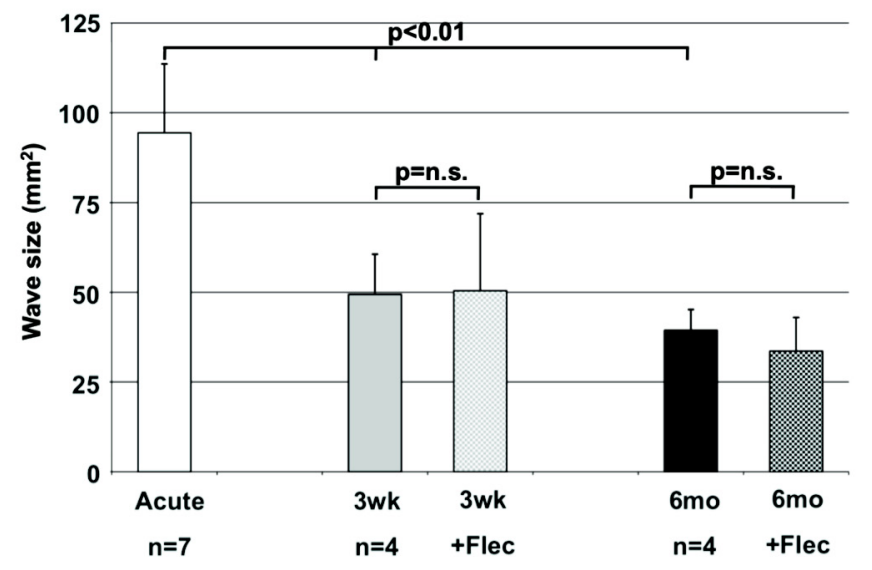

Figure 6: Effect of flecainide on wave size.

Y-axis: Average wave size in $\mathrm{mm}^{2}$ in the aAF group, and before and after flecainide administration in the $3 w A F$ and $6 m A F$ group. While the size of waves decreased with AF duration, flecainide did not affect wave size significantly. Maximum wave size that could theoretically be documented with the mapping device used is $230.4 \mathrm{~mm}^{2}$.

Effect of flecainide on endo-epicardial dissociation and incidence of breakthrough

While no significant EED was found during sinus rhythm, significant dissociation was present during acute AF (23 $\pm 9 \%)$. After 3 weeks of artificially maintained AF, EED was significantly higher $(45 \pm 13 \%, p=0.01$ compared to acute $A F)$. Infusion of flecainide significantly reduced EED $(33 \pm 9 \%, p=0.01$ vs. pre-flecainide) indicating partial resynchronization of electrical activity between the epicardial layer and the endocardial bundle network. EED in the 6mAF group was significantly higher compared to the $3 \mathrm{WAF}$ group $(56 \pm 5 \%, \mathrm{p}=0.01)$. Infusion of flecainide resulted in an EED reduction to $37 \pm 18 \%$. There was no significant difference in EED between acute $\mathrm{AF}$, and the $3 \mathrm{WAF}$ group and $6 \mathrm{mAF}$ group after administration of flecainide $(p=0.17$ compared to control, figure 7$)$. 


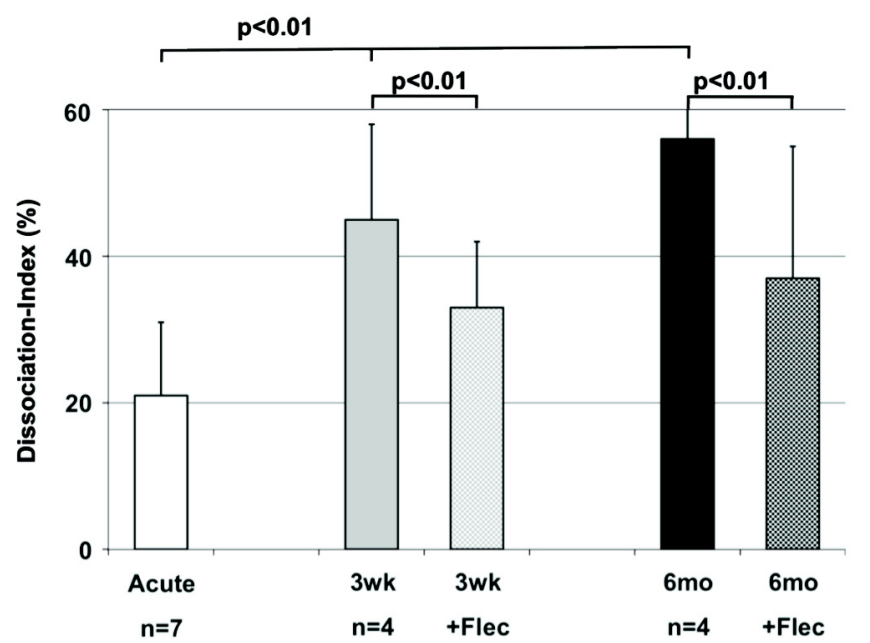

Figure 7: Effect of flecainide on EED.

Y-axis: Dissociation index calculated from activation time differences.

Dissociation was lowest in the control group (white bar), higher in the 3WAF group (grey bar) and highest in the 6mAF group (black bar). Administration of flecainide reduced dissociation in the 3WAF group (grey/white bar) and 6mAF group (black/white bar) to values not significantly different from the aAF group.

In the aAF group $2.3 \pm 1.0 \%$ of all fibrillation waves were breakthrough events. After 3 weeks of AF breakthrough incidence increased to $14.8 \pm 8.8 \%$. Administration of flecainide resulted in a decrease of breakthrough incidence to $4.7 \pm 3.2 \%$. After 6 months of persisting AF, $15.1 \pm 1.6 \%$ of all fibrillation waves were breakthrough events ( $p=$ n.s. vs. $3 w A F)$. Administration of flecainide reduced the incidence to $4.8 \pm 2.3 \%$ in this group (figure 8 ). 
Figure 8: Incidence of

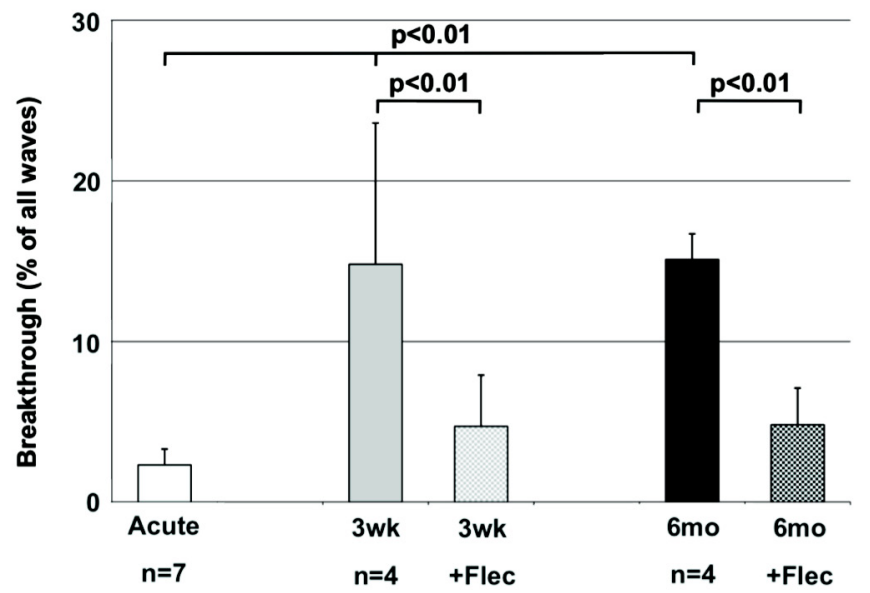
breakthrough events in the aAF group (white bar), and before and during flecainide administration in the $3 W A F$ (grey bar, grey/white bar) and 6mAF groups (black bar, black/white bar). Y-axis depicts \% of breakthrough of

all waves. No significant differences were present for breakthrough incidence in the control group compared to $3 W A F+f l e c a i n i d e$ and $6 m A F+f l e c a i n i d e$.

Analysis of all breakthrough events for the plausibility to originate from transmural conduction or focal ectopy showed that before flecainide administration $88 \%$ of all breakthroughs could be explained by transmural conduction and $19 \%$ could be explained by ectopic focal discharge (assuming a maximal distance from breakthrough to source of $7 \mathrm{~mm}$ and a minimal transmural conduction velocity of $20 \mathrm{~cm} / \mathrm{s}$, light grey bar in upper panels of figure 9). Flecainide not only reduced the total incidence of breakthroughs in all three groups but specifically reduced the percentage of breakthroughs that putatively originated from ectopic focal discharge to $8 \%$ (v.s 19\% before flecainide) while the percentage of breakthroughs that putatively originated from transmural conduction was not significantly altered $(88 \%$ 
before vs. $80 \%$ after flecainide, light grey bars in lower panels of figure 9). Importantly, preferential reduction of simultaneous endo-epicardial breakthroughs (indicating ectopic focal discharges) by flecainide was largely independent from the distance between putative source and site of breakthrough (absolute distances between the two electrodes at the starting point of breakthroughs) and the minimal transmural conduction velocity (figure 9). For a grid size of $5 \times 5$ contralateral electrodes $(8 \times 8 \mathrm{~mm})$ the strongest relative reduction $(86 \%)$ was found at $30 \mathrm{~cm} / \mathrm{s}$, while the weakest $(73 \%)$ was present at $5 \mathrm{~cm} / \mathrm{s}$. This reduction was still comparable to the reduction at $20 \mathrm{~cm} / \mathrm{s}(78 \%)$. 

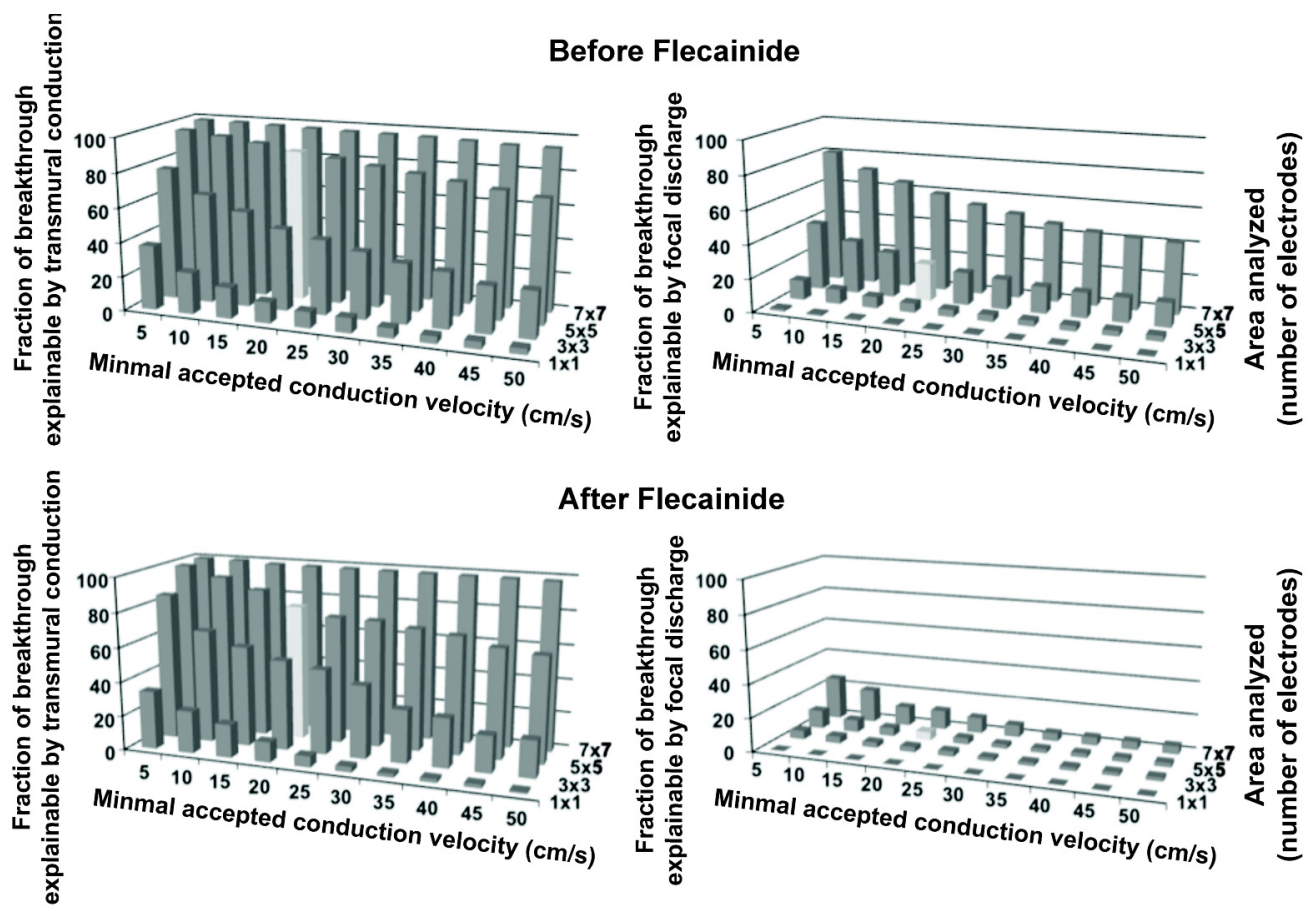

After Flecainide

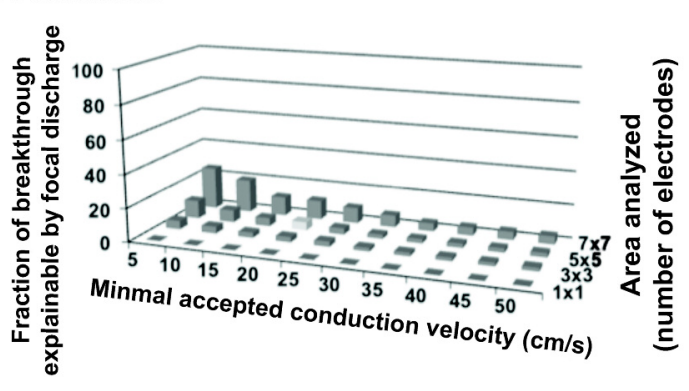

Figure 9: Effect of flecainide on the likelihood of breakthroughs to originate from contralateral waves versus ectopic focal discharge as a function of different thresholds for the lowest accepted conduction velocity (x-axis) and the grid size of electrodes on the contralateral side included in the analysis (z-axis). Y-axis depicts the percentage of breakthrough explainable based on the thresholds chosen. Results based on the thresholds applied in the present analysis are depicted in light grey bars. The upper left graph depicts the possibility of breakthrough to originate from transmural conduction. The upper right graph depicts the likelihood of breakthroughs to originate from ectopic focal activity.

The two lower graphs depict the same analysis carried out for breakthrough events during application of flecainide in the same animals on the same location. 
Table 1 shows a direct comparison of the breakthrough rates in events/s before and after flecainide. For this analysis, a minimal transmural conduction velocity of $20 \mathrm{~cm} / \mathrm{s}$ and a maximal distance between source and site of breakthrough of $8 \mathrm{~mm}$ (grid size $5 \times 5$ electrodes) was assumed. Breakthroughs putatively due to transmural conduction were reduced by $78 \%$ in the $3 \mathrm{WAF}$ group and by $83 \%$ in the $6 \mathrm{mAF}$ group. Breakthroughs putatively due to ectopic focal discharges were reduced even more, by $94 \%$ in the $3 \mathrm{WAF}$ group and by $90 \%$ in the $6 \mathrm{mAF}$ group.

\begin{tabular}{|c|c|c|c|c|c|c|c|}
\hline & $\begin{array}{c}\text { aAF } \\
(n=7)\end{array}$ & $\begin{array}{l}3 w A F \\
(n=4)\end{array}$ & $\begin{array}{l}3 \text { wkAF } \\
+ \text { Flec } \\
(n=4)\end{array}$ & $\begin{array}{l}\% \text { change } \\
\text { after } \\
\text { flecainide }\end{array}$ & $\begin{array}{c}6 \mathrm{mAF} \\
(\mathrm{n}=4)\end{array}$ & $\begin{array}{l}6 \mathrm{mAF} \\
+\mathrm{Flec} \\
(\mathrm{n}=4)\end{array}$ & $\begin{array}{c}\% \text { change } \\
\text { after } \\
\text { flecainide }\end{array}$ \\
\hline $\begin{array}{c}\text { Entire breakthrough } \\
\text { population } \\
\text { (breakthrough/s) }\end{array}$ & $0.57 \pm 0.06$ & $3.20 \pm 0.55$ & $0.75 \pm 0.20$ & $-77 \%$ & $3.89 \pm 0.10$ & $0.83 \pm 0.14$ & $-79 \%$ \\
\hline $\begin{array}{c}\text { Presumably } \\
\text { Transmural } \\
\text { Conduction } \\
\text { (breakthrough/s) }\end{array}$ & $0.49 \pm 0.05$ & $2.75 \pm 0.47$ & $0.62 \pm 0.16$ & $-78 \%$ & $3.53 \pm 0.09$ & $0.63 \pm 0.10$ & $-83 \%$ \\
\hline $\begin{array}{l}\text { Presumably } \\
\text { Ectopc Focal } \\
\text { Discharges } \\
\text { (breakthrough/s) }\end{array}$ & $0.03 \pm 0.003$ & $0.86 \pm 0.14$ & $0.06 \pm 0.01$ & $-94 \%$ & $0.50 \pm 0.01$ & $0.05 \pm 0.008$ & $-90 \%$ \\
\hline
\end{tabular}

Table 1: Breakthrough rate (events/s) before and after flecainide administration. The breakthrough rates were higher with longer AF duration and significantly lower after flecainide administration. Please note that there is some overlap between the breakthroughs presumably caused by transmural conduction and the breakthroughs presumably caused by ectopic focal discharges as some breakthroughs meet the criteria for both transmural conduction as well as for ectopic focal discharges $(<15 \%$ of all breakthroughs). This explains why the sum of breakthroughs explainable by transmural conduction and those explainable by focal ectopic discharges is a little higher than the total population of breakthroughs. 


\section{Discussion}

This study demonstrates that flecainide reduces endo-epicardial dissociation of electrical activity and the incidence of transmural conduction (breakthrough) during AF. It also supports the hypothesis that flecainide inhibits ectopic focal discharges during AF. Both mechanisms might contribute to the antiarrhythmic effect of flecainide.

\section{Previous studies on the antiarrhythmic effect of flecainide}

Despite the fact that the effects of flecainide on atrial conduction and its effects on AF have been extensively studied the actual mechanism by which flecainide cardioverts AF is still unknown. Wijffels et al. showed that the antiarrhythmic effect of flecainide was associated with a widening of the excitable gap during AF. ${ }^{16}$ In the goat model of AF, they demonstrated that the 'wavelength' of fibrillation waves (atrial refractory period $\mathrm{x}$ conduction velocity) was shortened by flecainide, which in itself would favor the persistence of AF. However, they found that cardioversion of AF by a variety of antiarrhythmic compounds (including flecainide) always went along with widening of the temporal excitable gap (difference between AF cycle length and atrial refractoriness during $A F$ ) despite very different effects on refractory period and conduction velocity. In a later study, Danse et al. demonstrated in preparations of rabbit ventricles that flecainide caused conduction block preferentially at pivot points, thereby widening the excitable gap in the returning limb of a wave conducting along a line of block. ${ }^{13,17}$ If pivoting becomes less likely during $A F$, fibrillation waves might more likely propagate towards the border of the atria where they do not encounter excitable tissue anymore. 
This hypothesis is somewhat supported by a study of Kneller et al.. These authors used Langendorff perfused rabbit hearts with acetylcholine-induced AF and a mathematical model (Ramirez-Nattel-Courtemanche model) and proposed 3 major mechanisms by which flecainide could possibly cardiovert AF driven by rotors: (1) enlargement of the center of rotation beyond the capacity of the computational substrate, (2) decreased anchoring of rotors to functional obstacles, and (3) reduction of secondary wavelets that could provide new primary rotors. ${ }^{18}$ As we did not observe rotors in our model we cannot directly comment on these findings but inhibition of conduction at pivot points shows clear mechanistic parallels with enlargement of the core and less effective anchoring of rotors. In this sense, this study supports the concept of inhibition of conduction at pivot points and collision of waves with the boundary of the excitable tissue mass.

The loss of efficacy of flecainide to cardiovert in long term AF was studied by Eijsbouts et al. in the goat model of AF. ${ }^{19}$ They demonstrated that the loss of efficacy was not due to a loss of sensitivity to the drug, as it still prolonged the AFCL and the QRS interval significantly even beyond the values of earlier (successful) cardioversions.

The reduced pivoting of wave fronts after flecainide administration observed by Danse et al. initially led us to the hypothesis that flecainide favors the formation of broader homogeneous wave fronts. ${ }^{17}$ However, the present data do not support this hypothesis. As expected, the wave size decreased and the number of waves simultaneously present within the mapped region increased with AF duration. However, while application of flecainide significantly decreased the number of waves simultaneously present, it did not influence average wave size. 
This finding demonstrates that prolongation of the $\mathrm{AFCl}$ and reduction of conduction velocity by flecainide does not necessarily go along with reduced complexity of the substrate for AF. This finding would further indicate, that the increase in wave size, the gain of territory finally leading to one single propagating wave and cardioversion, is a process that occurs only shortly before cardioversion and is not an effect that directly correlates with the reduction of conduction velocity and AFCL seen during flecainide administration.

\section{Flecainide reduced EED and incidence of breakthrough}

All the above-mentioned mapping studies describe effects of flecainide on atrial conduction in the two-dimensional plane. ${ }^{16,} 18$ In the present study we demonstrate an additional effect of flecainide on the three-dimensional characteristics of AF. EED, a phenomenon recently described by our group, significantly increases with $A F$ duration and correlates with AF stability. ${ }^{8}$ Here, we demonstrate that flecainide significantly reduced EED in goats after 3 weeks and 6 months of AF. Furthermore, flecainide strongly reduced the incidence of breakthroughs, which are believed to contribute to the perpetuation of AF by providing sources of new fibrillation waves within the epicardial as well as endocardial layer of the atrial wall.

It has been shown in human and canine myocardial bundles that class IC agents (propafenone and flecainide) have a more pronounced effect on longitudinal than on transverse conduction. ${ }^{20,21}$ Longitudinal conduction most likely is more relevant for conduction within the epicardial and endocardial layer, whereas the less affected transverse conduction should be more relevant for endo-epicardial conduction. 
Therefore, the relevant changes of conduction caused by flecainide appear to be the following: As flecainide slows down conduction, prolongs the AF cycle length and widens the excitable gap, the average recovery time of atrial myocytes and thus safety of conduction increases. This effect as such would tend to cause fusion of fibrillation waves resulting in both broadening of fibrillation waves within the epicardial and endocardial layer and endo-epicardial resynchronization. However, flecainide directly impedes conduction by the $\mathrm{Na}^{+}$-channel blockade. The preferential inhibition of longitudinal conduction by flecainide might have prevented broadening of waves within the epicardial and the endocardial layer while weaker inhibition of transverse conduction allowed for expansion of endocardial waves to the epicardial layer and vice versa thereby reducing EED.

Breakthroughs show similarities with pivot points because they (if due to transmural conduction) represent fibrillation waves with abrupt change of the direction of propagation. Due to the reduced excitability of the downstream cells, the source/sink mismatch at breakthrough sites might, in the presence of flecainide, more likely lead to conduction block and thus inhibition of breakthrough.

Based on the correlation between EED and breakthrough rate demonstrated in chapter 4 , one would expect a proportional reduction of the breakthrough rate and EED after administration of flecainide. Interestingly, the decrease in breakthrough incidence by flecainide was even more pronounced than the reduction of EED. This points towards an additional effect of flecainide beyond the reduction of EED, which might be the inhibition of ectopic focal discharges. 


\section{Does flecainide inhibit ectopic focal discharges during AF?}

Testing all individual breakthrough events before and during flecainide application for their plausibility to originate from transmural conduction or focal ectopic activity, we demonstrate that flecainide reduced the percentage of breakthrough putatively originating from focal ectopic discharge more effectively than events putatively originating from transmural conduction.

A mechanistic explanation how flecainide might reduce ectopic focal discharges was recently provided by Knollmann and coworkers. They studied the effect of flecainide on ryanodine receptor $\mathrm{Ca}^{2}$ release channels and demonstrated that flecainide specifically acts as a blocker of these channels in the open state, which leads to smaller and shorter elementary sarcoplasmic $\mathrm{Ca}^{2}$ release events ("sparks") and thereby reduced the spark mass. ${ }^{22-24}$ Reduced spark mass (time-amplitude integral) resulted in a lower likelihood of the generation of proarrhythmic $\mathrm{Ca}^{2+}$ waves which are required to trigger delayed afterdepolarisations and ectopic action potentials. ${ }^{25}$ In line with this, they demonstrated a significant reduction of triggered beats in myocytes of transgenic mice and report two clinical cases of patients with catecholinergic polymorphic ventricular tachycardia successfully treated with flecainide.

The existence of ectopic focal discharges during $A F$ is uncertain and debatable. ${ }^{5,26-}$ ${ }^{28}$ Several studies have demonstrated hyperphosphorylation of ryanodine receptors in the atria of $\mathrm{AF}$ animal models ${ }^{28-30}$ and patients with $A F^{31}$ resulting in enhanced and proarrhythmic leak of $\mathrm{Ca}^{2+}$ from the $\mathrm{SR}^{26,27}$ That these phenomena result in ectopic focal discharges during AF has been strongly suggested by several authors ${ }^{26-28}$ but has recently been challenged by others. ${ }^{5}$ 
Even more obscure than the sheer existence of ectopic focal discharges during $A F$ is a quantitatively relevant contribution of these cellular arrhythmogenic events to the perpetuation of AF. ${ }^{9}{ }^{25}$ Also the present study cannot provide evidence for the relevance of ectopic focal discharges for perpetuation of AF but supports the hypothesis that during $\mathrm{AF}$ ectopic focal discharges exist and that they are suppressed by flecainide.

We would like to point out that this finding is an interpretation based on the algorithm which identifies breakthroughs originating from focal ectopic discharges by a putative relation of 2 simultaneous endo-epicardial breakthroughs. An alternate explanation for the more specific reduction of these events by flecainide could be higher safety of conduction in case of single endo- or epicardial breakthroughs which would then be less easily suppressed by flecainide. Studies to verify the criterion of simultaneous endo-epicardial breakthroughs as a surrogate for ectopic focal discharges are currently undertaken. 


\section{Concluding Hypothesis}

Based on the results from this study, we hypothesize, that the reduction of breakthrough incidence by flecainide can be attributed to three different mechanisms:

1. reduction of EED, thereby reducing the conditio sine qua non for breakthroughs originating from transmural conduction,

2. inhibition of breakthroughs by preferential block of conduction at sites of breakthrough (in which an abrupt change in direction of conduction occurs, comparable to e 3D pivot point), and

3. inhibition of ectopic focal discharges.

The fact that despite reduction of dissociation, decreased incidence of breakthrough and prolongation of AFCL no cardioversion is achieved in $6 \mathrm{mAF}$ goats points towards the existence of other relevant factors involved in the development of a substrate for AF. Factors of interest are related to structural changes of the atrial myocardium and subject of further studies. 


\section{References}

1. Morganroth J. Encainide for ventricular arrhythmias: placebo-controlled and standard comparison trials. Am J Cardiol. 1986;58(5):74C-82C.

2. Preliminary report: effect of encainide and flecainide on mortality in a randomized trial of arrhythmia suppression after myocardial infarction. The Cardiac Arrhythmia Suppression Trial (CAST) Investigators. N Engl J Med. 1989;321(6):406-412.

3. Camm AJ, Kirchhof P, Lip GY, Schotten U, Savelieva I, Ernst S, Van Gelder IC, Al-Attar N, Hindricks G, Prendergast B, Heidbuchel H, Alfieri O, Angelini A, Atar D, Colonna P, De Caterina R, De Sutter J, Goette A, Gorenek B, Heldal M, Hohloser SH, Kolh P, Le Heuzey JY, Ponikowski P, Rutten FH. Guidelines for the management of atrial fibrillation: the Task Force for the Management of Atrial Fibrillation of the European Society of Cardiology (ESC). Eur Heart J. 2010;31(19):2369-2429.

4. de Groot NMS. Comparison of epicardial breakthrough of fibrillation waves between patients with acute and chronic atrial fibrillation. In: Mapping and Ablation of Atrial TachyArrhythmias. Maastricht: Fysiologie, Maastricht University; 2006.

5. de Groot NM, Houben RP, Smeets JL, Boersma E, Schotten U, Schalij MJ, Crijns $\mathrm{H}$, Allessie MA. Electropathological substrate of longstanding persistent atrial fibrillation in patients with structural heart disease: epicardial breakthrough. Circulation. 2010;122(17):1674-1682.

6. Konings KT, Kirchhof CJ, Smeets JR, Wellens HJ, Penn OC, Allessie MA. High-density mapping of electrically induced atrial fibrillation in humans. Circulation. 1994;89(4):1665-1680.

7. Allessie MA, de Groot NM, Houben RP, Schotten U, Boersma E, Smeets JL, Crijns HJ. Electropathological substrate of long-standing persistent atrial fibrillation in patients with structural heart disease: longitudinal dissociation. Circ Arrhythm Electrophysiol. 2010;3(6):606-615.

8. Eckstein J, Maesen B, Linz D, Zeemering S, van Hunnik A, Verheule S, Allessie M, Schotten U. Time course and mechanisms of endo-epicardial electrical dissociation during atrial fibrillation in the goat. Cardiovasc Res. 2011;89(4):816-824.

9. Schotten U, Verheule S, Kirchhof P, Goette A. Pathophysiological mechanisms of atrial fibrillation: a translational appraisal. Physiol Rev. 2011;91(1):265-325. 
10. Wijfels MC, Kirchhof CJ, Dorland R, Allessie MA. Atrial fibrillation begets atrial fibrillation. A study in awake chronically instrumented goats. Circulation. 1995;92(7):1954-1968.

11. Ausma J, Wijffels M, Thone F, Wouters L, Allessie M, Borgers M. Structural changes of atrial myocardium due to sustained atrial fibrillation in the goat. Circulation. 1997;96(9):3157-3163.

12. Verheule S, Tuyls E, van Hunnik A, Kuiper M, Schotten U, Allessie M. Fibrillatory conduction in the atrial free walls of goats in persistent and permanent atrial fibrillation. Circ Arrhythm Electrophysiol. 2010;3(6):590-599.

13. Eckstein J, Verheule S, de Groot N, Allessie M, Schotten U. Mechanisms of perpetuation of atrial fibrillation in chronically dilated atria. Prog Biophys $\mathrm{Mol}$ Biol. 2008;97(2-3):435-451.

14. Holm M, Johansson R, Brandt J, Luhrs C, Olsson SB. Epicardial right atrial free wall mapping in chronic atrial fibrillation. Documentation of repetitive activation with a focal spread--a hitherto unrecognised phenomenon in man. Eur Heart J. 1997;18(2):290-310.

15. Kanagaratnam $\mathrm{P}$, Kojodjojo $\mathrm{P}$, Peters NS. Electrophysiological abnormalities occur prior to the development of clinical episodes of atrial fibrillation: observations from human epicardial mapping. Pacing Clin Electrophysiol. 2008;31(4):443-453.

16. Wijfels MC, Dorland R, Mast F, Allessie MA. Widening of the excitable gap during pharmacological cardioversion of atrial fibrillation in the goat: effects of cibenzoline, hydroquinidine, flecainide, and d-sotalol. Circulation. 2000;102(2):260-267.

17. Danse PW, Garratt CJ, Allessie MA. Flecainide widens the excitable gap at pivot points of premature turning wavefronts in rabbit ventricular myocardium. J Cardiovasc Electrophysiol. 2001;12(9):1010-1017.

18. Kneller J, Kalifa J, Zou R, Zaitsev AV, Warren M, Berenfeld O, Vigmond EJ, Leon LJ, Nattel S, Jalife J. Mechanisms of atrial fibrillation termination by pure sodium channel blockade in an ionically-realistic mathematical model. Circ Res. 2005;96(5):e35-47.

19. Eijsbouts S, Ausma J, Blaauw Y, Schotten U, Duytschaever M, Allessie MA. Serial cardioversion by class IC Drugs during 4 months of persistent atrial fibrillation in the goat. J Cardiovasc Electrophysiol. 2006;17(6):648-654.

20. Spach MS, Kootsey JM. Relating the sodium current and conductance to the shape of transmembrane and extracellular potentials by simulation: effects of propagation boundaries. IEEE Trans Biomed Eng. 1985;32(10):743-755. 
21. Kondo T, Yamaki M, Kubota I, Tachibana H, Tomoike H. Electrophysiologic effects of sodium channel blockade on anisotropic conduction and conduction block in canine myocardium: preferential slowing of longitudinal conduction by flecainide versus disopyramide or lidocaine. $J$ Am Coll Cardiol. 1997;29(7):1639-1644.

22. Hwang HS, Hasdemir C, Laver D, Mehra D, Turhan K, Faggioni M, Yin H, Knollmann BC. Inhibition of Cardiac Ca2+ Release Channels (RyR2) Determines Efficacy of Class I Antiarrhythmic Drugs in Catecholaminergic Polymorphic Ventricular Tachycardia. Circ Arrhythm Electrophysiol. 2011.

23. Hilliard FA, Steele DS, Laver D, Yang Z, Le Marchand SJ, Chopra N, Piston DW, Huke S, Knollmann BC. Flecainide inhibits arrhythmogenic Ca2+ waves by open state block of ryanodine receptor $\mathrm{Ca} 2+$ release channels and reduction of Ca2+ spark mass. J Mol Cell Cardiol. 2010;48(2):293-301.

24. Watanabe H, Chopra N, Laver D, Hwang HS, Davies SS, Roach DE, Duff HJ, Roden DM, Wilde AA, Knollmann BC. Flecainide prevents catecholaminergic polymorphic ventricular tachycardia in mice and humans. Nat Med. 2009;15(4):380-383.

25. Greiser M, Lederer WJ, Schotten U. Alterations of atrial $\mathrm{Ca}(2+)$ handling as cause and consequence of atrial fibrillation. Cardiovasc Res. 2011;89(4):722733.

26. Hove-Madsen L, Llach A, Bayes-Genis A, Roura S, Rodriguez Font E, Aris A, Cinca J. Atrial fibrillation is associated with increased spontaneous calcium release from the sarcoplasmic reticulum in human atrial myocytes. Circulation. 2004;110(11):1358-1363.

27. Neef S, Dybkova N, Sossalla S, Ort KR, Fluschnik N, Neumann K, Seipelt R, Schondube FA, Hasenfuss G, Maier LS. CaMKII-dependent diastolic SR $\mathrm{Ca} 2+$ leak and elevated diastolic $\mathrm{Ca} 2+$ levels in right atrial myocardium of patients with atrial fibrillation. Circ Res. 2010;106(6):1134-1144.

28. Chelu MG, Sarma S, Sood S, Wang S, van Oort RJ, Skapura DG, Li N, Santonastasi M, Muller FU, Schmitz W, Schotten U, Anderson ME, Valderrabano M, Dobrev D, Wehrens XH. Calmodulin kinase II-mediated sarcoplasmic reticulum $\mathrm{Ca} 2+$ leak promotes atrial fibrillation in mice. J Clin Invest. 2009;119(7):1940-1951.

29. Greiser M, Neuberger HR, Harks E, El-Armouche A, Boknik P, de Haan S, Verheyen F, Verheule S, Schmitz W, Ravens U, Nattel S, Allessie MA, Dobrev D, Schotten U. Distinct contractile and molecular differences between two goat models of atrial dysfunction: AV block-induced atrial dilatation and atrial fibrillation. J Mol Cell Cardiol. 2009;46(3):385-394. 
30. Li D, Fareh S, Leung TK, Nattel S. Promotion of atrial fibrillation by heart failure in dogs: atrial remodeling of a different sort. Circulation. 1999;100(1):87-95.

31. El-Armouche A, Boknik P, Eschenhagen T, Carrier L, Knaut M, Ravens U, Dobrev D. Molecular determinants of altered $\mathrm{Ca} 2+$ handling in human chronic atrial fibrillation. Circulation. 2006;114(7):670-680. 



\title{
Chapter 6
}

\section{Mapping of Atrial Fibrillation -}

\section{Basic Research and Clinical Applications}

\author{
Jens Eckstein, $M D^{1}$, Michael Kühne, $M D^{2}$, \\ Stefan Osswald, $\mathrm{MD}^{2}$, Ulrich Schotten, MD, $\mathrm{PhD}^{1}$ \\ 1: Cardiovascular Research Institute, Department of Physiology, University Maastricht, Maastricht, The Netherlands, \\ 2: University Hospital Basel, Division of Cardiology, Basel, Switzerland
}

Published in: Swiss Med Wkly. 2009;139(35-36):496-504 


\section{Abstract}

Despite five decades of intensive research, mechanisms initiating and stabilizing atrial fibrillation (AF) are still not fully understood. Nevertheless, mapping studies, next to clinical trials and research on cellular electrophysiology, have provided key information that led to a much more profound understanding of the arrhythmia.

Contact mapping using multi-electrode arrays (MEAs) is the gold standard for high resolution mapping in basic research and clinical trials and continuously contributes to a better description of mechanisms perpetuating AF. It thereby provides information needed to target and test new pharmacological and interventional treatment options for AF therapy and to evaluate established ones, which were often implemented based on purely empirical assumptions.

High resolution contact mapping studies are performed in patients undergoing cardiac surgery for basic research purposes to evaluate to which extent data derived from animal models of $A F$ is comparable to data recorded in humans. The goal of these research projects is to develop algorithms, which allow the identification and staging of the arrhythmogenic substrate. This information should then help to guide surgical therapy when applicable, or individualize treatment strategy involving catheter ablation, antiarrhythmic drug therapy or simply a rate control strategy.

Mapping techniques used in the catheter laboratory by interventional electrophysiologists represent a valuable tool for exact localization of catheters and the points of interest for ablation. These techniques integrate data on individual anatomy (derived from CT scan or intracardiac ultrasound), local intracardiac electrograms (recorded point by point with a catheter) and the exact spatial position of the catheter. 
While mapping techniques used with electrophysiological studies and ablations in patients are highly useful tool to optimize and document ablation results and significantly reduce fluoroscopy time, they fail to display the complexity of atrial activation during AF. This is mainly due to a limited number of simultaneously recorded electrograms and a low spatial resolution which is sufficient for its clinical use. At present high resolution mapping of AF in patients is only feasible during cardiac surgery. Endocardial catheter based systems that have recently become available have to be further evaluated but might provide an option in this setting in the near future. 


\section{Introduction}

Atrial Fibrillation $(\mathrm{AF})$ is a progressive disease frequently transforming from paroxysmal to persistent AF. Mechanisms underlying this process of increased stabilization have been subject of extensive research. Major contributions for the understanding of the circumstances initiating and perpetuating the arrhythmia derive from mapping studies in patients and animal models of $\mathrm{AF}^{1}$.

The term "mapping" is used for procedures recording local electrical signals at sites of interest on the endocardium or epicardium and then allocating them to a spatial matrix reflecting the mapping area. The following article will provide an overview of mapping techniques used for different purposes in basic research and as clinical tools.

Early mapping studies were performed using custom made multi-electrode arrays (MEAs) recording between 64 and 256 simultaneous local epicardial electrograms. For electrogram acquisition MEAs were placed on atria of patients undergoing cardiac surgery ${ }^{2}$, animals in open chest experiments ${ }^{3}$ or Langendorff perfused hearts ${ }^{1}$. The signals were then allocated to a matrix reflecting the location of the corresponding electrode in the MEA. By interconnecting neighboring points of simultaneous activation (Isochronal lines) the activation sequence of the atrial surface reflecting propagation of fibrillation waves can be visualized more easily. Examples of activation patterns of the atrial free wall are shown in figure 1. 

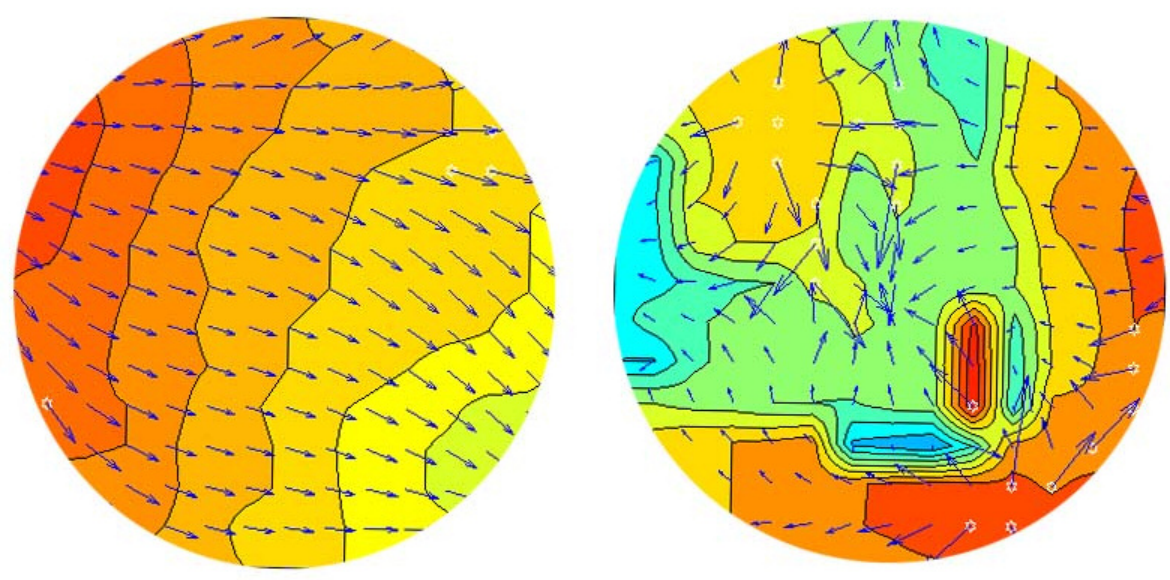

Figure 1: Activation time maps showing wave propagation over the atrial free wall. Isochronal lines indicate $5 \mathrm{~ms}$ time differences, earliest activation is in red, latest in blue. Arrows represent local direction of propagation. White stars mark sites of supra-normal local conduction velocities due to oblique activation. Diameter of the mapping area displayed is $48 \mathrm{~mm}$.

Left: Homogeneous fast propagation of a single wave during sinus rhythm.

Right: Complex activation pattern during $A F$ with a broad wave coming from the right and lower side, a second wave originating in the left upper quadrant leading to a "breakthrough" pattern with radial spread of activation and a number of smaller waves. The narrow spaces between some isochronal lines represent slow conduction, the wide spaces accordingly areas activated almost simultaneously. 
The left panel displays a single wave propagating through the mapping area during sinus rhythm. Broad spaces between isochronal lines and the homogeneous orientated arrows (indicating local direction of propagation) can be interpreted as fast homogeneous conduction. The right panel shows a complex activation pattern observed during AF. Pronounced inhomogeneity of conduction resulting in local crowding of isochronal lines (= slowing of conduction) is present. Further, spots with radial spread of activation originating within the mapping area can be recognized. These events are likely to be caused either by a preceding endocardial wave, "surfacing" and then propagating in the epicardial plane ("breakthrough") or by true local automaticity.

This type of AF mapping (Activation map) led to the description of different mechanisms, which could be responsible individually or in combination for the property of AF to sustain itself.

The first hypothesis describing a potential mechanism leading to sustained AF without the need for a driving source was published by Moe and coworkers in $1959^{4}$. They proposed the presence of multiple wavelets propagating through the atrium as potential mechanism for the self-sustaining nature of AF. 26 years later Allessie and coworkers were able to demonstrate wavelets using activation time maps of acetylcholine induced AF in isolated canine atria ${ }^{1}$. This was the first experimental demonstration of multiple wavelets propagating on the epicardial surface during AF. Konings et al. compared Allessie's findings in dogs to AF patterns in patients with Wolff-Parkinson-White syndrome undergoing cardiac surgery for accessory pathway interruption ${ }^{2}$. By mapping the right atrial free wall of these patients they could confirm the presence of multiple wavelets in pacing induced AF in humans. 
This was one of the first mapping studies to describe "breakthrough" events during AF in patients as a propagation pattern with radial spread of activation induced by either local automaticity or transmural activation of the epicardium from the endocardial bundle network. Since then breakthrough patterns (Figure 1, right panel) were described in multiple studies and are still part of the ongoing discussion about mechanisms initiating and maintaining AF.

In a later mapping study Konings et al. described causes underlying fractionation of electrograms and thereby contributed to the background of some targets of catheter ablation therapy referred to as complex fractionated atrial electrograms (CFAEs) ${ }^{5-7}$.

It is probably due to technical reasons and to the fact that the atrial wall is considerably thinner than the ventricular wall, that the majority of mapping studies used a two dimensional, predominantly epicardial, approach resulting in description of two dimensional patterns such as spiral waves, wavelets ${ }^{1}$, rotors $^{8}{ }^{9}$, multiple unstable reentrant circuits ${ }^{10}$ or foci ${ }^{11}$.

Although the third dimension of the atrial wall was frequently taken into account in the discussion about breakthroughs and foci, very few studies so far provided data on simultaneous endo-epicardial high-resolution mapping ${ }^{12}$. Considering the complexity of the atrial structure and the irregular pattern of fibrillation waves, insight in the three-dimensional organization of $\mathrm{AF}$ is needed in order to elucidate the origin of breakthrough activity and other possible mechanisms underlying the increasing stability of AF over time.

While high density contact mapping using MEAs or optical mapping techniques are at present mainly used for basic research purposes in patients and animals, catheter 
based mapping techniques have been established in recent years as valuable clinical tools for interventional electrophysiologists to facilitate complex electrophysiological procedures, increase anatomical accuracy and patient safety, and reduce fluoroscopy times.

Electroanatomic mapping systems integrate local electrograms recorded by the mapping and ablation catheter and the three-dimensional anatomy of the mapped cardiac chamber.

The purpose of this article is to give an overview of the current basic research and clinical applications used for mapping of AF. 


\section{Mapping sequence and parameters}

All mapping techniques described in the basic research and the clinical application section are based on the acquisition of local electrograms. A significant difference between direct contact mapping using MEAs or optical mapping and catheter based mapping techniques is the simultaneous versus the sequential fashion to record the electrograms. While simultaneous recording allows demonstration of continuous complex patterns of wave propagation sequential recording "only" represents a number of single snapshots. Still, in cases of more homogeneous and stable activation patters as present in atrial tachycardia or atrial flutter these snapshots can be connected to a sequence most likely representing the actual pattern of propagation.

This explains why sequential mapping fails to produce maps displaying the activation sequence (Activation-map) of complex propagation patterns during AF. All the following parameters can be obtained from both approaches:

The local amplitude of electrograms (Voltage-map) is a parameter used to identify areas of scaring and fibrosis (low voltage) caused by past interventions or degeneration due to intrinsic degenerative processes.

The local AF cycle length (Frequency-map) provides information about sites possibly "driving" the arrhythmia (short cycle length) and bystanders (long cycle length).

The degree of fractionation of local electrograms (Fractionation-map) is used to locate targets for ablation based on the hypothesis that sites with a high degree of fractionation (CFAEs) are responsible for the perpetuation on the fibrillatory process $^{6}$. 


\section{Mapping of atrial fibrillation - Present basic research}

Several animal models ranging from mice to dogs and goats are used for mapping of AF and atrial arrhythmias. Even though caution is required when extrapolating data derived from animals studies to human pathologies key findings that influenced AF research and therapy in humans derive from animal models of AF. Examples to be named are the demonstration of multiple fibrillation wavelets ${ }^{1}$, electrical remodeling 13 and fractionation of fibrillation electrograms ${ }^{5}$. Although there are models presenting with spontaneous initiation AF we still lack an animal model to study the role of PV triggers which play a highly important role in the initiation of paroxysmal AF in humans ${ }^{11}$. Next to extensive studies aiming at a better understanding of the circumstances contributing to the progressive time course of AF, recent and ongoing studies evaluate the effects of established or newly developed drugs for prevention or conversion of AF. In this context mapping experiments allow to study the changes in AF morphology (AF cycle length, number of waves present, width of waves, conduction velocity, electrogram changes) preceding cardioversion or preventing it. The majority of human high-density mapping studies for basic research purposes is performed in patients undergoing cardiac surgery. At present, this data is correlated with findings derived from animal models to develop algorithms for "staging" of human AF based on individual mapping data.

Three major fields of mapping in basic research will be discussed in the following section: Contact mapping using MEAs in animal models, contact mapping using MEAs in patients, and optical mapping in animal models. 


\section{Contact mapping using MEAs in animal models of AF}

Direct contact of the MEA and the atrial tissue is the gold standard for high resolution mapping of AF. Depending on the principal research question addressed and the technical possibilities, a standard mapping setup usually consists of a MEA (commonly 256 electrodes), a mapping amplifier and a computer for data acquisition and analysis. Although the required hardware is commercially available many systems are custom made to address specific questions.

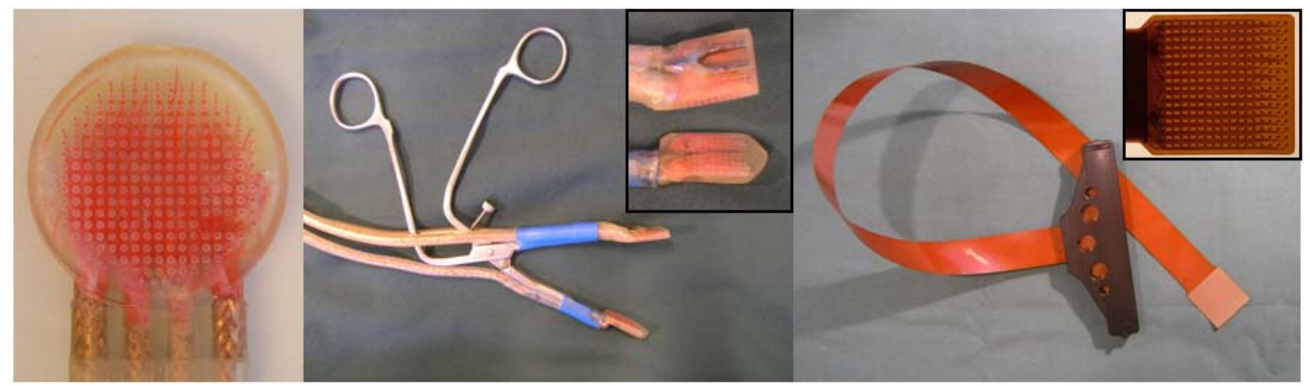

Figure 2: Custom made multi electrode mapping arrays (MEAs):

Left: "Spoon", 256 electrodes, spatial resolution 2.4mm, used for mapping of the atrial free wall in animals and humans.

Middle: "Crocodile", 246 electrodes, spatial resolution 1.6mm, used for simultaneous endo-epicardial mapping of the atrial free wall in animals. By inserting the lower part of the clamp into the atrial cavity, the atrial wall between the two MEAs can be mapped simultaneous endo- and epicardially.

Right: Single use Flex-MEA, 256 electrodes, spatial resolution $1.5 \mathrm{~mm}$, used for high-resolution epicardial contact mapping in patients during cardiac surgery. 
Three examples of custom-made MEAs are given in figure 2. The left panel shows a "Spoon electrode" which is used in animals and humans, with a diameter of $48 \mathrm{~mm}$, consisting of 256 single electrodes. This device allows to record simultaneous electrograms of the entire right or left atrial free wall during AF (as shown in figure 1). The mid panel shows a recently developed mapping tool ("Crocodile electrode") used for simultaneous high-resolution endo-epicardial mapping (as shown in figure 3). First results using this technique in the goat model of AF have recently been published and provide new insight in the three-dimensional organization of $\mathrm{AF}^{14}$. With this tool we could demonstrate that, while there is simultaneous activation of the endocardial bundle network and the subepicardial layer during sinus rhythm, there is considerable endo-epicardial dissociation of activation during AF (Figure 3). These findings were fully in line with data published by Schuessler et al demonstrating for the first time endo-epicardial dissociation of electric activity during acetylcholine induced $A F$ in perfused preparations of canine atria using high density mapping ${ }^{12}$. In our goat model of long term AF we were able to demonstrate that endo-epicardial dissociation is present in vivo and that it significantly increases if $A F$ is maintained for up to six months duration. This circumstance is likely to contribute to the further increasing stability of AF after electric remodeling has occurred ${ }^{15}$. 


\section{Epicardial}

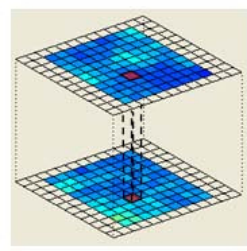

Endocardial
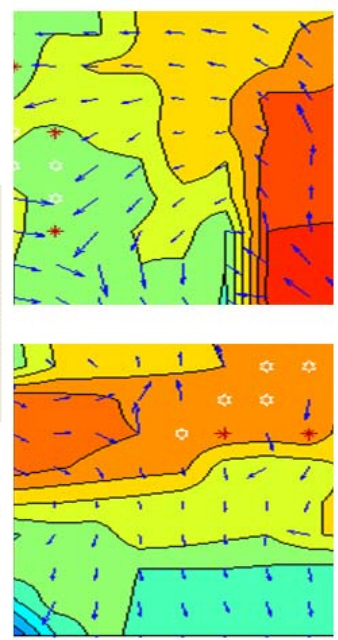

Figure 3: Isochronal map of an endoepicardial recording of $A F$ using the "crocodile" electrode in a goat. Both maps are recorded simultaneous at exactly opposing sites on the epicardial and the endocardial surface respectively. Isochronal lines indicate $5 m s$ time differences, earliest activation is in red, latest in blue. Arrows represent local

direction of propagation. White stars mark sites of supra-normal local conduction velocities due to oblique activation. Comparing the endocardial with the epicardial activation pattern reveals, that during $A F$ electrical dissociation in time (delta activation time lower right corner $=35 \mathrm{~ms}$ ) and direction of propagation (difference in direction of propagation upper left corner $=180^{\circ}$ ) between the subepicardial layer and the endocardial bundle network is present.

By demonstrating the presence of endo-epicardial dissociation during AF we further confirmed a prerequisite condition for the occurrence of breakthrough events. These events present as focal activity in conventional two dimensional mapping and are an important subject in the ongoing discussion about the mechanisms initiating and perpetuating AF. A main question of this research focuses on the relevance of these events for perpetuation of $\mathrm{AF}$ and their actual origin as this could be either be truly local activity or part of a fibrillation wave propagating on the opposite layer of the atrial wall and emerging at this very site causing a "breakthrough" pattern. 
The differentiation of these two mechanisms appears to be important because it could contribute to the understanding of the time course underlying AF and help to tailor therapeutic interventions.

\section{Contact mapping using MEAs in patients undergoing cardiac}

\section{surgery}

With new tools for surgical AF therapy being available and further refinement of established surgical approaches the option of contact mapping along with these surgical procedures provides an valuable tool to compare former findings from animal models to those in humans ${ }^{16-18}$. Along with this, mapping specific parameters are evaluated for their use to guide ablation therapy and/or contribute to the decision making in choosing the appropriate therapeutic approach based on individual mapping data.

An example of a newly developed single use high-density mapping MEA for intraoperative mapping in humans is displayed in the right panel of figure 2 . This MEA is mounted on a positioning tool and placed at predefined locations of the epicardial atrial surface to simultaneously record up to 256 local electrograms. It is obvious that signals can only be obtained when the heart is still beating. Therefore mapping has to be performed before cardioplegia is started or along with operations on the beating heart ("off pump"). With minimally invasive procedures for surgical AF therapy being established, the use of Flex-MEAs in the context of this approach appears to be feasible too ${ }^{18}$. 
As the field of surgical interventions for AF therapy is expanding, direct access to the epicardial surface providing the option of high-density mapping might represent a relevant advantage of this approach in terms of identifying targets and direct evaluation of the ablation procedure ${ }^{19}$.

\section{Optical mapping in animal models of AF}

Optical mapping represents an alternative mapping technique in basic research. It can be performed in isolated hearts or tissue preparations that are perfused with standard Tyrode solution. Together with the perfusate, a bolus of voltage sensitive dye (e.g. Di 4 ANEPPS) is applied which then integrates into the cellular membrane. The fluorescence of this dye is linearly proportional to the membrane potential. The emitted fluoroscopic signal thus reflects an ensemble action potential. Therefore, this signal can be interpreted in terms of measuring not only the activation time but also the action potential duration and the diastolic interval. Analyzing sequential frames, which can be acquired as fast as $10.000 /$ second, the spread of activation over the entire mapping area can be visualized as a movie (Figure 4). Spatial resolution of this technique is dependent on the optical hardware but can be as high as $100 \mathrm{~mm}$. Despite superior spatial and temporal resolution, optical mapping involves a number of relevant disadvantages: first, the preparation has to be flattened out to provide equal distance of the mapped tissue to the optics. Second, only the upper $200 \mu \mathrm{m}$ of the tissue contribute to the emitted signals and third, an excitation contraction uncoupler (e.g. BDM, Cylochalase or Blebistatin) has to be infused to prevent movement artifacts due to contraction of the tissue. 

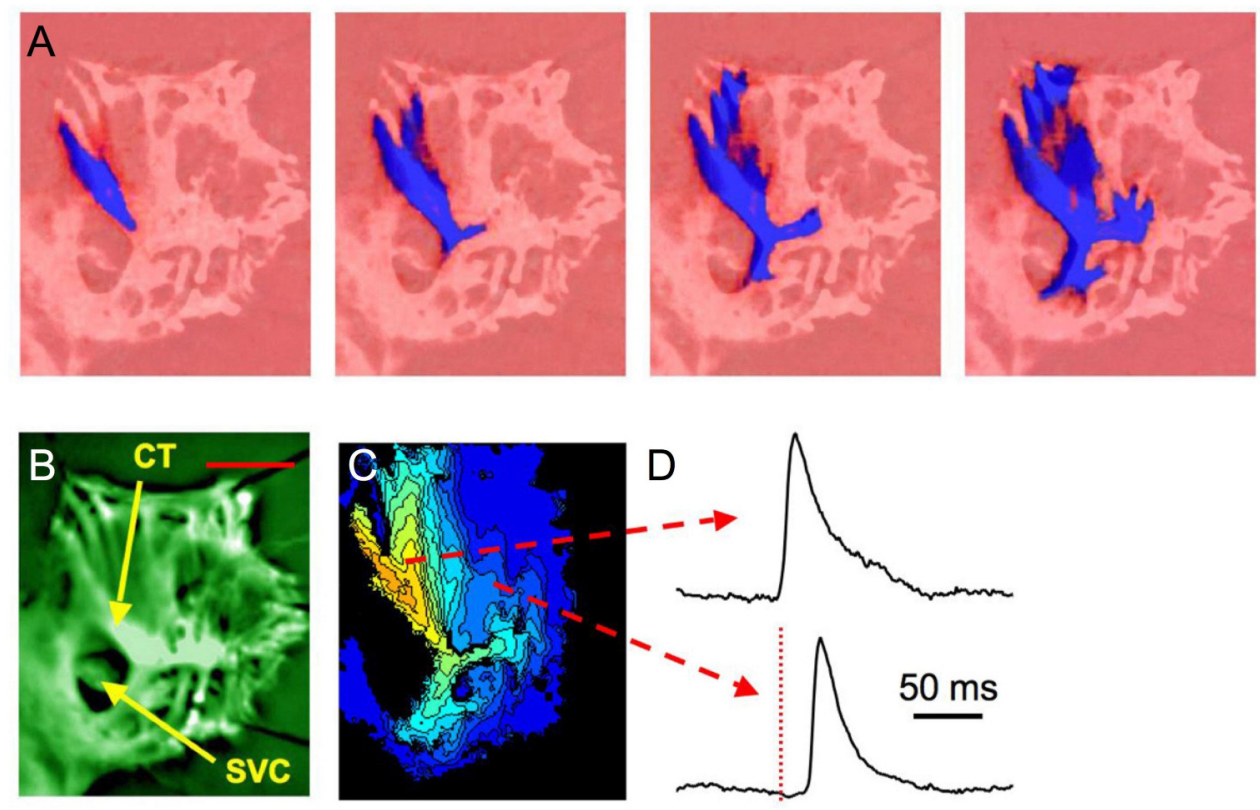

Figure 4: Endocardial optical mapping activation pattern during sinus rhythm in the right atrium of a rabbit. A) Consecutive fames separated by $4 m s$ B) Endocardial bundle structure of the right atrium, CT=christa terminalis, SVC= superior vena cava, C) Optical activation map in this preparation, red=early, blue=late, D) Optical action potential in the CT (upper trace) and the thin layer between the trabeculae (lower trace)

Considering these factors, it is obvious that this method cannot be used for human mapping but nevertheless provides an attractive alternative method for experimental studies. 


\section{Mapping of atrial fibrillation - Present clinical applications}

Present clinical mapping applications used by interventional electrophysiologists serve the purpose of finding goals for catheter ablation. This is achieved by analysis of local electrograms guided by conventional fluoroscopy and - when using an electroanatomic mapping system - by obtaining three-dimensional anatomical information and creating a reconstruction of a cardiac chamber (e.g. left atrium) to maximize anatomical accuracy (and patient safety) and to minimize radiation exposure.

Mapping in the clinical setting means to determine where an arrhythmia is originating from in case of a focal tachycardia (e.g. pulmonary vein tachycardia) or to identify the course of the circuit in case of a reentry tachycardia (e.g. left atrial macro-reentry tachycardia). In contrast to high-resolution contact mapping in research applications, clinical mapping tools used in conjunction with a standard focal mapping catheter result in a relatively low resolution and rely on sequential rather than simultaneous recordings of electrical activity. However, specific mapping catheters allowing simultaneous recordings of electrical activity from different poles are available for contact and noncontact mapping in interventional electrophysiology. An example of a multipolar ring catheter used for pulmonary vein recordings is shown in Figure 5 and its use is further explained below. 


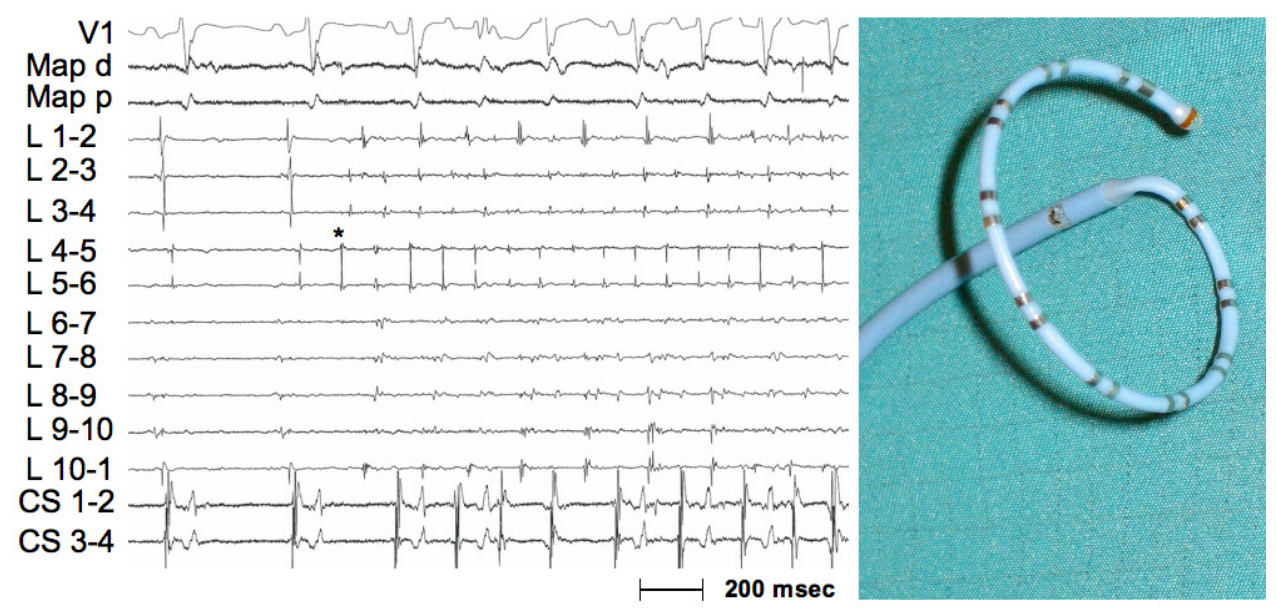

Figure 5: Left: Initiation of AF during Isuprel infusion (10 $\mathrm{mcg} / \mathrm{min}$.). Shown are one surface lead (V1), and intracardiac electrograms recording the distal and proximal poles of the mapping and ablation catheter (Map d, Map p) positioned on the ventricular side of the mitral anulus, the multipolar ring catheter ( $L$ 1-2 (distal) to $L$ 10-1 (proximal)) in the right superior pulmonary vein, and the coronary sinus catheter (CS 1,2 (distal) to CS 9,10 (proximal)). The first two beats are sinus beats with passive activation of the pulmonary vein. Then, rapid electrical activity (cycle length $100 \mathrm{~ms}$ ) originating from the pulmonary vein with the earliest local endocardial activation recorded on L 4-5 (asterisk) initiates AF. Right: Example of a multipolar ring catheter with a variable diameter (LASSO 2515, Biosense Webster Inc. Diamond Bar, CA, USA) for mapping of the pulmonary veins. 
The importance of the pulmonary veins as a source of ectopic beats and their role in triggering and maintaining atrial fibrillation was discovered more than ten years ago ${ }^{11}$. Based on these findings catheter ablation strategies were developed with the goal of electrically isolating the pulmonary veins from the left atrium ${ }^{20,21}$. Further studies involving mapping techniques showed that not only there is a dynamic interplay between the pulmonary veins and the left atrium, but also that there are mechanisms apart from the pulmonary veins in both atria that are relevant for the perpetuation of AF. This is particularly important in patients with the persistent and permanent form of the arrhythmia. These findings led to treatment strategies targeting sites in the left atrium displaying specific types of local electrograms (complex fractionated atrial electrograms $=$ CFAE, figure 6), approaches including linear lesion sets in the left atrium with the goal of modifying the arrhythmogenic substrate and approaches targeting ganglionic plexi within the intrinsic cardiac autonomic system ${ }^{6,7,22,23}$. The strategy of targeting CFAEs was based on findings of earlier mapping studies postulating that local electrograms are fractionated in case multiple waves are simultaneously present. CFAEs can occur due to complex fibrillatory conduction in areas with conduction disturbances and are considered to contribute to the maintenance of $\mathrm{AF}^{5}$. This hypothesis was supported by the work of Nademanee and coworkers who targeted sites of most extensive electrogram fractionation after creating electroanatomic maps of both atria ${ }^{6}$. Targeting these sites (plus additional ibutilide treatment in some patients) resulted in a high rate of conversion to sinus rhythm. However, a recent study showed that additional ablation of CFAEs after pulmonary vein isolation did not improve clinical outcomes in patients with longlasting persistent $\mathrm{AF}^{24}$. 


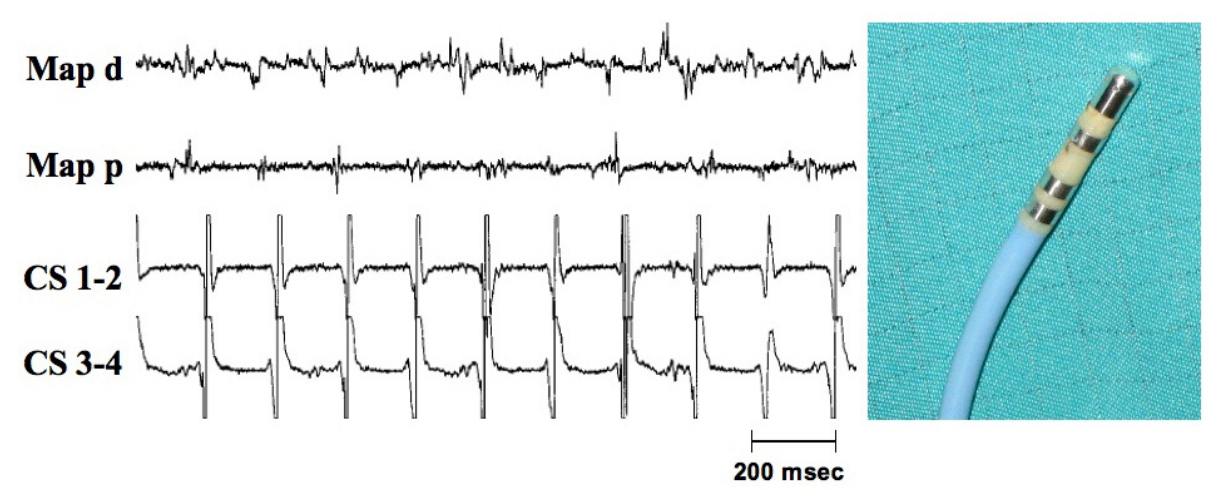

Figure 6: Left: Example of a complex fractionated atrial electrogram (CFAE) recorded with the distal pole of the mapping catheter (Map d) at a septal site in the left atrium. Note the high degree of fractionation and short cycle length of the electrogram on Map d. In contrast, the electrogram in the coronary sinus (CS) is well organized with a relatively stable cycle length of approximately 180 msec. Right: A 3.5-mm irrigated-tip catheter (Thermocool, Biosense Webster Inc. Diamond Bar, CA, USA) used for mapping (and ablation).

Mapping and ablation procedures in patients with $\mathrm{AF}$ require accurate navigation in the left atrium. This can be accomplished using standard fluoroscopy or an electroanatomic mapping system, which combines the anatomical and electrical information that is gained during point-by-point mapping with the catheter and allows the construction of a three-dimensional shell of the left atrium or any cardiac chamber. 


\section{Anatomic mapping}

An integral part of an electroanatomic mapping system is the functionality of localization of a catheter in three dimensions without the need for fluoroscopy. There are two different electroanatomic mapping systems that are widely used in clinical practice. One is an electrical impedance mapping system (NavX, St. Jude Medical Inc., Minneapolis, MN, USA) using voltage and impedance for localization, the second one is a magnetic mapping system (CARTO, Biosense Webster Inc. Diamond Bar, CA, USA) using magnetic fields. The two systems are briefly explained in the following two paragraphs.

When using a magnetic electroanatomic mapping system (CARTO), the localization is based on the principle that a coil in a magnetic field generates an electrical current

25 . The current is dependent on the strength of the magnetic field and the orientation of the coil. The magnetic field (three fields, $5 \times 10^{-6}$ Tesla) is produced by a location pad which is placed on the patient's back. The tip of the mapping catheter contains a sensor with three coils oriented perpendicular to each other. The sensor measures the magnetic field based on the electrical current that is induced. Based on the fact that the strength of the magnetic field decays with increasing distance from the source, the distance from the three magnetic fields generated by the location pad can be measured. This allows accurate localization of the catheter in space. Each location of the mapping catheter can then be registered on a three-dimensional map and a shell of the anatomical structure that is mapped (e.g. left atrium) can be constructed. Catheter positions and contact with the endocardium can occasionally be verified by fluoroscopy, local electrograms and in some laboratories intracardiac ultrasound. Anatomical landmarks (e.g. pulmonary vein ostium, esophagus) can be 
tagged on the map. The accuracy of the map depends on the number of sampled points and the distance between them. Patient movement during the mapping procedure and respiration and cardiac motion are limitations to the accuracy of the map.

Electrical impedance mapping systems (NavX) use three pairs of surface electrodes placed on the patient's chest in three axes (perpendicular to each other). Using these three electrode pairs, the chest is placed in an electrical field with a current of $350 \mu \mathrm{A}$ at a frequency of $5.7 \mathrm{kHz}$. This creates a transthoracic voltage gradient in the three axes. As a different frequency is used for each electrode pair filtering recorded signals (from electrodes of a catheter) for these frequencies allows the calculation of the three-dimensional position of the catheter and all its poles in case of a multipolar catheter. The calculation of the catheter position is based on the fact that the voltage and impedance changes measured by the catheter are proportional to the distance from the surface electrodes. An advantage of the electrical impedance mapping system is that more than one catheter and all poles of a catheter (in case of a multipolar catheter) can be visualized simultaneously and that all poles can be used to create the three-dimensional geometry of a cardiac chamber. However, changes in thoracic impedance may adversely affect the accuracy of the maps with this system.

With the aim of further improving the anatomic accuracy of the maps, integration of three-dimensional reconstructions of images obtained non-invasively by computed tomography (CT), magnetic resonance imaging (MRI) and of images acquired with intracardiac ultrasound during the procedure (before transseptal puncture) has become available. Image integration is performed by defining landmark points on the 
CT or MRI reconstruction of the left atrium followed by merging the CT or MRI image with the anatomical map that has been constructed using the mapping catheter (Figure 7). A limitation of this technique is that the CT or MRI image is not acquired real-time, but usually on the day before the procedure, and differences in volume status (open irrigated-tip catheter, anesthesia) can result in significant differences in left atrial volume.

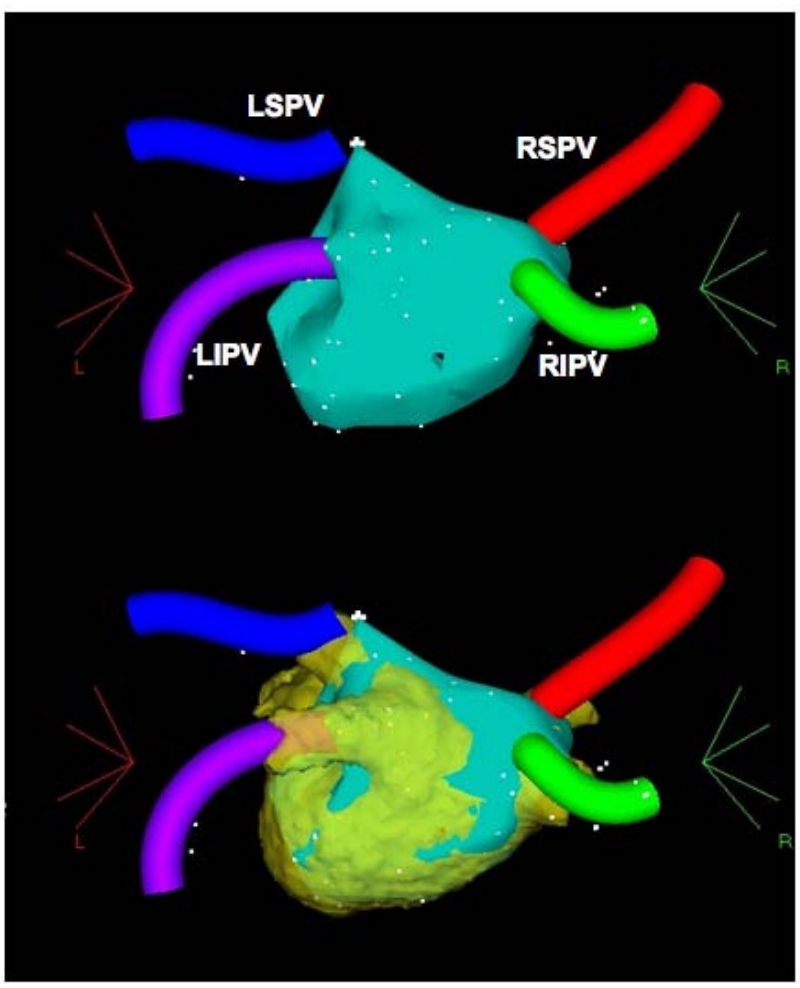

Figure 7: Top: Postero-anterior view of a three-dimensional reconstruction (CARTO map) of the left atrium (in green) acquired by catheter-based mapping. The pulmonary veins are tagged and drawn as isodiametric tubes. (LSPV / $L I P V=$ left superior / inferior pulmonary vein; RSPV / RIPV = right superior / inferior pulmonary vein)

Bottom: Merging of the CARTO map with a three-dimensional reconstruction of the left atrium obtained by computed tomography (shown in yellow) by registration of landmark points at the pulmonary vein ostia. 
Currently available image integration with intracardiac echo is based on twodimensional ultrasound requiring "slice-by-slice" image acquisition for reconstruction of the left atrial shell, but has the advantage of real-time visualization of the cardiac structures and the position of the catheter. Applications using three-dimensional intracardiac echocardiography are under development.

\section{Electrical mapping}

In order to achieve complete electrical isolation of the pulmonary veins during AF ablation procedures the operator needs a reliable tool to identify the electrical connections between the pulmonary veins and the left atrium. These electrical connections are preferably demonstrated with a multipolar circular mapping catheter with the shape of a Lasso (figure 5). The electrical information gained by electrogram recordings from the circular mapping catheter not only shows the presence and the exact anatomical site of an electrical connection between the pulmonary vein and the left atrium, but also provides information on the electrical activity of the pulmonary vein with regard to triggering or driving AF. Furthermore, the recordings from the circular mapping catheter are the basis for demonstration of complete pulmonary vein isolation after ablation. If complete conduction block between the pulmonary vein and the atrium is present (=isolation), the electrical activity of the left atrium will no longer conduct into the pulmonary vein, and therefore a circular mapping catheter placed distal to the line of block should no longer record local electrical signals (unless they originate in the pulmonary veins).

The pulmonary veins certainly play a pivotal role in paroxysmal AF, but treatment strategies exclusively focusing on pulmonary vein isolation appear to have significant 
shortcomings especially in patients with persistent AF. This is due to the fact that $A F$ and especially maintenance of AF is not only dependent on the pulmonary veins, but also on the (arrhythmogenic) substrate which is predominantly located in the remodeled left atrium. As mentioned earlier specific types of local electrograms recorded with the mapping catheter (complex fractionated atrial electrograms $=$ CFAEs) have been identified as targets for ablation (Figure 6). CFAEs are low voltage electrograms that are fractionated and rapid (cycle length $<120 \mathrm{~ms}$ ) and catheter ablation at sites with CFAEs has resulted in successful treatment of $A F$ in a number of patients ${ }^{6}$.

\section{Electroanatomic activation mapping}

When sampling points in the left atrium using a mapping catheter, not only anatomical (localization) and electrical (voltage, impedance) information, but also data on the timing of electrical activation of a particular site can be gained. When combining the information on a three-dimensional electroanatomic map, the timing of the electrical activation of all sampled points can be color-coded with early activation coded in red, later activation coded in green, and very late activation coded in blue and purple. In clinical practice without high-density mapping, this results in demonstration of chaotic electrical activity of the left atrium in AF, but is particularly helpful for mapping of focal atrial tachycardias and macro-reentrant atrial flutter. The latter arrhythmia is not infrequent after catheter ablation of atrial fibrillation and is usually mapped using activation mapping, entrainment mapping (measuring postpacing intervals), or both. 
An electroanatomic activation map in a patient with persistent atrial fibrillation and an activation map in a patient a focal atrial tachycardia are shown in figure 8.

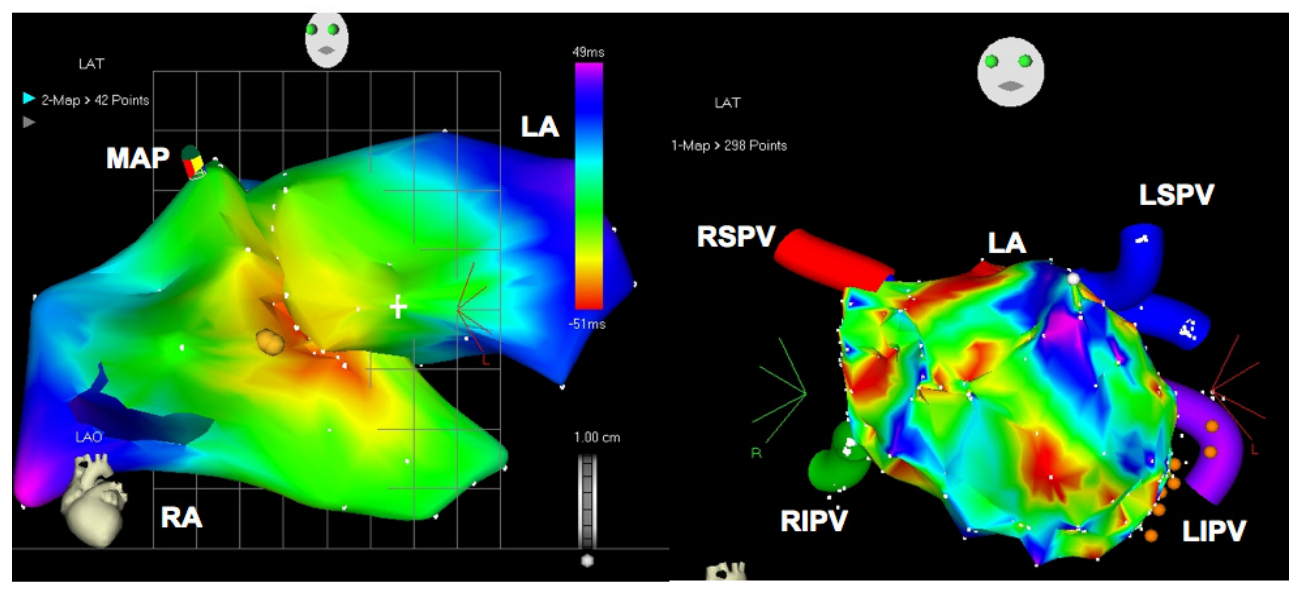

Figure 8: Left: Left anterior oblique view of a three-dimensional electroanatomic activation map of the right atrium $(R A)$ and the left atrium $(L A)$ in a patient with a focal ectopic atrial tachycardia arising from the interatrial septum. Electrical activation of the atria is color-coded with early activation displayed in red and late activation displayed in blue/purple as shown in the color bar. Early activation indicates the origin of the focal atrial tachycardia. Radiofrequency energy delivery at the site of early activation at the interatrial septum resulted in elimination of the tachycardia.

The yellow dot denotes the site of the His bundle recording. The position of the tip of the mapping and ablation catheter (MAP) is shown.

Right: Antero-posterior view of a three-dimensional electroanatomic activation map of the left atrium (LA) in a patient with long-standing persistent AF showing the "chaotic" activation pattern of AF. The orange tags denote sites with CFAEs in the coronary sinus. 


\section{Mapping of atrial fibrillation - Future perspectives}

As demonstrated in clinical mapping applications a combination of imaging and mapping techniques provides valuable additional information. While intracardiac ultrasound in combination with mapping and ablation procedures is already used, pilot studies evaluating the use of high resolution ultrasound to scan the epicardial mapping area are ongoing. Once available this technique might provide valuable additional information about the correlation between atrial electrophysiology and underlying tissue structure. It could further be used to define suitable anatomical landmarks for surgical ablation procedures.

The epicardial ablation approach performed during open-heart surgery or in minimally invasive technique is developing rapidly and can be combined with intraoperative high-density contact mapping. The combination of these two techniques provides new opportunities to study the mechanisms underlying $A F$ in patients and probably to individualize the therapeutic approach based on individual mapping parameters. While trials including epicardial contact mapping in patients are ongoing, a safe technique for simultaneous endo-epicardial high resolution mapping in patients is not developed yet.

New perspectives for research and clinical applications will possibly evolve with the noninvasive technique of body surface mapping ${ }^{26}$. This technique acquires 224 body surface electrograms and calculates the epicardial isochrones by integrating the signal morphology and the three dimensional position of each electrode using an individual MRI dataset of the patient studied. While there is already promising data for ventricular arrhythmias the contribution to mapping of $A F$ is still open ${ }^{19,27}$. 
With magnetic and robotic navigation and real-time acquisition of three-dimensional anatomic structures in the electrophysiology laboratory, the accuracy of mapping procedures is increasing further but still lacks the resolution required to display the complex patterns occurring during atrial fibrillation. Although preprocedure imaging with MRI or CT (or periprocedural imaging with rotational angiography (DynaCT) or intracardiac ultrasound and image integration into the acquired maps are the standard of care in many electrophysiology laboratories, the cost-effectiveness of these tools needs to be shown.

New catheter-based technologies are still evaluated as possible options for high resolution endocardial mapping of AF in patients (En Site ${ }^{\circledR}$, St Jude Medical, Inc. Minneapolis, USA) ${ }^{28}$.

With the available mapping technologies from basic and clinical research and their implementation in clinical research we hopefully will be able to address crucial questions on the mechanisms underlying initiation and perpetuation of $A F$ in patients and reach a more profound understanding of the disease in the near future. This should facilitate evaluation and refinement of established and new treatment strategies for AF and increase the efficacy and safety for our patients.

Acknowledgement: The authors would like to thank Sander Verheule for contributing the content of figure 4 . 


\section{References}

1. Allessie MA, Lammers W, Bonke F, Hollen J. Experimental evaluation of Moe's multiple wavelet hypothesis of atrial fibrillation New York: Grune \& Stratton; 1985.

2. Konings KT, Kirchhof CJ, Smeets JR, Wellens HJ, Penn OC, Allessie MA. High-density mapping of electrically induced atrial fibrillation in humans. Circulation. 1994;89(4):1665-1680.

3. Kirchhof C, Chorro F, Scheffer GJ, Brugada J, Konings K, Zetelaki Z, Allessie M. Regional entrainment of atrial fibrillation studied by high-resolution mapping in open-chest dogs. Circulation. 1993;88(2):736-749.

4. Moe GK, Abildskov JA. Atrial fibrillation as a self-sustaining arrhythmia independent of focal discharge. Am Heart J. 1959;58(1):59-70.

5. Konings KT, Smeets JL, Penn OC, Wellens HJ, Allessie MA. Configuration of unipolar atrial electrograms during electrically induced atrial fibrillation in humans. Circulation. 1997;95(5):1231-1241.

6. Nademanee K, McKenzie J, Kosar E, Schwab M, Sunsaneewitayakul B, Vasavakul T, Khunnawat C, Ngarmukos T. A new approach for catheter ablation of atrial fibrillation: mapping of the electrophysiologic substrate. J Am Coll Cardiol. 2004;43(11):2044-2053.

7. Oral H, Chugh A, Good E, Wimmer A, Dey S, Gadeela N, Sankaran S, Crawford T, Sarrazin JF, Kuhne M, Chalfoun N, Wells D, Frederick M, Fortino J, Benloucif-Moore S, Jongnarangsin K, Pelosi F, Jr., Bogun F, Morady F. Radiofrequency catheter ablation of chronic atrial fibrillation guided by complex electrograms. Circulation. 2007;115(20):2606-2612.

8. Mandapati R, Skanes A, Chen J, Berenfeld O, Jalife J. Stable microreentrant sources as a mechanism of atrial fibrillation in the isolated sheep heart. Circulation. 2000;101(2):194-199.

9. Jalife J, Berenfeld $\mathrm{O}$, Mansour M. Mother rotors and fibrillatory conduction: a mechanism of atrial fibrillation. Cardiovasc Res. 2002;54(2):204-216.

10. Kumagai K, Khrestian C, Waldo AL. Simultaneous multisite mapping studies during induced atrial fibrillation in the sterile pericarditis model. Insights into the mechanism of its maintenance. Circulation. 1997;95(2):511-521. 
11. Haissaguerre $M$, Jais $P$, Shah DC, Takahashi A, Hocini M, Quiniou G, Garrigue S, Le Mouroux A, Le Metayer P, Clementy J. Spontaneous initiation of atrial fibrillation by ectopic beats originating in the pulmonary veins. $N \mathrm{Engl}$ J Med. 1998;339(10):659-666.

12. Schuessler RB, Kawamoto $\mathrm{T}$, Hand DE, Mitsuno M, Bromberg BI, Cox JL, Boineau JP. Simultaneous epicardial and endocardial activation sequence mapping in the isolated canine right atrium. Circulation. 1993;88(1):250-263.

13. Wijfels MC, Kirchhof CJ, Dorland R, Allessie MA. Atrial fibrillation begets atrial fibrillation. A study in awake chronically instrumented goats. Circulation. 1995;92(7):1954-1968.

14. Eckstein J, Verheule S, de Groot N, Allessie M, Schotten U. Mechanisms of perpetuation of atrial fibrillation in chronically dilated atria. Prog Biophys $\mathrm{Mol}$ Biol. 2008;97(2-3):435-451.

15. Eckstein J, Maesen B, Verheule S, Allessie M, Schotten U. Endo-Epicardial Dissociation of Electrical Activity Increases During the Development of the Substrate for Atrial Fibrillation in the Goat. Circulation. 2008;118(Suppl):435.

16. Damiano RJ, Jr., Voeller RK. Biatrial lesion sets. J Interv Card Electrophysiol. 2007;20(3):95-99.

17. Damiano RJ, Jr., Schuessler RB, Voeller RK. Surgical treatment of atrial fibrillation: a look into the future. Semin Thorac Cardiovasc Surg. 2007;19(1):39-45.

18. Pruitt JC, Lazzara RR, Ebra G. Minimally invasive surgical ablation of atrial fibrillation: The thoracoscopic box lesion approach. J Interv Card Electrophysiol. 2007;20(3):83-87.

19. Schuessler RB, Damiano RJ, Jr. Patient-specific surgical strategy for atrial fibrillation: promises and challenges. Heart Rhythm. 2007;4(9):1222-1224.

20. Pappone C, Rosanio S, Oreto G, Tocchi M, Gugliotta F, Vicedomini G, Salvati A, Dicandia C, Mazzone P, Santinelli V, Gulletta S, Chierchia S. Circumferential radiofrequency ablation of pulmonary vein ostia: A new anatomic approach for curing atrial fibrillation. Circulation. 2000;102(21):26192628.

21. Oral H, Scharf C, Chugh A, Hall B, Cheung P, Good E, Veerareddy S, Pelosi F, Jr., Morady F. Catheter ablation for paroxysmal atrial fibrillation: segmental pulmonary vein ostial ablation versus left atrial ablation. Circulation. 2003;108(19):2355-2360. 
22. Knecht $S$, Hocini M, Wright M, Lellouche $N$, O'Neill MD, Matsuo S, Nault I, Chauhan VS, Makati KJ, Bevilacqua M, Lim KT, Sacher F, Deplagne A, Derval N, Bordachar P, Jais P, Clementy J, Haissaguerre M. Left atrial linear lesions are required for successful treatment of persistent atrial fibrillation. Eur Heart J. 2008;29(19):2359-2366.

23. Hou Y, Scherlag BJ, Lin J, Zhang Y, Lu Z, Truong K, Patterson E, Lazzara R, Jackman WM, Po SS. Ganglionated plexi modulate extrinsic cardiac autonomic nerve input: effects on sinus rate, atrioventricular conduction, refractoriness, and inducibility of atrial fibrillation. Journal of the American College of Cardiology. 2007;50(1):61-68.

24. Oral H, Chugh A, Yoshida K, Sarrazin JF, Kuhne M, Crawford T, Chalfoun N, Wells D, Boonyapisit W, Veerareddy S, Billakanty S, Wong WS, Good E, Jongnarangsin K, Pelosi F, Jr., Bogun F, Morady F. A randomized assessment of the incremental role of ablation of complex fractionated atrial electrograms after antral pulmonary vein isolation for long-lasting persistent atrial fibrillation. Journal of the American College of Cardiology. 2009;53(9):782-789.

25. Gepstein L, Hayam G, Ben-Haim SA. A novel method for nonfluoroscopic catheter-based electroanatomical mapping of the heart. In vitro and in vivo accuracy results. Circulation. 1997;95(6):1611-1622.

26. Rudy Y. Noninvasive electrocardiographic imaging in humans. $J$ Electrocardiol. 2005;38(4 Suppl):138-139.

27. Wang Y, Cuculich PS, Woodard PK, Lindsay BD, Rudy Y. Focal atrial tachycardia after pulmonary vein isolation: noninvasive mapping with electrocardiographic imaging (ECGI). Heart Rhythm. 2007;4(8):1081-1084.

28. Verma A, Wulffhart Z, Beardsall M, Whaley B, Hill C, Khaykin Y. Spatial and temporal stability of complex fractionated electrograms in patients with persistent atrial fibrillation over longer time periods: relationship to local electrogram cycle length. Heart Rhythm. 2008;5(8):1127-1133. 



\section{Chapter 7}

\section{General Discussion}

Electrophysiology of the epicardial layer and the endocardial bundle network

Breakthrough and ectopic focal discharges

Clinical relevance 
The present study represents an integrative approach to assess mechanisms contributing to the stabilization of AF over time. The focus of this study lies on the 3dimensional nature of $\mathrm{AF}$, the analysis of endo-epicardial differences in electrophysiological properties, transmural conduction and the mechanistic implications this might have.

\section{Electrophysiology of the epicardial layer and the endocardial}

\section{bundle network}

Endo-epicardial differences in electrophysiological properties are a well-studied phenomenon in the ventricular myocardium. Dispersion of refractoriness is known to be arrhythmogenic in ventricular myocardium where it has been shown to be present within the endocardial and the epicardial plane. ${ }^{1,2}$ Distinct differences between the ventricular epicardium and endocardium have been shown to be present for several electrophysiological properties including repolarization time., 4 Antzelevitch described a third region between the epicardial and the endocardial layer (M-Cells) that present electrophysiological and pharmacological properties different from cells in the neighboring layers. ${ }^{5}$ Whether dispersion of refractoriness within the ventricular wall is contributing to the initiation or perpetuation of ventricular arrhythmias is still a matter of debate. ${ }^{6}$ The concordant T-wave observed in the human ECG is a consequence of obvious differences in action potential duration (APD) between the endocardial and the epicardial cells. ${ }^{3}$ This leads to a negative correlation between depolarization and repolarization times with the endocardial cardiomyocytes depolarizing first and having a longer APD. 
Dispersion of atrial epicardial refractory periods has been demonstrated in the goat. Vollmann et al. found an inverse relation of the epicardial refractory period and the distance from the sinus node. These characteristics didn't change when they introduced a 2 week episode of left atrial pacing to change the vector of atrial activation. ${ }^{7}$ To our knowledge no data was available on endo-epicardial differences of refractoriness in the atrium but Wang et al. studied endo- and epicardial actionpotential durations (APD) in superfused atrial tissue of guinea pigs demonstrating a consistently longer APD in the endocardial layer. These pronounced differences greatly diminished during rapid pacing or with premature stimuli that provided evidence that they are a result of $I_{\text {to }}$ current. These findings were further confirmed by selective $I_{\text {to }}$ blockade with 4-aminopyridine that electively prolonged epicardial APDs. ${ }^{8}$

In theory, a pronounced dispersion of refractoriness between neighboring areas in the endocardial or epicardial plane, as well as between corresponding endo- and epicardial sites would favor the existence of reentry and breakthrough events, both leading to an increased stabilization of AF.

In this thesis, we showed that in non-remodeled atria a significant prolongation of endocardial AERP compared to the exactly opposing epicardial site was present. Interestingly, these differences disappeared in the groups with persistent AF, so that the hypothesis of endo-epicardial AERP dispersion as a mechanism contributing to AF stabilization had to be rejected. For all other parameters analyzed (conduction velocity, AFCL, wave size, number of waves, incidence of breakthrough) no significant differences were present between the epicardial layer and the endocardial bundle network at any time. 
Atrial fibrillation leads to structural remodelling with alterations of the atrial wall including myocyte hypertrophy, fibrosis, GAP junction redistribution and neurohumoral changes. ${ }^{9-11}$ Mapping studies in humans and animals showed that in structurally remodelled atria the incidence of conduction block increases and the electropathological substrate of AF becomes more complex. ${ }^{12}$ It has been shown in earlier mapping studies and computer models that as a consequence of altered conduction, electrical dissociation occurs in the two dimensional plane. ${ }^{13,14}$ So far, these studies were limited to either the endocardial or the epicardial surface of the atrial wall. No in vivo high-resolution contact mapping data was available so far on dissociation between the endocardial bundle network and the epicardial layer. Given the complex anatomy of the atrium one would expect dissociation to occur between the epicardial layer and the endocardial bundle network as well (figure 1). In our series of experiments, we demonstrated structural remodelling in the 6 months AF group with myocyte hypertrophy and increased interstitial fibrosis. Within these 6 months of $\mathrm{AF}$, the degree of endo-epicardial electrical dissociation of electrical activity (EED) strongly increased. As electrical remodelling only took place in the first 3 weeks of AF but EED increased further between the 3 weeks and the 6 months AF group structural remodelling is likely to significantly contribute to the occurrence of EED. 


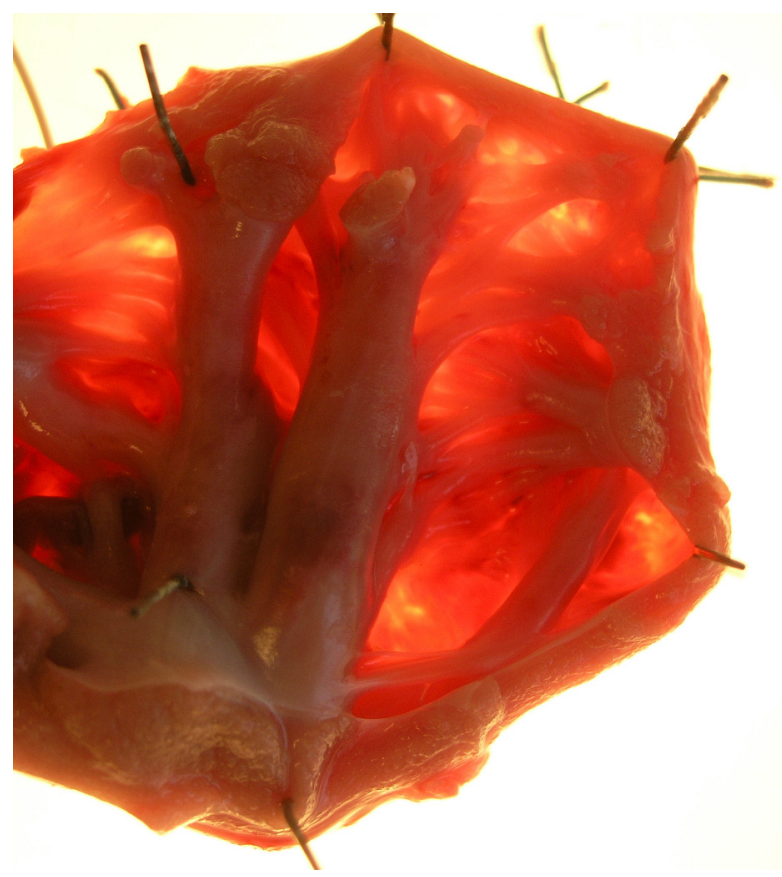

Figure 1: Left atrium of a goat. Thick endocardial trabeculae in the front diverging into thinner muscle bundles and the thin transluminated epicardial layer in the back.

\section{Breakthrough and ectopic focal discharges}

Spontaneous ectopic discharges, predominantly from the pulmonary veins (PV), have been identified as potent triggers of paroxysmal $\mathrm{AF}$ and have been suggested to contribute to the maintenance of the arrhythmia. ${ }^{15,16} \mathrm{PV}$ foci have been widely accepted as triggers of paroxysmal AF and led to the clinical concept of PV isolation. Mechanisms leading to focal activity and/or breakthrough events outside the PVs and their role for the maintenance of AF are still subject of ongoing scientific debate and research.

To identify the origin of these events is of utmost importance because the underlying mechanisms are significantly different. Identification of the mechanism should then lead to a more focused therapeutic approach. 
Atrial fibrillation waves with radial spread of activation were first described as breakthrough events by Konings in patients induced AF. ${ }^{17}$ Origin of these waves could either be ectopic focal discharge triggered by delayed afterdepolarisations or waves originating from the opposing layer of the atrial wall encountering excitable tissue at the site of "breakthrough".

The occurrence and the mechanism underlying ectopic focal discharges during $A F$ is still a matter of debate. ${ }^{3,}{ }^{18-22}$ Hove Madsen reported increased incidences of spontaneous Ca release events ("sparks") in patients with AF. ${ }^{18}$ Sparks can trigger intracellular calcium waves, which then can trigger delayed after-depolarisations and ectopic focal discharge. Knollmann reported on the effect of flecainide on sparks and could demonstrate that flecainide blocks ryanodine receptor $\mathrm{Ca}^{2}$ release channels in the open state and thereby reduces the spark mass. ${ }^{23-25}$ This resulted in reduced after-depolarisations and ectopic action potentials.

Earlier two-dimensional mapping studies in patients with AF analyzed local unipolar electrograms and showed a R-wave morphology at sites of breakthrough which points towards conducted waves rather than local ectopic activity. ${ }^{21}$ Of note, sites of breakthrough were highly variable and almost never repetitive. In the present study we analysed signals from the opposing layer of the atrial wall and did not only determine EED but also tested for the probability of waves in the opposing layer to serve as a source for breakthrough events.

Our analysis revealed that the far majority of breakthrough events could be explained by contralateral activation whereas only a minority of breakthrough fulfilled the criteria for focal ectopic discharge (pairs of two contralateral breakthroughs possibly induced by the same source). 
Interestingly the events of this group were more effectively suppressed by flecainide compared to breakthroughs putatively originating from transmural conduction. This finding supported our hypothesis of two distinct populations of events and represents a good example for the different aspects of an integrative experiment in physiology.

\section{Clinical relevance}

The information that during AF the electrical activity in one layer of the atrial wall is not per se the same as in the opposite layer, is of significant clinical importance. It becomes relevant when standard endocardial mapping is performed with mapping catheters or novel techniques like ECG-Imaging are applied and the opposite site of the atrial wall is not taken into account. ${ }^{26,27}$ ECG-Imaging, for example, only provides information about conduction in the epicardial layer. In case of sinus rhythm or atrial flutter with little or no EED this technique will give reliable information about the atrial electrical activity whereas during $A F$, especially long lasting $A F$, it will provide an incomplete picture. Already since the description of epicardial breakthrough events in patients with induced AF by Konings the missing contralateral (endocardial) information has been an important aspect of the scientific discussion about the origin of theses events. ${ }^{17}$ Indirect evidence pointed towards propagated fibrillation waves from the endocardial layer, and a close positive correlation between AF duration and breakthrough incidence was demonstrated in patients and animal models.

We developed a high resolution in vivo synchronous endo-epicardial mapping technique. 
As a result of this work we concluded

- that endo-epicardial dissociation exists during AF,

- that it increases with AF duration and

- that the majority of breakthrough events most likely originate from transmural conduction.

Nevertheless we postulated that at the same time a significant percentage of breakthrough events derive from ectopic focal discharges and that these events are sensitive to the treatment with flecainide. 


\section{Personal conclusion}

Atrial Fibrillation (AF) is initiated and maintained by a single underlying mechanism in very limited cases only. Far more often multiple underlying mechanisms will contribute to initiation and maintenance of $\mathrm{AF}$.

As a member of a Big Band during my time in Maastricht I thought of a comparison of atrial electrophysiology with an orchestra:

If all musicians play in tune and correct timing, rhythm can easily be maintained by the conductor. If only a single musician plays out of tune or timing this musician needs a very loud and dominant instrument or play in a very vulnerable moment to be able to ruin the whole piece.

But if several members of the orchestra with different instruments play different tempi and disturb the ensemble, it will be hard for the conductor to maintain control and the music will eventually sound chaotic and arrhythmic. Once this happens it can become impossible for the conductor to stop the ensemble and restart it in the appropriate rhythm. Off note to mention our band was well in rhythm most of the time.

$\mathrm{AF}$ is a multifactorial disease with interindividual differences and most often a progression over time.

This makes it such an interesting and challenging subject to study. 


\section{References:}

1. Misier AR, Opthof T, van Hemel NM, Vermeulen JT, de Bakker JM, Defauw JJ, van Capelle FJ, Janse MJ. Dispersion of 'refractoriness' in noninfarcted myocardium of patients with ventricular tachycardia or ventricular fibrillation after myocardial infarction. Circulation. 1995;91(10):2566-2572.

2. Conrath CE, Opthof T. Ventricular repolarization: an overview of (patho)physiology, sympathetic effects and genetic aspects. Prog Biophys Mol Biol. 2006;92(3):269-307.

3. Franz MR, Bargheer K, Rafflenbeul W, Haverich A, Lichtlen PR. Monophasic action potential mapping in human subjects with normal electrocardiograms: direct evidence for the genesis of the T wave. Circulation. 1987;75(2):379386.

4. Antzelevitch C, Sicouri S, Litovsky SH, Lukas A, Krishnan SC, Di Diego JM, Gintant GA, Liu DW. Heterogeneity within the ventricular wall. Electrophysiology and pharmacology of epicardial, endocardial, and $\mathrm{M}$ cells. Circ Res. 1991;69(6):1427-1449.

5. Sicouri S, Antzelevitch C. A subpopulation of cells with unique electrophysiological properties in the deep subepicardium of the canine ventricle. The M cell. Circ Res. 1991;68(6):1729-1741.

6. Chauhan VS, Downar E, Nanthakumar K, Parker JD, Ross HJ, Chan W, Picton P. Increased ventricular repolarization heterogeneity in patients with ventricular arrhythmia vulnerability and cardiomyopathy: a human in vivo study. Am J Physiol Heart Circ Physiol. 2006;290(1):H79-86.

7. Vollmann D, Blaauw $Y$, Neuberger HR, Schotten U, Allessie M. Long-term changes in sequence of atrial activation and refractory periods: no evidence for "atrial memory". Heart Rhythm. 2005;2(2):155-161.

8. Wang ZG, Fermini B, Nattel S. Repolarization differences between guinea pig atrial endocardium and epicardium: evidence for a role of Ito. Am J Physiol. 1991;260(5 Pt 2):H1501-1506.

9. Goette A, Staack T, Rocken C, Arndt M, Geller JC, Huth C, Ansorge S, Klein $\mathrm{HU}$, Lendeckel $\mathrm{U}$. Increased expression of extracellular signal-regulated kinase and angiotensin-converting enzyme in human atria during atrial fibrillation. J Am Coll Cardiol. 2000;35(6):1669-1677.

10. Schotten U, Verheule S, Kirchhof P, Goette A. Pathophysiological mechanisms of atrial fibrillation: a translational appraisal. Physiol Rev. 2011;91(1):265-325. 
11. Ausma J, van der Velden $H M$, Lenders $M H$, van Ankeren EP, Jongsma $H J$, Ramaekers FC, Borgers M, Allessie MA. Reverse structural and gapjunctional remodeling after prolonged atrial fibrillation in the goat. Circulation. 2003;107(15):2051-2058.

12. Allessie MA, de Groot NM, Houben RP, Schotten U, Boersma E, Smeets JL, Crijns HJ. Electropathological substrate of long-standing persistent atrial fibrillation in patients with structural heart disease: longitudinal dissociation. Circ Arrhythm Electrophysiol. 2010;3(6):606-615.

13. Courtemanche $M$, Ramirez RJ, Nattel $\mathrm{S}$. Ionic mechanisms underlying human atrial action potential properties: insights from a mathematical model. $A m \mathrm{~J}$ Physiol. 1998;275(1 Pt 2):H301-321.

14. Kneller J, Ramirez RJ, Chartier D, Courtemanche M, Nattel S. Timedependent transients in an ionically based mathematical model of the canine atrial action potential. Am J Physiol Heart Circ Physiol. 2002;282(4):H14371451.

15. Haissaguerre $M$, Jais $P$, Shah DC, Takahashi A, Hocini M, Quiniou G, Garrigue S, Le Mouroux A, Le Metayer P, Clementy J. Spontaneous initiation of atrial fibrillation by ectopic beats originating in the pulmonary veins. $N$ Engl J Med. 1998;339(10):659-666.

16. Haissaguerre $M$, Hocini $M$, Sanders $P$, Takahashi $Y$, Rotter $M$, Sacher $F$, Rostock T, Hsu LF, Jonsson A, O'Neill MD, Bordachar P, Reuter S, Roudaut $R$, Clementy $J$, Jais $P$. Localized sources maintaining atrial fibrillation organized by prior ablation. Circulation. 2006;113(5):616-625.

17. Konings KT, Kirchhof CJ, Smeets JR, Wellens HJ, Penn OC, Allessie MA. High-density mapping of electrically induced atrial fibrillation in humans. Circulation. 1994;89(4):1665-1680.

18. Hove-Madsen L, Llach A, Bayes-Genis A, Roura S, Rodriguez Font E, Aris A, Cinca J. Atrial fibrillation is associated with increased spontaneous calcium release from the sarcoplasmic reticulum in human atrial myocytes. Circulation. 2004;110(11):1358-1363.

19. Chelu MG, Sarma S, Sood S, Wang S, van Oort RJ, Skapura DG, Li N, Santonastasi M, Muller FU, Schmitz W, Schotten U, Anderson ME, Valderrabano M, Dobrev D, Wehrens $\mathrm{XH}$. Calmodulin kinase II-mediated sarcoplasmic reticulum $\mathrm{Ca} 2+$ leak promotes atrial fibrillation in mice. $J$ Clin Invest. 2009;119(7):1940-1951.

20. Neef S, Dybkova N, Sossalla S, Ort KR, Fluschnik N, Neumann K, Seipelt R, Schondube FA, Hasenfuss G, Maier LS. CaMKII-dependent diastolic SR $\mathrm{Ca} 2+$ leak and elevated diastolic $\mathrm{Ca} 2+$ levels in right atrial myocardium of patients with atrial fibrillation. Circ Res. 2010;106(6):1134-1144. 
21. de Groot NM, Houben RP, Smeets JL, Boersma E, Schotten U, Schalij MJ, Crijns $\mathrm{H}$, Allessie MA. Electropathological substrate of longstanding persistent atrial fibrillation in patients with structural heart disease: epicardial breakthrough. Circulation. 2010;122(17):1674-1682.

22. Greiser $M$, Lederer $W J$, Schotten $U$. Alterations of atrial $\mathrm{Ca}(2+)$ handling as cause and consequence of atrial fibrillation. Cardiovasc Res. 2011;89(4):722733.

23. Hwang HS, Hasdemir C, Laver D, Mehra D, Turhan K, Faggioni M, Yin H, Knollmann BC. Inhibition of Cardiac Ca2+ Release Channels (RyR2) Determines Efficacy of Class I Antiarrhythmic Drugs in Catecholaminergic Polymorphic Ventricular Tachycardia. Circ Arrhythm Electrophysiol. 2011.

24. Hilliard FA, Steele DS, Laver D, Yang Z, Le Marchand SJ, Chopra N, Piston DW, Huke S, Knollmann BC. Flecainide inhibits arrhythmogenic $\mathrm{Ca} 2+$ waves by open state block of ryanodine receptor $\mathrm{Ca} 2+$ release channels and reduction of Ca2+ spark mass. J Mol Cell Cardiol. 2010;48(2):293-301.

25. Watanabe H, Chopra N, Laver D, Hwang HS, Davies SS, Roach DE, Duff HJ, Roden DM, Wilde AA, Knollmann BC. Flecainide prevents catecholaminergic polymorphic ventricular tachycardia in mice and humans. Nat Med. 2009;15(4):380-383.

26. Rudy $Y$. Noninvasive electrocardiographic imaging in humans. $J$ Electrocardiol. 2005;38(4 Suppl):138-139.

27. Wang Y, Cuculich PS, Woodard PK, Lindsay BD, Rudy Y. Focal atrial tachycardia after pulmonary vein isolation: noninvasive mapping with electrocardiographic imaging (ECGI). Heart Rhythm. 2007;4(8):1081-1084. 


\section{Summary}

The present study was conducted to elucidate specific characteristics of endocardial and epicardial atrial activation during atrial fibrillation (AF). We developed a novel tool for synchronous high-density endo-epicardial in-vivo mapping and conducted mapping experiments in goats with acute AF and in goats with 3 weeks or 6 months AF. Aim of the study was to analyze differences between the endocardial and the epicardial atrial activation and possible changes occurring during structural remodeling in long term (persistent) AF. While changes in the early phase of this disease have been analyzed and described in great detail, chronic changes and mechanisms responsible for the increasing stability over time still are analyzed for their impact on the process of AF stabilization. Important mechanisms putatively responsible for the maintenance of AF are discussed in Chapter 2 of this thesis. Among these arrhythmogenic mechanisms are fibrillation waves appearing within the mapping area. In general these waves are referred to as foci or breakthroughs depending on the underlying mechanism suspected. The origin and relevance of these particular fibrillation waves was a major focus of this thesis. In this chapter we introduced the endo-epicardial mapping technique and presented first results.

We could show that endocardial and epicardial electrophysiological characteristics are not identical in normal atria and demonstrate that dissociation of endo-epicardial electrical activity during atrial fibrillation already occurs in normal atria and increases significantly after 3 weeks and 6 months of AF (Chapter 3). This mechanism very likely contributes to the increased stability of AF over time as it increases the surface available for the fibrillation waves to coexist and provides the conditio sine qua non 
for the occurrence of breakthrough events. In this chapter, we developed and validated different algorithms to quantify endo-epicardial dissociation. Independent from the approach used an increase of dissociation after 3 weeks and 6 months was found. In Chapter 4 we developed a wave-based algorithm to quantify endoepicardial dissociation according to the number of dissociated fibrillation waves and the total surface activated out of phase. In this Chapter we focused on the correlation between endo-epicardial dissociation and the possible transmural origin of breakthrough events. We could demonstrate for the first time contralateral activation maps at the site of breakthrough and test the hypothesis that breakthrough events are a result of transmural conduction originating from contralateral activation appearing at the site of breakthrough. We identified transmural conduction as the leading mechanism of breakthrough leaving only a minor fraction of events unlikely to result from contralateral activity. We concluded that the events not explainable by transmural conduction are more likely to originate from ectopic focal discharge or concealed intramural bundles. Projects to further test this hypothesis are ongoing and will complement our data in the near future.

Class $1 \mathrm{C}$ antiarrhythmic Drugs are recommended for cardioversion of AF in the present guidelines for the treatment of AF. Although extensively studied over the past years the exact mechanism how these drugs cardiovert are not known. Some effects, for example slowing of conduction, should even have a proarrhythmic rather than an antiarrhythmic effect. Attracted by this open question we tested the class IC compound flecainide in our goat model in Chapter $\mathbf{5}$ and found that flecainide significantly reduces endo-epicardial dissociation. It even more significantly reduces the incidence of breakthrough events. Interestingly, we could demonstrate that the 
effect of flecainide on breakthrough events putatively originating from focal ectopic discharge is more pronounced than the effect on breakthroughs putatively originating from contralateral activity. The suppression of breakthrough and reduction of endoepicardial dissociation are likely to contribute to the antiarrhythmic properties of this compound.

To further understand the changes occurring in the atrial wall during persistent AF, more mapping studies in patients are conducted at present. While standard clinical mapping techniques are designed to provide "snapshots" of the electric activity correlated with the individual anatomy of the patient, our ongoing studies implement high-density contact mapping in surgical patients. The different mapping techniques are reviewed in Chapter 6. 


\section{Samenvatting}

De studie die wordt voorgesteld in dit proefschrift is uitgevoerd om meer inzicht te krijgen in de specifieke eigenschappen van de elektrische activiteit tijdens atriumfibrilleren (AF) aan de endocardiale en de epicardiale zijde van de atriale wand. Voor dit doel hebben we een nieuw meetinstrument ontworpen, namelijk een elektrode die ons toeliet om met hoge resolutie en tegelijkertijd endo- en epicardiaal de elektrische activatie te mappen in vivo. Vervolgens hebben we deze nieuwe elektrode gebruikt om mapping experimenten uit te voeren in geiten met acuut AF en in geiten die reeds gedurende 3 weken en gedurende 6 maanden in AF waren. Het doel van dit proefschrift was tweevoudig: vooreerst het bestuderen van de verschillen tussen het endocardiale en het epicardiale activatiepatroon. Daarnaast het opsporen van mogelijke verandering tijdens de structurele hermodellering in langdurig bestaand AF (persistent AF). Hoewel de veranderingen die plaatsvinden

tijdens de vroege fase van de ziekte reeds uitgebreid bestudeerd en in detail beschreven zijn, is dat niet het geval voor de langetermijn (chronische) veranderingen. Immers, de impact van chronische veranderingen en mechanismen op het stabiliseren van AF door de tijd heen wordt nog steeds in kaart gebracht. In Hoofdstuk 2 van dit proefschrift worden belangrijke mechanismen besproken die vermoedelijk verantwoordelijk zijn voor de instandhouding van AF. Een voorbeeld van deze aritmogene mechanismen zijn de golven die opduiken in het meetgebied. Over het algemeen worden deze golven benoemd als 'focale' golven of als 'doorbraak' golven, afhankelijk van het veronderstelde onderliggende mechanisme. Zowel de oorsprong als de betekenis van deze aparte golven was een belangrijk 
aandachtspunt in dit proefschrift. In dit eerste hoofdstuk worden de techniek en de eerste resultaten van het endo-epicardiaal mappen besproken.

We hebben kunnen aantonen dat in normale voorkamers de elektrofysiologische eigenschappen aan de endocardiale en de epicardiale zijde van de atriale wand niet gelijk zijn. Tevens laten we zien dat er reeds in normale voorkamers dissociatie optreedt van de endo-epicardiale elektrische activiteit en dat deze dissociatie significant toeneemt na 3 weken en 6 maanden van AF (Hoofdstuk 3). Het is zeer waarschijnlijk dat deze dissociatie bijdraagt aan het toenemen van de stabiliteit van AF met de tijd. Immers, het verhoogt de oppervlakte die golven toelaat om naast elkaar te bestaan en het vormt een conditio sine qua non voor het optreden van transmurale geleiding in de vorm van 'doorbraak' golven. In het tweede hoofdstuk hebben we algoritmen ontwikkeld en gevalideerd om deze endo-epicardiale dissociatie te kwantificeren. We zagen dat, onafhankelijk van welke benadering we gebruikten, de dissociatie toenam na 3 weken en 6 maanden van AF. In Hoofdstuk 4 ontwikkelden we een algoritme om de dissociatie te kwantificeren op basis van het aantal gedissocieerde fibrillatiegolven en het totale oppervlak van deze golven dat uit fase werd geactiveerd. Dit hoofdstuk legt zich toe op de verhouding tussen endoepicardiale dissociatie en de mogelijk transmurale oorsprong van deze golven. We zijn erin geslaagd om voor de eerste keer de contralaterale activatie kaarten te tonen op de plaats waar 'doorbraak' van geleiding plaats vindt. Zo was het mogelijk de hypothese te toetsen of het 'doorbreken' van de geleiding effectief het gevolg is van transmurale geleiding met oorsprong in het gebied aan de andere zijde van de plaats van 'doorbraak'. Onze studie identificeerde transmurale geleiding als hoofdmechanisme in het optreden van 'doorbraak' van geleiding. Enkel in een klein 
aantal van dergelijke 'doorbraken' leek het onwaarschijnlijk dat deze het gevolg waren van transmurale geleiding. We kwamen dan ook tot de conclusie dat dergelijke doorbraken die niet verklaarbaar zijn door contralaterale activatie hoogstwaarschijnlijk het gevolg zijn van ectope focale ontlading of geleiding in verdoken intramurale spierbundels. Heden lopen er projecten die deze hypothese verder toetsen. In de nabije toekomst zullen de resultaten hiervan onze stellingen verder onderbouwen.

In de huidige richtlijnen voor de behandeling van AF worden Klasse $1 \mathrm{C}$ anti-aritmica aanbevolen voor de cardioversie van AF. Ondanks het feit dat deze medicijnen de laatste jaren erg goed bestudeerd zijn, is het onbekend via welk mechanisme ze in staat zijn om AF te cardioverteren. Sommige effecten, zoals bijvoorbeeld het vertragen van de geleiding, zouden eerder pro-aritmisch dan anti-aritmisch moeten werken. Geïntrigeerd door deze onbeantwoorde vraag, hebben we flecainide, een klasse $1 \mathrm{C}$ anti-aritmicum, getest in ons geitenmodel. In Hoofdstuk $\mathbf{5}$ tonen we aan dat flecainide endo-epicardiale dissociatie significant doet afnemen en dat het aantal 'doorbraken' van geleiding er nog meer (significant) door vermindert. Het is interessant om te weten dat het effect van flecainide meer invloed heeft op ‘doorbraken' die vermoedelijk het gevolg zijn van focale ectope ontladingen dan op diegene die vermoedelijk het gevolg zijn van transmurale geleiding. Het onderdrukken van deze 'doorbraken' en het verminderen van endo-epicardiale dissociatie draagt vermoedelijk ook bij aan de anti-aritmische werking van deze stof. Om de veranderingen die optreden in de atriale wand tijdens persistent AF beter te begrijpen, worden nu ook in patiënten mapping studies uitgevoerd. De technieken die standaard in de kliniek worden gebruikt zijn ontwikkeld om momentopnames van 
elektrische aktiviteit te nemen, gecorreleerd aan de individuele anatomie van een patiënt. Onze studies daarentegen betreffen mapping studies met hoge resolutie in chirurgische patiënten. De verschillende technieken die gebruikt worden om te mappen zijn beschreven in Hoofdstuk 6. 



\section{Acknowledgements / Nawoord}

\section{Weet je wat wij zouden moeten doen....? (You know what we should do..?)}

This is very likely the most frequent question I heard from Uli Schotten.

His numerous ideas and the incomparable scientific environment at the Cardiovascular Research Institute Maastricht provide the base for independent and quality orientated research (figure 1). I would like to thank you Uli for giving me the opportunity to become a member of this group, for your continuous support of my research as my mentor and promotor, and I very much value the personal friendship that developed between our families in the past years.

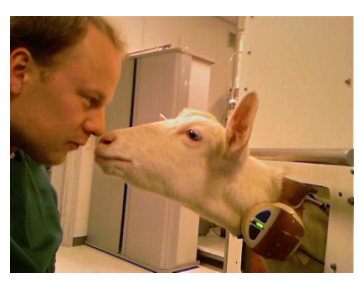

Figure 1: Research

When I first met Maurits Allessie he was just coming back from France, sparkling with energy and ideas. I was asked to present my research plans on a "working meeting" which of course I did. Retrospectively the Nawoord of Ulis Thesis should have been the first manuscript to read before starting research in Maastricht as it contains some important information on the natural courses of working meetings of our group. ${ }^{1}$ In conclusion, the only component that could be reliably predicted was an extended and unique scientific discussion sometimes challenging my own physiological limits. But what do you expect from a Physiology department? 
I certainly would like to thank you too, Maurits for being my promoter and supporting my thesis and scientific career.

Without any doubt this work profits from local, national and international networks and it is always inspiring to exchange ideas with colleagues of Physiology Department, the Cardiology Department of the AZM, the Department of Physiology in Amsterdam, the Leducq network and the AFNET. Still it only thrives, grows and eventually becomes a success thanks to colleagues and friends who provide support for the everyday challenges. Our lab technicians Arne van Hunnik, Marion Kuiper, Anniek Lampert, Jan Hollen and the people from CPV took care of the animals, performed demanding surgery and developed solutions for literally every problem. Without their skills we would not have been able to perform these experiments.

The same accounts for Stef Zeemering who entered the stage late but almost momentarily presented never-ending updates of custom programmed analysis software that hasn't crashed a single time!

In about the same time period Bart Maesen claimed a chair in our office and it was a joy to work with him every since. It is as much for professional as for personal reasons that I dearly hope we will manage to continue our scientific cooperation and friendship in the time to come. Dominik Linz joined our team shortly after my leave and instantly provided great input.

I would like to express very special thanks for all the help and advice given by Sander Verheule who impressed and surprised me in many different ways. After all I can't recall a thing I asked him couldn't answer (and I asked various things). He provided extensive input to our project and this manuscript and last but not least introduced not only me to the Apple-world. 
This list would not be complete without Maura Greiser. We were colleagues for my entire time in Maastricht, became friends and who knows, if I would have stayed longer, I probably even would have become a "Channel"- person too (not the fashion brand though) just because of the enthusiasm she expresses when she talks about a Calcium wave.

Along with all the professional aspects of my work in Maastricht I very much enjoyed our rich social life here. Starting in our department with "Vlaai in de koffiekamer", the honor to be "De bier-Commissaris" and member of the "Dagje Uit Commissie" (only to name a few), our everyday life with our dear warmhearted neighbors in St. Pieter, the dutch-german $\mathrm{BBQ}$ with our dutch and international friends and the SLIM Kumulus Big Band of Mark Huynen. They are all still part of our lives.

Having moved back to my former workplace in Basel too I would like to take this opportunity to thank Dietrich Kalusche who was the person to raise my interest in electrophysiology, Andre Perruchoud who gave me an example of how to be a dedicated coach and team-leader and the teams of the Cardiology Department and the Department of Medicine at the University hospital in Basel (figure 2).

Figure 2: Clinic

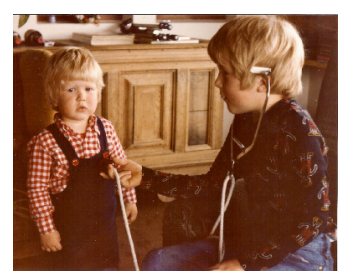

Finalizing this thesis I once more appreciated the skills of our neighbor and friend Michael Bögle who solved all graphics problems with a smile on his face. 
Having mentioned so many people, and surely having forgotten as many (for which I apologize), I "saved the best for last".

I would like to thank my mother Erika and my brothers Carsten and Philipp (figure 2) for their support.

Even though it does not at all compensate for the time I was physically and/or mentally not present I would like to dedicate this thesis to my beloved wife Sandra, my sons Paul and Max and my daughter Marie.

You are the music in my life.

Weet je Uli, wat ik heel graag zou willen doen....?

Contact houden met elkaar en onze samenwerking voortzetten.

\section{Reference:}

1. Schotten U. Mechanisms of Atrial Paralysis due to AtrialFibrillation - An Integrative Study in Man and Goat. Maastricht: Fysiology, Maastricht University; 2003. 


\section{Curriculum vitae}

\section{Jens Eckstein, MD}

Born May 7th 1969 in Heilbronn a.N.,

Married to Sandra Eckstein, 3 children (2004, 2006 \& 2010)

Present

Staff member at University Hospital Basel,

Department of Medicine

$06 / 2010$

Board Certification Cardiology

$07 / 2009-11 / 2010$

Fellowship, University Hospital Basel,

Department of Cardiology

$04 / 2005-03 / 2010$

Research Fellowship, Cardiovascular Research

Institute Maastricht, Maastricht University,

Dept. of Physiology

03/2005

Board Certification Internal Medicine

$01 / 2005-03 / 2005$

Scientific Committee medArt Basel

$10 / 2003-12 / 2004$

Fellowship, University Hospital Basel,

Department of Cardiology

$10 / 2001-09 / 2003$

Fellowship, University Hospital Basel,

Department of Medicine

$08 / 1998-09 / 2001$

Fellowship, Herzzentrum Bad Krozingen,

Department of Electrophysiology 
MD, University of Lübeck,

Thesis: "Development and in vitro testing of an artificial heart valve built from venous valves for pulmonary valve replacement"

06/1998

Medical Degree, University of Lübeck

$1991-1998$

Internship and medical training,

University of Freiburg,

University of Lübeck,

Tulane University New Orleans

University Hospital Basel

$1988-1990$

Civil service, Paramedics Degree

$1979-1988$

High school Diploma,

Zabergäu Gymnasium Brackenheim 


\section{Grants \& Awards}

EU Marie Curie, Early career research grant, Cardiovascular Research Institute Maastricht, Maastricht University development Grant 



\section{Publications}

\section{Articles}

1. Kirchhof P, Marijon E, Fabritz L, Li N, Wang W, Schulte K, Hanstein J, Schulte JS, Vogel M, Mougenot N, Laakmann S, Fortmueller L, Eckstein J, Verheule S, Kaese S, Staab C, Grote-Wessels S, Schotten U, Moubarak H, Wehrens XH, Schmitz W, Hatem S, Muller F. Overexpression of cAMP-response element modulator causes abnormal growth and development of the atrial myocardium resulting in a substrate for sustained atrial fibrillation in mice. Int J Cardiol. 2011; in press.

2. Eckstein J, Maesen B, Linz D, Zeemering S, van Hunnik A, Verheule S, Allessie M, Schotten U. Time Course and Mechanisms of Endoepicardial Electrical Dissociation during Atrial Fibrillation in the Goat. Cardiovasc Res 89, 816-24.

3. Blana A, Kaese S, Fortmuller L, Laakmann S, Damke D, Bragt KV, Eckstein J, Piccini I, Kirchhefer U, Nattel S, Breithardt G, Carmeliet P, Carmeliet E, Schotten U, Verheule S, Kirchhof P, Fabritz L. Knock-in gain-of-function sodium channel mutation prolongs atrial action potentials and alters atrial vulnerability. Heart Rhythm 7, 1862-9.

4. Kuiper HRM, Eckstein J. De Bunny Snuggle. Biotechniek. 2010;49(4):157-159.

5. Eckstein J, Kuehne M, Osswald S, Schotten U. Mapping of atrial fibrillation--basic research and clinical applications. Swiss Med Wkly. 2009;139(35-36):496-504.

6. Eckstein J, Verheule S, de Groot N, Allessie M, Schotten U. Mechanisms of perpetuation of atrial fibrillation in chronically dilated atria. Prog Biophys Mol Biol. 2008;97(2-3):435-451.

7. Eckstein J, Koller MT, Zabel M, Kalusche D, Schaer BA, Osswald S, Sticherling C. Necessity for surgical revision of defibrillator leads implanted long-term: causes and management. Circulation. 2008;117(21):2727-2733.

8. Eckstein J, Savic S, Eugster T, Pargger H, Gurke L, Pechula M, Steiger J, Mayr M. Extensive calcifications induced by hyperphosphataemia caused by phosphate-based enema in a patient after kidney transplantation. Nephrol Dial Transplant. 2006;21(7):2013-2016.

9. Eckstein J, Rueter F, Linka A. Images in cardiology. Acute dyspnoea two days after chest pain. Heart. 2005;91(11):1468. 
10. Ammann $P$, Sticherling $C$, Kalusche D, Eckstein J, Bernheim A, Schaer $B$, Osswald S. An electrocardiogram-based algorithm to detect loss of left ventricular capture during cardiac resynchronization therapy. Ann Intern Med. 2005;142(12 Pt 1):968-973.

11. Bernheim A, Ammann P, Sticherling C, Burger P, Schaer B, BrunnerLa Rocca HP, Eckstein J, Kiencke S, Kaiser C, Linka A, Buser P, Pfisterer M, Osswald S. Right atrial pacing impairs cardiac function during resynchronization therapy: acute effects of DDD pacing compared to VDD pacing. J Am Coll Cardiol. 2005;45(9):1482-1487.

12. Giambarba C, Eckstein J. T-Negativierungen und Verlängerung der QTc-Zeit nach wiederholten epileptischen Anfällen. Schweiz Med Forum. 2003;3:323-325.

\section{Abstracts}

Kaese S, Kirchhof P, Fabritz L, Vogel M, Laakmann S, Schulte JS, Eckstein J, Staab A, Grote-Wessels S, Allessie M, Breithardt G, Schotten U, Hatem S, Marijon E, Moubarak G, Schmitz W, Mueller FU. Triggered activity linked to sarcoplasmic reticulum calcium release and electrical isolation of cardiomyocytes cause atrial arrhythmias in a transgenic model with increased expression of CREM. Clin Res Cardiol. 2009.

Eckstein J, Maesen B, Zeemering S, Verheule S, Allessie M, Schotten U. Spatial Distribution of Endo- Epicardial Dissociation during Atrial Fibrillation in the Goat Model of AF. Clin Res Cardiol. 2009.

Eckstein J, Maesen B, Verheule S, Allessie M, Schotten U. GAP134 does not significantly influence Atrial Fibrillation (AF) Cycle Length (CL), Cardioversion Rate, Conduction Velocity and RR-Interval in Short-term and Long-term AF in the Goat Model of AF. Clin Res Cardiol. 2009.

Eckstein J, Verheule S, Hunnik van A, Kuiper M, Allessie M, Schotten U. Endo- epicardial Dispersion of Refractoriness does not Contribute to Stabilization of AF over Time in a Goat Model of AF. Clin Res Cardiol. 2008;97(Suppl 1).

Eckstein J, Verheule S, Hunnik van A, Kuiper M, Allessie M, Schotten U. Atrial Fibrillation Enhances Endo-epicardial Electrical Dissociation Independently from Shortening of AF Cycle Length. Clin Res Cardiol. 2008;97(Suppl 1).

Eckstein J, Maesen B, Verheule S, Allessie M, Schotten U. Endo-Epicardial Dissociation of Electrical Activity Increases During the Development of the Substrate for Atrial Fibrillation in the Goat. Circulation. 2008;118 (Suppl):S_435. 
Eckstein J, Verheule S, Allessie M, Schotten U. Flecainide Resynchronizes Endo-epicardial Electrical Dissociation in a Goat Model of AF. Clin Res Cardiol. 2008;97(Suppl 1).

Fabritz L, Kirchhof P, Vogel M, Laakmann S, Eckstein J, Schotten U, GroteWessels S, Allessie M, Schmitz W, Breithardt G, Mueller FU.

Elektrophysiologische und strukturelle Veraenderungen, die Vorhofflimmern vorausgehen, untersucht in einem transgenen Modell spontanen Vorhofflimmerns (CREM-transgene Maus). Clin Res Cardiol. 2007;96(Suppl 1).

Eckstein J, Verheule S, Allessie M, Schotten U. Endo- epicardial Dispersion of Refractoriness in the Goat model of AF. Circulation. 2007;116(Suppl):II_279II_280.

Eckstein J, Coppini R, Verheule S, Allessie M, Schotten U. Three-dimensional Organization of Atrial Fibrillation: First Insights from Simultaneous Epienodcardial High Density Mapping in Goats. Heart Rhythm. 2007;4(Suppl 5):175.

Eckstein J, Coppini R, Verheule S, Allessie M, Schotten U. Dreidimensionale Organisation von Vorhofflimmern: Erste Einblicke in Simultanes Endoepicardiales Hochaufloesendes Mapping in Ziegen. Clin Res Cardiol. 2007;96(Suppl 1).

Eckstein J, Zabel M, Kalusche D, Ammann P, Schaer B, Osswald S, Sticherling C. Management of Lead Failure in Patients with Implantable Cardioverter Defibrillators (ICD). Heart Rhythm. 2006(Suppl).

Arranto C, Eckstein J, Bodmer H, Hunziker P, Eriksson U. Detection of heartspecific autoantibodies after myocardial infarction using a novel porcine alpha-myosin based ELISA. Swiss Archives of Neurology and Psychiatry. 2005;156(Suppl):209.

Sticherling C, Ammann P, Kalusche D, Eckstein J, Bernheim A, Schaer B, Osswald S. Neuartiger EKG-Algorithmus zur Detektion der linksventrikulären Stimulation bei kardialer Resynchronisationstherapie. Z Kardiol. 2005;94(Suppl 1).

Eckstein J, Zabel M, Kalusche D, Ammann P, Schaer B, Osswald S, Sticherling C. Management of Lead Failure in Patients with Implantable Cardioverter Defibrillators (ICD). Z Kardiol. 2005;94(Suppl 1).

Eckstein J, Schaer B, Sticherling C, Ammann P, Cron T, Osswald S. Clinical impact of post implant shock-testing in patients with a newly implanted ICD,. Kardiovaskulaere Medizin. 2004;7(Suppl 6):42. 
Eckstein J, Zabel M, Kalusche D, Ammann P, Schaer B, Osswald S, Sticherling C. Management of Lead Failure in Patients with Implantable Cardioverter Defibrillators (ICD). Kardiovaskulaere Medizin 2004;7(Suppl 6):41.

Ammann P, Eckstein J, Bernheim A, Schaer B, Sticherling C, Osswald S. A novel ECG-algorithm to detect loss of left ventricular capture in patients with resynchronisation therapy (CRT). Kardiovaskulaere Medizin. 2004;7(Suppl 6):30.

Bernheim A, Ammann P, Burger P, Schaer B, Sticherling C, Eckstein J, Linka A, Buser P, Pfisterer M, Osswald S. Cardiac resynchronisation therapy: VDDor DDD-mode? How much do we lose by right atrial pacing? Kardiovaskulaere Medizin. 2004;7(Suppl 6).

Schaer B, Ammann P, Bernheim A, Eckstein J, Sticherling C, Cron T, Osswald S. Should cardiac resynchronisation therapy always be combined with an ICD? Kardiovaskulaere Medizin 2004;7(Suppl 6).

Ammann P, Bernheim A, Schaer B, Eckstein J, Sticherling C, Osswald S. Improvment of left ventricular function by cardiac resynchronisation therapy: electromechanical remodeling or pacing only?,. Kardiovaskulaere Medizin. 2004;7(Suppl 6):41.

Guldner NW, Eckstein J, Pohl M, Wendt MO, Sievers HH. The avs valve: a new experimental autologous heart valve constructed from valve bearing segments of femoral veins. Int. J. Artificial Organs. 2003;28(7):609.

Eckstein J, Mayr M, Eugster T, Savic S, Steiger J. Harmful use of phosphatebased enema after kidney transplantation. Swiss Medical Weekly. 2002;132(Suppl 133):22.

Eckstein J, Weirich J, Kalusche D, Susilo R. Sotalolinduced QTc-prolongation can be prevented by potassium/magnesium supplementation. European Heart Journal. 2001;22(Suppl):56.

Guldner NW, Eckstein J, Pohl M, Wendt MO, Sievers HH. Development and in vitro-tests of a new heart valve from autologous vein segments (AVS valve). The Thoracic and Cardiovascular Surgeon. 2000(Suppl).

Guldner NW, Klapproth P, Eckstein J, Gauer IC, Pohl M, Wendt MO, Sievers $\mathrm{HH}$. AVS valve: Development and in vitro-tests of a new heart valve from autologous vein segments. J Heart Valve Disease. 1999(Suppl). 